Portland State University

PDXScholar

Spring 6-6-2017

\title{
Primary Care and Mental Health Integration in Coordinated Care Organizations
}

Robin Lynn Baker

Portland State University

Follow this and additional works at: https://pdxscholar.library.pdx.edu/open_access_etds

Part of the Medicine and Health Sciences Commons

Let us know how access to this document benefits you.

\section{Recommended Citation}

Baker, Robin Lynn, "Primary Care and Mental Health Integration in Coordinated Care Organizations" (2017). Dissertations and Theses. Paper 3616.

https://doi.org/10.15760/etd.5508

This Dissertation is brought to you for free and open access. It has been accepted for inclusion in Dissertations and Theses by an authorized administrator of PDXScholar. Please contact us if we can make this document more accessible: pdxscholar@pdx.edu. 
Primary Care and Mental Health Integration in Coordinated Care Organizations

by

Robin Lynn Baker

A dissertation submitted in partial fulfillment of the requirements for the degree of

Doctor of Philosophy

in

Health Systems and Policy

Dissertation Committee:

Neal Wallace, Chair

Sherril Gelmon

Traci Rieckmann

Matthew Carlson

Portland State University

2017 
@ 2017 Robin Lynn Baker 


\section{Abstract}

The prevalence of untreated and undertreated mental health concerns and the comorbidity of chronic conditions and mental illness has led to greater calls for the integration of primary care and mental health. In 2012, the Oregon Health Authority authorized 16 Coordinated Care Organizations (CCO) to partner with their local communities to better coordinate physical, behavioral, and dental health care for Medicaid recipients. One part of this larger effort to increase coordination is the integration of primary care and mental health services in both primary care and community mental health settings.

The underlying assumption of $\mathrm{CCO}$ is that organizations have the capacity to fundamentally change how health care is organized, delivered, and financed in ways that lead to improved access, quality of care, and health outcomes. Using the Rainbow Model of Integrated Care (RMIC), this study examined the factors that impact organizational efforts to facilitate the integration of primary care and mental health through interviews with executive and senior staff from three CCOs. The RMIC focuses attention on the different levels at which integration processes may occur as well as acknowledges the role that both functional and normative enablers of integration can play in facilitating integration processes within as well as across levels. The following research question was explored: What key factors in Oregon's health care system impede or facilitate the ability of Coordinated Care Organizations to encourage the integration of primary care and mental health? 
Using a case study approach, this study drew upon qualitative methods to examine and identify the factors throughout the system, organizational, professional, and clinic levels that support CCO efforts to facilitate the integration of primary care and mental health. Fourteen primary interviews were conducted with executive and senior staff. In addition, eleven secondary interviews from a NIDA funded project as well as twenty-four key CCO documents from three CCOs were also included in this study.

The RMIC was successful in differentiating extent of CCO integration of primary care and mental health. Findings demonstrate that normative and functional enablers of integration were most prevalent at the system and organization level for integrating mental health into primary care for these three CCOs. However, there was variation in CCO involvement in the development of functional and normative enablers of integration at the professional and clinic levels. Normative and functional enablers of integration were limited at all of the RMIC levels for integrating primary care into community mental health settings across all three CCOs.

The Patient-Centered Primary Care Home model provided CCOs with an opportunity to develop functional and normative enablers of integration for integrating mental health in primary care settings. The lack of a fully developed model for integrating primary care services in community mental health settings serves as a barrier for reverse integration. An additional barrier is the instability of community mental health as compared to primary care; contributing factors 
include historically low wages and increased administrative burden. System wide conversations about where people are best served (i.e., primary care or community mental health) has yet to occur; yet these conversations may be critical for facilitating cross-collaboration and referral processes. Finally, work is needed to create and validate measures of integration for both primary care and community mental health settings. Overall findings confirm that integrating primary care and mental health is complex but that organizations can play an important role by ensuring the development of normative and functional enablers of integration at all levels of the system. 


\section{Dedication}

To my daughter Aria Jae Pearce

Whatever you want to achieve in life, believe in yourself and set out to do it. Never sell yourself short. Never compare yourself to others. Remember that you are resilient and strong, but there is no shame in reaching out for help when you need it. And above all else, don't forget that whoever said we were born perfect lied. We were born imperfect and we are beautiful for it.

To Barry Mac Kantner

I miss you every day. I hope that you finally found peace in the great beyond. 


\section{Acknowledgements}

I entered this program with trepidation. As someone who struggles with anxiety and is riddled with self-doubt, it felt like an act of hubris to enter a PhD program. The first year in the program I was convinced that someone would eventually figure out that I didn't belong and kindly show me the exit. Along the way, life happened and sometimes it was unkind, but I have been blessed with mentors, friends, and family who helped when they could and simply listened when they couldn't. None of this would have been possible without them.

First, I would like to extend my gratitude to my committee members, who guided me throughout the entire process. To my chair, Neal Wallace, I remember the first day I awkwardly asked you to be my advisor. I remember the comfort you offered when my family faced an emergency. I also remember when you told me you could only be my chair if I did quantitative research. Thanks for agreeing to be my chair even though I did a qualitative study. I was incredibly fortunate to have you for my chair and now that I am at the end, I know with a certainty that your sense of humor and steady support have been instrumental in my ability to persevere.

My sincere appreciation also goes Sherril Gelmon. Sherril, you pushed me outside my comfort zone many times over the years and forced me to realize that I am far more capable than I believed. Thank you for believing in me even when I didn't believe in myself. Finally, thank you to Traci Rieckmann and Matthew Carlson for giving me your time and feedback. 
To my friends, Cady, Lesley, Amancia, and the entire Burdsall family, there are no words for the amount of help you provided me and my family over the years. I would not be here today without you. Thank you for having confidence in me and cheering me on along the way. Thank you to Joanne for the years of encouragement even though we've never met in real life; one day, we're going to get to sit down and enjoy a cup of coffee together. To Briana, Will, Marissa, and Shaun - your support and friendship were instrumental to my survival near the end.

To my family, thank you for believing in me. Pops, we may not always see eye to eye but I love you and I always will. Mum, I admire your strength and courage. I have no idea what it must have been like to come to a new country and learn a new language. To my sister and my sweet little niece Evie, never forget that I love you both dearly and will always be here for you. To Phillip, thank you for providing for our small family with hard work and the occasional nurturing meal. Many thanks for ensuring a stock of energy drinks for the many allnighters I pulled over the years. And to my wonderfully unique and quirky Aria Jae, thank you for listening to my practice lectures and putting up with a cranky mom at times over the years.

Last but not least, thank you to Collin Fellows. None of this would be possible without you. Over 11 years ago, you told me that I was bright enough to apply for the Master's Program in Sociology and so I did. Small, seemingly insignificant actions can change lives. 


\section{Table of Contents}

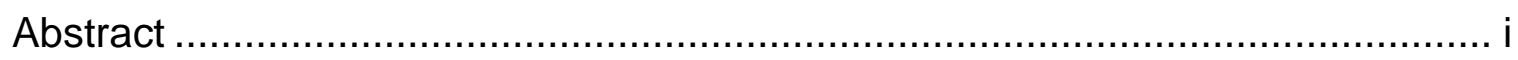

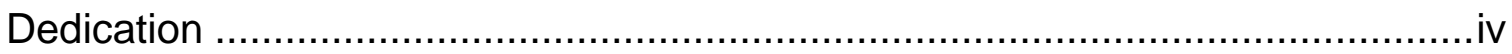

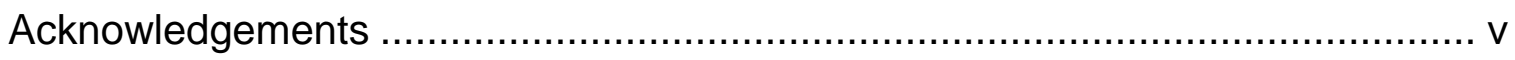

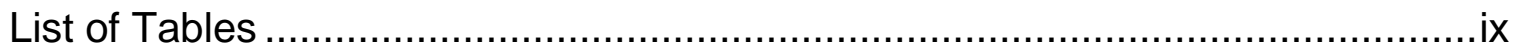

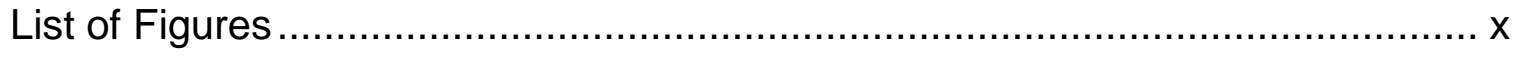

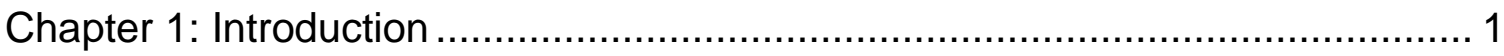

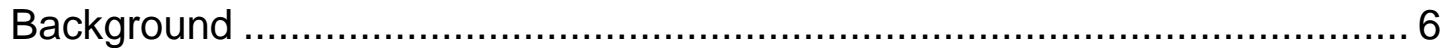

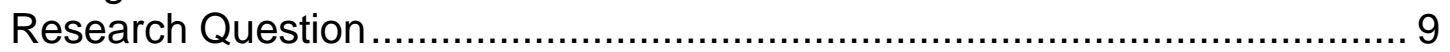

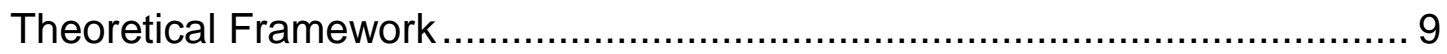

Conceptual Framework .................................................................. 11

Statement of Problem ................................................................... 13

Purpose and Significance of the Study ………................................... 15

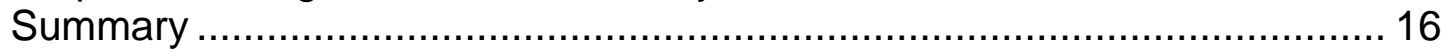

Chapter 2: Review of the Literature ............................................................. 18

Chapter Organization .................................................................... 18

The Case for Primary Care and Mental Health Integration .......................... 19

Larger National Context................................................................... 24

Oregon and Health System Transformation ............................................ 39

Clarifying Primary Care and Mental Health Integration............................... 46

Evidence for Primary Care and Mental Health Integration........................... 54

Barriers to Primary Care and Mental Health Integration ............................. 57

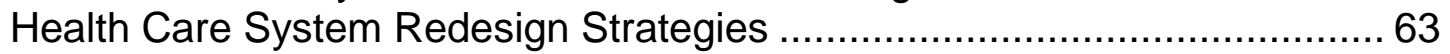

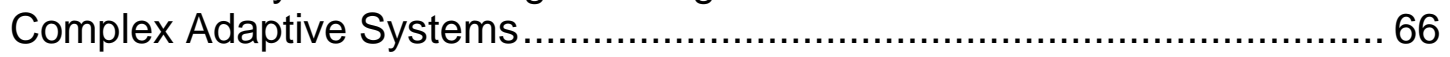

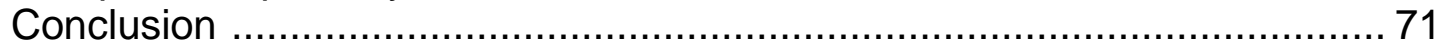

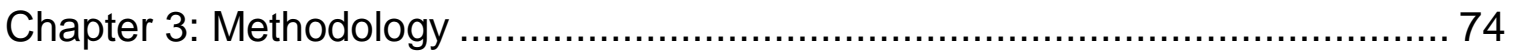

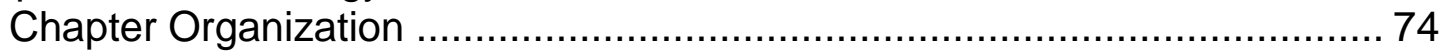

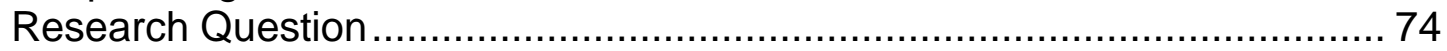

Research Design Overview and Rationale ........................................... 75

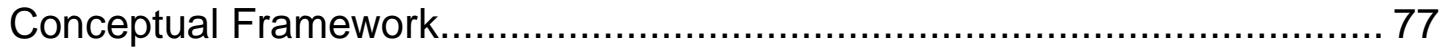

Selection of Coordinated Care Organizations............................................ 80

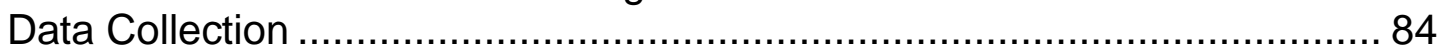

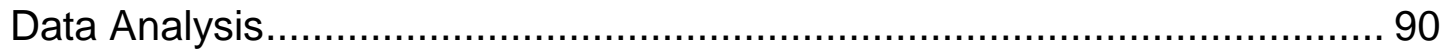

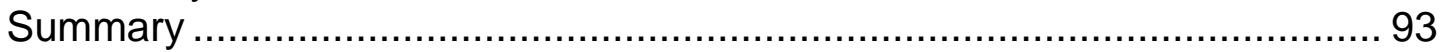


Chapter 4: Presentation and Analysis of Data ................................................. 95

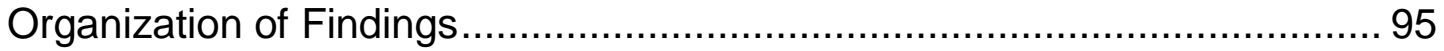

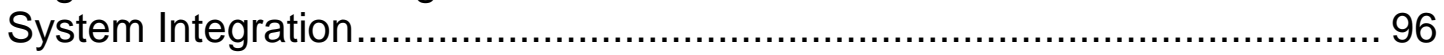

CCO Case Specific Findings ........................................................... 109

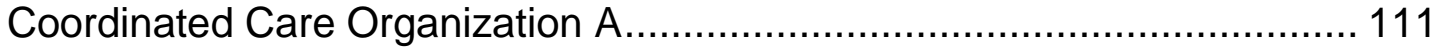

Coordinated Care Organization B.................................................... 122

Coordinated Care Organization C ................................................... 134

Summary of Integration Level Findings ................................................. 144

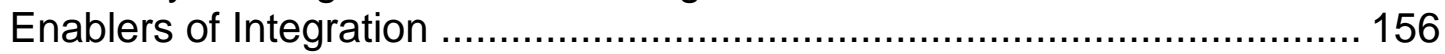

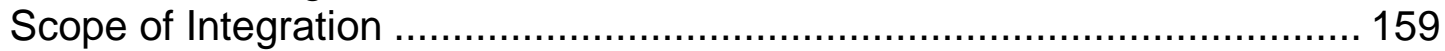

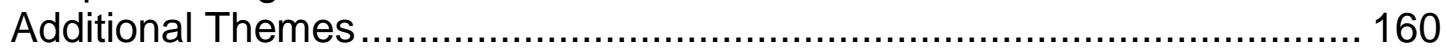

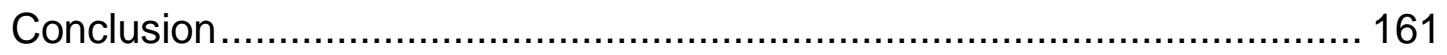

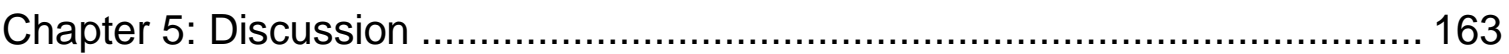

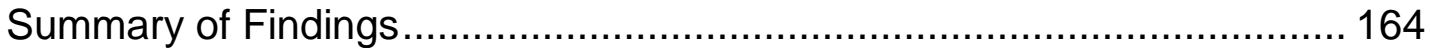

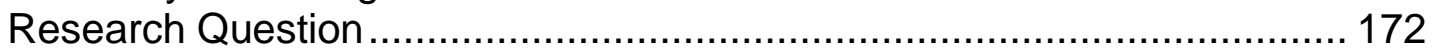

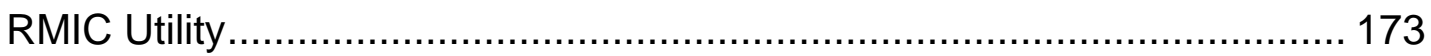

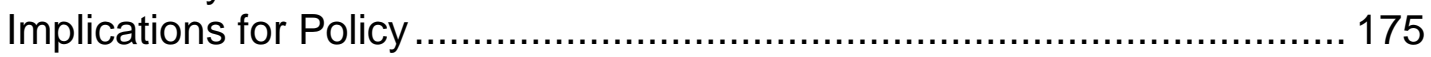

Limitations of the Research .......................................................... 180

Recommendations for Future Research ............................................... 184

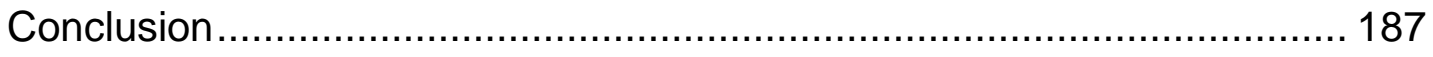

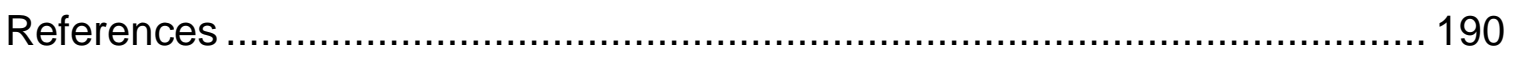

Appendix A: CCO Interview Protocol \& Consent Narrative .............................. 206

Appendix B: Initial Email for Research Participant Recruitment ..................... 209

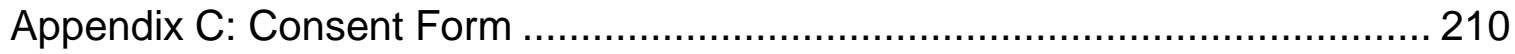




\section{List of Tables}

Table 2.1: PCPCH Standards and Definitions ................................................. 43

Table 2.2: SAMHSA-HRSA Framework of Collaboration \& Integration .............. 51

Table 2.3: Complex Adaptive Systems, the Study of Organizations, \&

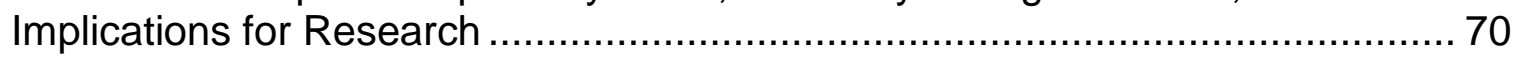

Table 3.1: Definitions of Core Concepts .................................................... 77

Table 3.2: Operational definitions of RMIC domains ..................................... 78

Table 3.3: Interviews and Secondary Interviews, by CCO …........................... 86

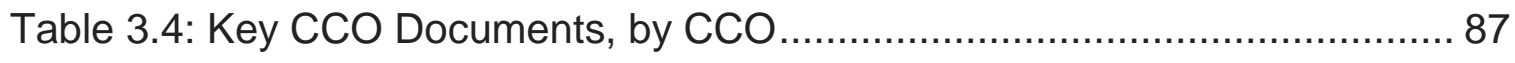

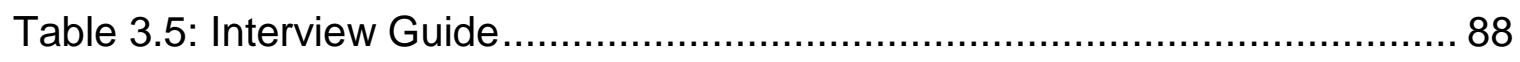

Table 3.6: Coding Scheme and Emerging Themes ....................................... 92

Table 4.1: CCO and Community Characteristics .......................................... 110

Table 4.2: RMIC System Integration, by CCO …....................................... 146

Table 4.3: RMIC Organization Integration, by CCO ..................................... 149

Table 4.4: RMIC Professional Integration, by CCO ….................................. 153

Table 4.5: RMIC Clinical Integration, by CCO …........................................... 155

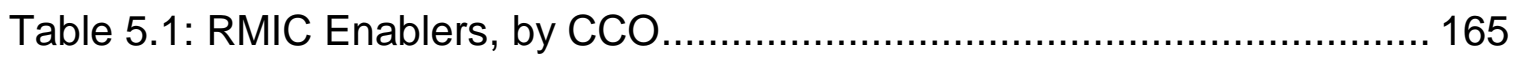




\section{List of Figures}

Figure 1.1: The Rainbow Model of Integrated Care ........................................ 13

Figure 2.1: 4 Quadrant Clinical Integration Model ............................................ 47

Figure 2.2: Collaboration Continuum ..................................................... 50

Figure 5.1: RMIC and the Role of Structural Models .................................. 168 


\section{Chapter 1: Introduction}

Traditionally primary care and mental health systems have operated in silos, resulting in poor communication and lack of coordination (U.S. Department of Health and Human Services [DHHS], 1999; Druss \& von Esenwein, 2006; Horvitz-Lennon, Kilborne \& Pincus, 2006; Institute of Medicine [IOM], 2006). The division of responsibilities for health between these disparate systems often gives rise to duplications, gaps, inconsistencies, and discontinuities in the provision of health care services leading to poor health outcomes and experiences of care as well as high per capita expenditures (DHHS, 1999; Druss \& von Esenwein, 2006; Horvitz-Lennon et al., 2006; IOM, 2006). As a result of multiple barriers to collaboration, patients are frequently forced to navigate a fragmented health care system to receive the care they need.

From a public health perspective, this fragmentation is concerning for multiple reasons. Research indicates that as many as two thirds of patients with mental health concerns are seen in the primary care sector rather than in the specialty mental health care sector (Croghan \& Brown, 2010; Kathol, Butler, McAlpine \& Kane, 2010; Miller, Mendenhall \& Malik, 2009; Blount et al., 2007). Some of these patients have subthreshold mental health conditions that not only make planning and administering treatment for mental illness difficult but also often complicate the presentation and diagnosis of physical health conditions (Kathol et al., 2010). Of these patients, nearly $70 \%$ do not receive mental health treatment and of those who do receive treatment, less than $13 \%$ receive care 
that would improve their outcomes (Kathol et al., 2010). Patients who do not receive treatment or receive ineffective treatment are not only unlikely to see their mental or physical health conditions improve but are also more likely to see their risk for impairment increase.

In addition, individuals with mental illness have higher rates of early mortality and comorbidity (Druss \& Bornemann, 2010). They receive fewer routine preventive services and are more likely to rely on emergency departments for primary care (Baillargeon, Thomas, \& Williams, 2008; Druss \& Bornemann, 2010; Owens, Mutter, \& Stocks, 2010). In 2007, one out of every eight visits to the emergency department in the U.S. involved a diagnosis of mental illness and/or substance use disorder (Owens et al., 2010). By 2013, the rate of emergency department visits involving a diagnosis of mental illness and/or substance use disorder increased significantly; the greatest increase was for mental illness, roughly a $56 \%$ increase for depression, anxiety or stress reactions and a $52 \%$ increase for psychoses or bipolar disorders per 100,000 population aged 15 years and older (Weiss, Barrett, Heslin, \& Stocks, 2016). These emergency visits are thought to be potentially avoidable if these conditions are managed appropriately (Weiss, Barrett, Heslin, \& Stocks, 2016). The combination of the over-utilization of emergency departments and the underutilization of primary care results in expensive and poor quality health care (Pitts et al, 2010). 
In 2015, 43.4 million adults, age 18 and older, were diagnosed with a mental illness that met the criteria outlined by the $4^{\text {th }}$ edition of the Diagnostic and Statistical Manual of Mental Disorders (DSM-IV) (Center for Behavioral Health Statistics and Quality, 2016). Roughly 10 million of those adults had a serious mental illness (SMI), defined as a mental illness that results in impairment that substantially interferes with one or more major life activities. SMI typically includes bipolar disorder, major depression, schizophrenia, and other mental disorders that impose limitations on a person's ability to function on a daily basis (Center for Behavioral Health Statistics and Quality, 2016). For this smaller subset of patients, specialty mental health care clinics may be the only point of contact with the health care system (Druss, 2007). Many individuals with SMI have both cognitive and functional limitations that make navigating a fragmented system of care challenging and may hinder their motivation and/or abilities to seek out needed health care services (IOM, 2006; Druss, 2007; Mechanic, 2007).

Furthermore, comorbidity or the presence of two or more chronic conditions is the rule rather than the exception. The high prevalence of comorbidity and the complex causal connections linking medical and mental health conditions are a driving force behind poor health outcomes and expensive health care (Druss \& Walker, 2011). Comorbidity presents a challenge to the health care system because comorbid conditions are exponentially more complicated and burdensome than the individual conditions themselves (Druss \& 
Walker, 2011). Studies show that mental illness can make it challenging for patients to adhere to treatment recommendations and is associated with increased risk for physical health conditions such as obesity (Hert et al., 2011), chronic pulmonary illness (Jones et al., 2004), cardiovascular disease (Osborn et al., 2007), HIV, Hepatitis B, and Hepatitis C (Rosenberg et al., 2001; Essock et al., 2014). Finally given that only $5 \%$ of the population accounts for almost half of all health care expenditures in the U.S. (Cohen, 2014), gains in cost-savings and quality improvement may have the greatest impact in a smaller subset of the population with the most complex care needs.

As early as 1999, U.S. Surgeon General Dr. David Satcher endorsed the integration of primary care and mental health services as a means to improve quality of care and population health (DHHS). The Institute of Medicine (IOM) followed their infamous report, Crossing the Quality Chasm, with a similar report, Improving the Quality of Health Care for Mental and Substance-Use Conditions: Quality Chasm Series, focused on behavioral health care services (i.e., mental health and substance use disorder services) in the U.S. (2006). Determining that the quality and safety of behavioral health care services are suboptimal, the IOM advocated for better coordination of care for mental health, substance use, and medical conditions (IOM, 2006). Shortly after, the World Health Organization and the World Organization for Family Doctors partnered to publish a paper outlining a comprehensive approach to health care that not only focuses on strengthening 
primary care but also integrating mental health care services in primary care settings (2008).

In addition to advocating for the integration of primary care and mental health services, several agencies have put together resources for promoting and guiding integration. For instance, the Substance Abuse and Mental Health Services Administration (SAMHSA) of the U.S. Department of Health and Human Services provides information, in the form of reports, webinars, and research, about primary care and mental health integration (http://www.integration.samhsa.gov/). The Agency for Healthcare Research and Quality (AHRQ) offers similar resources on their webpage for stakeholders interested in integration (http://integrationacademy.ahrq.gov/).

While the integration of primary care and mental health services has been endorsed by professional organizations, researchers, and other health care stakeholders as one of the most promising approaches for improving the cost and quality of health care for well over a decade, significant barriers have prevented a system-wide effort. The passage of the Patient Protection and Affordable Care Act (ACA) in 2010 offers new hope for addressing the country's increasingly fragmented, costly, and unsustainable health care system. Mental health care was identified as one of the top 10 essential health benefits (45 CFR 156.100). Insurance policies certified and offered in the health insurance marketplace and states that expanded their Medicaid programs must cover these benefits (National Conference of State Legislators, 2013). In addition, delivery 
and payment innovations introduced by the ACA provide opportunities for the provision of mental health services that have not typically been reimbursable, including comprehensive care management, care coordination, social support, and other evidence-based interventions (Lewis et al., 2014).

As policymakers at both the federal and state levels seek to transform health care, attention has turned to how to encourage and facilitate the coordination and integration of care across a continuum of health care needs. The call for integration is predicated on a growing awareness that physical health and mental health are inextricably intertwined and neither can be addressed effectively and efficiently without consideration for the other (Colton \& Manderscheid, 2006). The ACA provides an opportunity to move away from care as usual and instead pursue the integration of primary care and mental health by including provisions that enable states to test and evaluate different financial and organizational models to address system fragmentation.

\section{Background}

While the ACA offers an opportunity for integrating primary care and mental health, much of the work of integration will be done by the states. Thus while it is important to understand the ACA as it provides a framework for state action, potentially both facilitative as well as restrictive, it is not the focus of this research.

One method of state action has been the use of Medicaid; some states are leveraging Medicaid dollars to fund new models of care that promote the 
coordination and integration of health care services (Takach, 2011). This is significant for individuals with mental illness as research demonstrates that Medicaid is the single largest payer of mental health care services (CMS, n.d.). Through Medicaid waivers and various other mechanisms, states are enacting their own interpretations of integration as well as the processes to achieve it (Takach, 2011).

At the forefront of health system transformation, Oregon is expanding on provisions provided by the ACA and taking steps to facilitate the implementation and development of an integrated and coordinated health care delivery system for its Medicaid population in order to achieve what is known as the Triple Aim of improved population health and individual experience of care, with reduced per capita cost of care (Berwick, Nolan, \& Whittington, 2008). Oregon has a long history of health policy innovation and experimentation to increase access to health care and improve population health (Stenger et al., 2012). The passage of HB 2009 (2009) led to the creation of the Oregon Health Authority (OHA) and charged the new organization with the responsibility of implementing the Patient Centered Primary Care Home (PCPCH) program to facilitate the adoption of the PCPCH model, Oregon's version of the Patient Centered Medical Home (PCMH) model (Stenger et al., 2012).

The PCMH model is an enhanced model of primary care that involves the provision of comprehensive primary care services for the purposes of improving communication and shared decision-making among providers, patients, and 
families (Gray, Weng \& Holmboe, 2012; Peikes, Genevro, Smith, Parchman, \& Meyers, 2012; Wagner, Coleman, Reid, Phillips \& Sugarman, 2012). It encompasses the philosophy of integration with its focus on patient/physician relationships, the effective use of teams and community resources, continuity of care, accessibility of care, comprehensiveness of care, and care coordination (Wagner et al., 2012). With its emphasis on whole person care, the integration of primary care and mental health is arguably an essential component in order to provide care that is comprehensive, coordinated, and patient-centered.

In 2011 the Oregon legislature passed HB 3650 establishing the Oregon Integrated and Coordinated Health Care Delivery System and making Coordinated Care Organizations (CCOs) accountable for the provision of integrated and coordinated health care for the Medicaid population (OHA, n.d.a). CCOs are entities that are responsible and accountable for the health outcomes of the population they serve and are governed through a partnership among health care providers, community members, and stakeholders in health systems that hold both financial responsibility and risk (McConnell, 2016). They are given a single global budget and considerable flexibility to deliver defined outcomes. The primary goal and the rallying cry behind Oregon's transformation initiative is the Triple Aim of "better health, better care, lower costs" (OHA, n.d.a; Berwick et al., 2008). While CCOs are not exclusively focused on mental health care, there is considerable emphasis on the importance of integrating primary care and 
mental health and in particular redesigning the system to provide better care at lower costs for individuals with SMI (OHA, n.d.-a).

Currently there are $16 \mathrm{CCO}$ certified by $\mathrm{OHA}$ and each of them is strongly encouraged to integrate a wide spectrum of primary care and mental health services (OHA, n.d.a). Beyond broad directives regarding coordination of health care needs and services and the use of PCPCHs to the greatest extent possible, definitions and models of integration are left to the discretion of individual CCOs. The hope is that this flexibility will: (1) enable CCOs to consider and accommodate local context and needs when devising new ways of organizing, delivering, and paying for care and (2) provide CCOs with room to "think outside the box" and promote the diffusion of innovation within and across ccos.

\section{Research Question}

What key factors in Oregon's health care system impede or facilitate the ability of Coordinated Care Organizations to encourage the integration of primary care and mental health?

\section{Theoretical Framework}

This research drew on multiple disciplines and brings together literature on health policy, health care management, primary care and mental health integration, and complex adaptive systems in order to identify the key factors that impede or facilitate the ability of health care organizations to support and encourage integrated care. While not the main focus of this study, the complex 
adaptive system (CAS) perspective of health care systems provided a general theoretical foundation for this study.

CAS are made up of multiple interacting components and are characterized by uncertainty, complexity, and competing interests (Gioia \& Chittipeddi, 1991; Plsek \& Greenhalgh, 2001). The interactions among different components are typically complex, not easily predicted, and can result in unintended consequences (Plsek \& Greenhalgh, 2001). These features make it difficult to plan and manage change as systems are unpredictable. They are either extraordinarily resistant to change and remain stagnant despite many attempts to affect change or conversely quickly and suddenly change when a tipping point is reached (Gladwell, 2002). Approaches associated with "fixing" problems in simple systems are not effective in CAS when trying to solve complex problems (Plsek \& Greenhalgh, 2001).

Consistent with an open systems view of health care organizations (Pfeffer \& Salancik, 1978; Scott, 2002), the CAS framework challenges the rational model of organizational behavior and posits that real-world organizations are open systems whose behaviors and outcomes are influenced by, and exert influence over, complex and uncertain environments (Plsek \& Greenhalgh, 2001). This perspective acknowledges that solving complex problems requires whole-system, interaction-based, non-linear thinking.

Change in CAS frequently involves a non-linear sequence of events with feedback loops and modifications informed by learning and adjustment (Gioia \& 
Chittipeddi, 1991). Change occurs when organizations transform their current way of thinking and acting in order to facilitate their abilities to take advantage of important opportunities or cope with potential threats (Gioia \& Chittipeddi, 1991). What this suggests is that the change process is influenced by multiple factors and must be actively managed. In order for CCOs to effectively support the integration of primary care and mental health, leadership at both the state level as well as within CCOs will need to actively respond to barriers as well as leverage potential opportunities to their advantage.

The U.S. health care system is at a precipice. Care as usual is unsustainable in terms of the costs, both human and financial. At the same time, policy developments at the national and state levels have laid the foundation and created opportunities for new ways of organizing, delivering, and paying for care. In this new environment, CCOs find themselves presented with extraordinary opportunities while simultaneously confronted by challenges that threaten longterm sustainability. This study seeks to understand the key factors in the health care system that impede or facilitate the ability of CCOs to encourage the integration of primary care and mental health.

\section{Conceptual framework}

While CAS provides a theoretical foundation, the Rainbow Model of Integrated Care (RMIC) provided the framework through which the contextual factors that impact efforts to integrate primary care and mental health were explored. The work to integrate primary care and mental health services is 
marked by both ambiguity and uncertainty. There is no one single definition nor one clearly defined process to achieve it, rather there are multiple definitions and processes. The RMIC developed by Valentijn and colleagues, provides a useful visualization that demonstrates this complexity (Valentijn, Schepman, Opheij, \& Bruijnzeels, 2013). In Figure 1.1., the RMIC framework identifies three different categories, with eight corresponding domains of integration that are situated within the health care system (Valentijn et al., 2013).

First is the scope of integration which can be (1) person-focused and/or (2) population-based (Valentijn et al., 2013). Together they serve as guiding principles for a strong health care system that focuses on providing patientcentered care while considering population health. Second is the type or level of integration which includes (3) clinical integration, (4) professional integration, (5) organizational integration, and (6) system integration. Third are the enablers of integration (7) normative and (8) functional; the absence or presence of these enablers can impact the success of integration efforts. The RMIC draws attention to the fact that integration is a process that can be enacted at various levels of the health care system and it raises questions about how contextual conditions within and across these levels have an impact on systemic efforts to integrate care (Valentijn et al., 2013). 
Figure 1.1: The Rainbow Model of Integrated Care

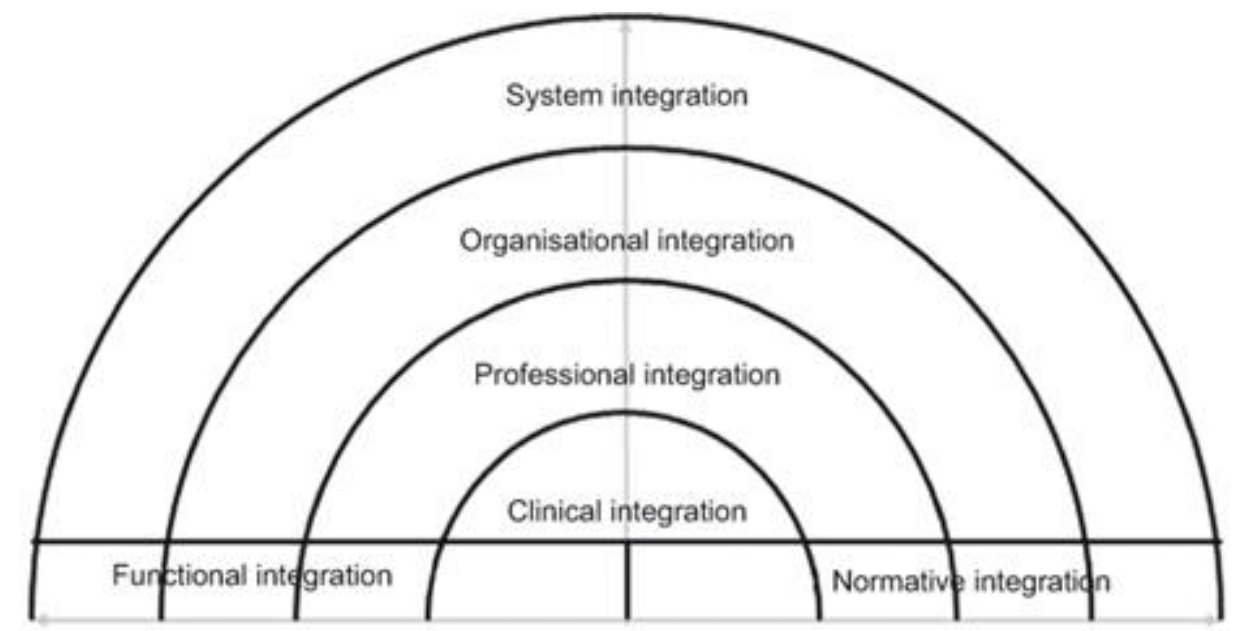

Population based care

Person-focused care

Population based care

Valentijn et al., 2013

Under this framework, integration then is understood to be a coordinated approach to the delivery of health care services that can be supported by processes at multiple levels throughout the system with the ultimate goal of providing comprehensive whole-person care to individuals and populations (Valentijn et al., 2013). Normative and functional enablers of integration speaks to the absence or presence of enablers that not only support integration at any level, but can also serve to connect different levels. Ideally, normative and functional enablers of integration as well as contextual conditions within each level of the health care system are aligned to support integration processes (Valentijn et al., 2013).

\section{Statement of Problem}

As the nation attempts to grapple with health system reform, there is heightened interest in the concept of an integrated health care delivery 
system. This system is conceptualized as a network of organizations that provides or arranges to provide a coordinated continuum of health care services to a defined population and is willing to be held both clinically and fiscally accountable for the health of the population served (Hwang, Chang, LaClair, \& Paz, 2013). The expectation is that organizations will have the capacity to offer more coordinated health care which will result in the Triple Aim (Berwick et al., 2008; Hwang et al., 2013). The concept of health care integration has been around for decades but it has only recently been supported by efforts at both the state and national levels.

The RMIC, unlike previous models, approaches integration not from the clinical standpoint but instead from a more holistic, system level perspective that takes into account the contextual conditions at multiple levels throughout the health care system (Valentijn et al., 2013). It focuses attention on the different types of integration processes as well as acknowledges the role that both functional and normative enablers of integration play in facilitating integration within as well as across levels. It is supported by decades of literature that demonstrates that integration of specialty health care and primary care is complex, affected by multiple contextual conditions, and that those conditions as well as the processes involved in integrating health care need to be carefully managed (Valentijn et al., 2013).

This study attempts to generate understanding, from the perspective of organizational stakeholders, about the capacity as well as the limitations of 
organizations to facilitate integration and explores the factors that impact integration processes across and within different levels of the health care system. In addition, this study will also in part serve to test the RMIC and assess how useful it is as a framework for guiding research on integration efforts undertaken by CCOs. While the RMIC provides a holistic, system level perspective of integration, it is fairly new and has been developed with the goal of being broadly applicable to a wide variety of settings. The domains identified by the RMIC are well backed by the literature; however, further research is needed in order to increase knowledge about the relationship between the domains and assess how interactions between domains and concepts identified by the RMIC may facilitate or impede efforts to integrate primary care and mental health.

\section{Purpose and Significance of the Study}

Oregon is actively pursuing health system transformation in an attempt to transform the way health care is organized, delivered, and financed to improve the quality of health care and population health. While the policy that created CCOs indicates that CCOs are responsible for coordinating and integrating health care, it does not prescribe how that should be accomplished (citation). Considerable flexibility is afforded to CCOs in defining integration of primary care and mental health and establishing how they will facilitate it.

This study aims to ultimately identify which key factors in the health care system shape efforts to integrate primary care and mental health. In doing so, this study also seeks to (1) assess the usefulness of the RMIC as a systemic 
framework to examine primary care and mental health integration, (2) describe how primary care and mental health integration is understood, defined, and coordinated by Coordinated Care Organizations, and (3) explore early lessons for managing primary care and mental health integration in ways aligned with the short-and long-term goals of health reform. This study is significant in that it may provide insights into the capacity and limitations of organizations to facilitate system level change, identify approaches for increasing the capacity of health care organizations to facilitate primary care and mental health integration, as well as help refine the RMIC for practical use as a tool for assessing integration efforts.

\section{Summary}

Mental illness is a huge driver for both increased health care expenditures and poor health outcomes (Druss and Bornemann, 2010). The integration of primary care and mental health has been steadily gaining support as a possible solution to the current fragmented delivery system of care (Pincus, 2003; Kathol, Butler, McAlpine, \& Kane, 2010; Collins, Hewson, Munger, \& Wade, 2010). While the research is convoluted, there is evidence that integrating primary care and mental health services may have positive impacts in terms of access to care, patient outcomes, quality of care, and reduced health expenditures (Pincus, 2003, Kathol et al, 2010; Collins et al, 2010). Recent policies at the federal and state level offer opportunities to restructure the health care system by promoting programs and models of care that encourage and support integration, 
collaboration, and team-based care to more effectively provide patient-centered and comprehensive services.

Despite the growing body of research that indicates that integration may be one of the most promising ways to improve population health and control costs, health care systems have historically struggled to integrate care in a meaningful way. Significant barriers exist at all levels within and across the system. Despite these challenges, federal and state governments are moving forward with efforts to facilitate the integration of primary care and mental health and experimenting with different strategies to organize, deliver, and pay for care in ways that support coordinated and integrated care (Takach, 2011). It is important to assess these efforts and to gain a better understanding about the challenges that health care organizations face in facilitating the integration of primary care and mental health in order to inform policy and advance knowledge. 


\section{Chapter 2: Review of the Literature}

\section{Chapter Organization}

This chapter presents a review of the literature that serves as a backdrop and foundation for the proposed study. It includes a discussion on the following:

1) The case for integrating primary care and mental health;

2) A review of the relevant national policy context that has shaped how mental health care has traditionally been delivered, including the Community Mental Health Centers Act of 1963, Supplementary Security Income, Social Security Disability Insurance, and Medicaid, the Mental Health Parity and Addiction Equity Act of 2008, and the Patient Protection and Affordable Care Act of 2010;

3) A review of the relevant Oregon policy context including the creation of the Oregon Health Plan, the shift to managed carved-out mental health organizations, and bills HB 2009 (2009) which created the Oregon Health Authority and Patient Centered Primary Care Home Program and HB 3650 (2011) which established Coordinated Care Organizations;

4) An overview of the literature clarifying the concept of primary care and mental health integration;

5) A discussion regarding the evidence for the integration of primary care and mental health;

6) A summary on barriers to integration, health system redesign strategies, and the Rainbow Model of Integrated Care; and 
7) A discussion about the complex adaptive systems perspective and how it applies to health care more broadly.

\section{The Case for Primary Care and Mental Health Integration}

The case for integrating primary care and mental health is predicated on growing awareness that fragmentation of health care leads to higher per capita expenditures and poor health outcomes (DHHS, 1999; Druss \& von Esenwein, 2006; Horvitz-Lennon et al., 2006; IOM, 2006). In particular, integration is presented as a promising approach for addressing several related health concerns, including: (1) the majority of individuals with mental illness present in the primary care sector, (2) individuals with serious mental illness (SMI) have worse health outcomes than the general population, and (3) comorbidity is the rule rather than the exception.

In late 1970s, researchers interested in understanding the prevalence of mental health disorders in the U.S. as well as the proportion of patients with mental illness who present at mental health versus primary care settings found that roughly $15 \%$ of the population were affected by mental illness and more than $50 \%$ of those individuals presented in the primary care sector (Regier, Goldberg, \& Taube, 1978). Since then, multiple studies have documented similar findings with anywhere from $26 \%$ to $60 \%$ of patients presenting in the primary care sector for health concerns related to mental illness (Kroenke et al., 1997; Ansseau et al., 2004; Norton et al., 2007). In spite of the fact that primary care providers see 
a significant number of patients with mental health concerns, they are frequently ill-equipped to identify and address mental illness effectively (Mechanic, 2014).

It has also been documented that despite the fact that individuals with SMI utilize emergency and outpatient services at a higher rate than the general population, they receive fewer preventive and primary care services (Salsberry, Chipps, \& Kennedy, 2005; Shen, Sambamoorthi, \& Rust, 2008; Gerrity, 2014; Hunter, Yoon, Blonigen, Asch, \& Zulman, 2015). Research also indicates that individuals with SMI suffer from higher rates of early mortality and comorbidity (Druss \& Bornemann, 2010) and die, on average, 13-30 years earlier than the general population (Colton \& Manderscheid, 2006). In addition, SMI is associated with higher rates of obesity (Megna, Schwartz, Siddiqui, \& Herrera Rojas, 2011; Lopresti \& Drummond, 2013), cardiovascular disease (Gierisch et al., 2013), diabetes (Pendlebury \& Holt, 2010), HIV (Senn \& Carey, 2009), and both chronic and acute pulmonary disease (Jones et al., 2004).

SMI can hinder help-seeking behavior and is frequently associated with higher rates of smoking (Annamali, Singh, \& O'Malley, 2015), poor diet and lack of exercise (Osborn, Nazareth, \& King, 2007; Hert et al., 2011), and comorbid substance use disorders (Brady \& Sinha, 2005), all of which increase risk factors and contribute to poor health outcomes. In addition, the actual treatment for SMI is a contributing factor as well (Robson \& Gray, 2007; Hert et al., 2011). While antipsychotics have enabled individuals with SMI to live relatively productive lives in their own communities, second generation or atypical antipsychotic medication 
has significant negative effects on physical health, increasing risk of obesity, type 2 diabetes, and metabolic syndrome (Toalson, Ahmed, Hardy, \& Kabinoff, 2004; Bhuvaneswar, Baldessarini, Harsh, \& Alpert, 2009).

Finally, comorbidity, the presence of two or more disorders or illnesses, occurring simultaneously or sequentially, in the same person, is the norm (Kessler, Chiu, Demler, \& Walters, 2005; Druss \& Walker, 2011). Findings from the 2001-2003 National Comorbidity Survey Replication indicate that more than $68 \%$ of adults with a mental health disorder have at least one general medical disorder and conversely $29 \%$ of individuals with a medical disorder have a comorbid mental health condition (Druss \& Walker, 2011). However, it is not just the presence of two or more illnesses or disorders that is concerning but rather the interactions between them, which make diagnosis and treatment difficult. Associated with elevated symptom burden, increased functional impairment, and decreased length and quality of life, comorbidity makes it challenging to untangle the overlapping symptoms of the comorbid illnesses and diagnose and treat appropriately (Kessler, Chiu, Demler, \& Walters, 2005; Druss \& Walker, 2011).

The common underlying concern associated with the prevalence of mental illness in primary care, the poorer health outcomes of individuals with SMI, and the complexity of comorbid mental illness and medical conditions, is that fragmented and poorly coordinated health care can impact health negatively as well as make diagnosis, treatment, and recovery exponentially more complicated when comorbid conditions are not identified and treated appropriately. Despite 
advances in science and increasing knowledge about how to recognize, diagnose, and treat mental health conditions, health care systems still struggle with how to adequately address mental illness.

Neither the primary care sector nor the mental health sector has the capacity to meet the full range of health care needs. In addition, the lack of collaboration between these two disparate systems of care puts the onus on patients to navigate a fragmented health care system to receive the services they need (DHHS, 1999; Druss \& von Esenwein, 2006; Horvitz-Lennon et al., 2006; IOM, 2006). This fragmentation of health care results in high costs and poor health outcomes. Integration of primary care and mental health is promoted as one of the most promising approaches for addressing these concerns, improving population health, and controlling costs (DHHS, 1999; Druss \& von Esenwein, 2006; Horvitz-Lennon et al., 2006; IOM, 2006).

Typically, primary care and mental health integration has been a clinical level or an organizational level initiative (Valentijn et al., 2013). Focused on providing care that is both seamless and comprehensive, clinics and organizations have developed a variety of coordinated care models to connect primary care and mental health. Emerging evidence from a variety of these care models has piqued the interest of policymakers and other health care stakeholders in the potential of these models for transforming health care (Druss \& Mauer, 2010). However, research also indicates that characteristics of the health care system can affect the implementation of primary care and mental 
health integration at the clinical and organizational levels (Druss \& Mauer, 2010; Croft \& Parish, 2013).

Historically, health reform efforts have grappled with how to increase access to health care, provide better quality, improve population health, and reduce the overall per capita expenditures (Bim, Brown, Fee, \& Lear, 2003; Fuchs \& Emanuel, 2005). The recent Patient Protection and Affordable Care Act (ACA) of 2010 is no exception, including a number of provisions designed to increase access and quality, while controlling costs (Saloner, Polsky, Kenney, Hempstead, \& Rhodes, 2015). However, the ACA, unlike previous health reform efforts, includes provisions that are intended to increase access to mental health services as well as specifically incentivize the integration of primary care and mental health (Barry \& Huskamp, 2011; Mechanic, 2012; Mechanic, 2014; Saloner \& Le Cook, 2014). As a result several states have taken the opportunity provided by ACA provisions to enact state-level policies that call for new ways of organizing, paying for, and delivering health care services in order to facilitate the integration of primary care and mental health and fundamentally transform health care (Klein \& Hostetter, 2014; Nardone, Snyder, \& Paradise, 2014).

In order to make sense of the recent push for and the challenges of integrating primary care and mental health, it is essential to understand the evolution of the mental health system. The organization, delivery, and financing of mental health services in the U.S. has been shaped by multiple contextual factors embedded within and across different levels of the health care system, 
including normative factors such as leadership, trust, culture, and stigma as well as functional factors such as mechanisms for reimbursement and payment, regulatory frameworks, and information management. This historical background provides the foundation upon which current integration efforts must operate.

\section{Larger National Context}

Federal- and state- level policies as well as the political and environmental context at the federal, state, and local levels have frequently worked to reinforce the current fragmentation of physical and mental health. Concerns about the cost and quality of mental health care have long been important themes, but larger questions regarding responsibility and authority have also played a central role in shaping how mental health services are organized, delivered, and financed. In addition, pervasive stigma as well as a general lack of scientific understanding about the causes of and appropriate treatment for mental illness have often operated at all levels of the health care system in ways that are contrary to achieving overall goals of system reform.

Funding and responsibilities. The history of the organization and delivery of mental health services in the U.S. has revolved around several questions including: (1) who has authority and responsibility, (2) which delivery system models for mental health care are most cost-effective, and (3) what models offer the greatest hope for management and recovery.

Just as medical care once was the sole responsibility of families and local communities, for most of the $19^{\text {th }}$ century, individuals with serious mental illness 
(SMI) were either cared for by families, housed in municipal almshouses, or diverted to prison (Goldman \& Grob, 2006). While states provided funding for the construction of state mental hospitals, local communities were responsible for the actual cost of care and treatment for each patient. This divided responsibility between state and local communities kept the numbers of individuals with SMI in state mental hospitals at relatively low levels. Unless an individual was dangerous to society or his/her family had the means to assume financial responsibility, local officials were incentivized to send individuals with SMI to municipal almshouses and prisons, where costs were far less expensive (Goldman \& Grob, 2006).

Care in municipal almshouses and prisons was at best merely custodial and at worst abusive or neglectful (Grob, 1992). Shocked by the treatment of individuals with SMI, advocates argued that while hospital care was more expensive, it offered a greater probability of recovery and was far more humane in the long run (Grob, 1992). In the mid-1800s, Dorothea Dix, an American activist, conducted an investigation on the state of mental health in the U.S. and brought her findings to the Massachusetts Legislature, where she lobbied for the expansion of the state's mental hospital (Davies \& Janosik, 1991)

It is cheaper to take charge of the insane in a curative institution than to support them elsewhere for life. Well organized hospitals are the only fit residences for the insane of all classes; ill conducted institutions are worse than none at all. Dorothea Dix (Davies \& Janosik, 1991). 
Arguing that almshouses and prisons were not the appropriate sites of care for those with SMI, she traveled to various states across the U.S., making similar appeals, urging states to allocate funds for the construction and maintenance of state mental hospitals with the capacity to provide care that was not only more humane but offered a greater probability of recovery (Davies \& Janosik, 1991). While there was opposition on the grounds that such a venture would increase taxes, advocates for state mental hospitals were able to facilitate an attitudinal shift in what was considered appropriate treatment by increasing awareness of the poor treatment of individuals with mental illness (Torrey, 1997).

By 1880 , there were 75 public mental hospitals in the U.S. (Torrey, 1997). During this time states began to adopt legislation that relieved local communities from the responsibility of providing and financing care for individuals with mental illness. Underlying this policy shift was the belief that while local community care was far less expensive, it was also substandard, inhumane, and costly (Torrey, 1997; Goldman \& Grob, 2006). Centralization was intended to enhance recovery and provide more humane care in state mental hospitals (Goldman \& Grob, 2006). While the purpose of this legislation was clearly to move those with SMI to state hospitals to increase their odds of recovery and potentially realize cost savings, local officials seized the opportunity to shift some of their costs to the state. Municipal almshouses during this time in part served as custodial care for the impoverished elderly and those with serious chronic conditions. Local governments redefined some of these conditions in psychiatric terms in order to 
transfer large numbers of individuals from municipal almshouses to state mental hospitals (Goldman \& Grob, 2006).

Institutional to community care. Moving individuals with SMI to state hospitals was predicated on the notion that the quality of care and treatment was more effective, cost-efficient, and humane; however, the movement of large numbers of the elderly and individuals with chronic disabilities to state mental hospitals changed the quality of care provided (Goldman \& Morrissey, 1985). Critiques of state mental hospitals began before WWII and heightened in the postwar years. Echoing earlier critiques of municipal almhouses and prisons, journalists and advocates alike criticized the poor living conditions, lack of hygiene, overcrowding, ill-treatment, and neglect of patients (Goldman \& Morrissey, 1985).

Notions about community based care began in the early-1900s with the mental hygiene movement, which promoted both the facilities and workforce necessary for community based care by supporting the employment of psychologists and social workers as well as the development of community outpatient clinics and aftercare programs (Vourlekis, 2011). Despite the fact that state mental hospitals were increasingly seen as ineffective at best and abusive at worst, community based care did not take root until the second half of the $20^{\text {th }}$ century. Two key factors greatly increased the political feasibility of community based care: (1) changing perspectives about the root cause of mental illness and (2) the development of psychotropic medications. 
The mental hygiene movement downplayed neurological bases of mental illness and instead stipulated that mental illness was the result of dysfunctional and inappropriate responses to stress or trauma (Cohen, 1983). This idea not only sparked hope that mental illness could be treated but also that it could be prevented (Cohen, 1983). In addition, recent achievements in science, technology, and medicine created an optimistic belief that science could offer a solution for mental illness (Grob, 1992). Those in support of community-based care argued that early identification and the development of promising psychotropic drugs made it possible for individuals with SMI to receive treatment in outpatient facilities embedded within their own communities where they could be supported by family members (Grob, 1994; DHHS, 1999; Sharfstein, 2000; Mechanic, 2007; Novella, 2010).

In 1963 President Kennedy signed the Community Mental Health Centers Act providing federal support for the movement of mental health care services from institutions into community based facilities. This moment marked the victory of an ideology that while grand in vision fell short in practice (Bassuk \& Gerson 1978; Grob, 1994; DHHS, 1999; Sharfstein, 2000; Mechanic, 2007; Gostin, 2008; Novella, 2010). The reduction of admissions to mental hospitals and the release of patients flooded the U.S. with thousands of individuals that communities were ill prepared to support (Bassuk \& Gerson 1978; Grob, 1994; DHHS, 1999; Sharfstein, 2000; Mechanic, 2007; Gostin, 2008; Novella, 2010). 
The shift from institutional to community care was based on several assumptions: (1) patients had a home; (2) patients had family willing and able to assume responsibility for their care; and (3) caring for patients with SMI would not cause undue hardships on family members (Grob, 1992). Advocates for community care neglected to consider the need for transitional care to bridge the gap between hospitalization and community-based care as well as the fact that a large proportion of patients did not have families willing and/or able to care for them.

The role of social welfare programs. The creation of national social welfare programs in the 1950 s to 1970 s, in particular Supplemental Security Income (SSI), Social Security Disability Income (SSDI), and Medicaid, sped up the process of deinstitutionalization by providing a means of subsistence for patients living in community settings. The move to community based care resulted in more than just a shift in the site of care, it also resulted in the loss of housing and food, basic needs that the state mental hospital had traditionally provided. Medicaid played an important role in providing coverage for health care services, but it was SSI and SSDI that made it even marginally possible for individuals with SMI to subsist in the community.

SSDI was enacted in 1956, but it did not become a means of financial support for individuals with mental illness for several more years (Frank \& Glied, 2008). Originally intended to provide cash support for adults aged fifty or older, the program was largely inaccessible by a great number of individuals with SMI 
as these individuals tend to be disproportionately young; four years after enactment, criteria for eligibility were changed (Autor \& Duggan, 2006). The formula for eligibility is complex but, in general, an individual must earn a certain number of work credits within a certain time period before becoming disabled (Autor \& Duggan, 2006). In contrast, in 1975, SSI was established in order to provide stipends to low-income, disabled adults, regardless of work history, as well as disabled children with limited resources and income (Danziger, Frank, \& Meara, 2009). Disability is defined as physical or mental impairment or impairments severe enough to seriously limit the individual's activities (Danziger, Frank, \& Meara, 2009). Together, the two programs offer a means for individuals with SMI that are unable to work to provide for their basic needs.

Medicaid was established in 1965 to provide health insurance for lowincome families and people with disabilities as well as long-term care for poor elderly and disabled Americans (CMS, n.d.). The passage of Medicaid enabled states to shift at least half and sometimes more than half of their costs to the federal government by ensuring patients with mental illness were cared for in community settings (Grob, 1992). Because state and local mental hospitals were seen as primarily custodial institutions as well as a state responsibility, Medicaid was set up to specifically exclude the coverage of services for individuals, age 22-64, in institutions for mental disease (IMD), which were defined as "a hospital, nursing facility, or other institution of more than 16 beds that is primarily engaged 
in providing diagnosis, treatment or care of persons with mental diseases" (42 CFR, section 435.1009).

Under the IMD exclusion, states were incentivized and continue to be incentivized to provide mental health services in community settings in order to maximize federal matching funds (Everett, Sowers, \& McQuistion, 2012). In addition to speeding up deinstitutionalization, Medicaid also fundamentally changed the target population for mental health services by increasing access (Grob, 1992). As a result of improved access to health insurance coverage, increasing numbers of individuals with a wider range of mental health concerns began utilizing mental health services in community based settings (Grob, 1992).

Managed care. Over the years, Medicaid has become a major source of financing for state mental health agencies as well as the largest payer of mental health care services in the U.S. (CMS, n.d.). There is broad variation in the way state programs are administered and states have great flexibility to decide what services will be offered and what populations will be targeted (Randall \& Parente, 2010). During the 80 s and 90 s, in response to the rising cost of health care, managed care developed in the United States as a mechanism for containing health care costs by controlling the delivery system (Lagoe, Apsling, \& Westert, 2005). Originating in the private sector, this approach typically controlled costs and the delivery of care by restricting hospital utilization, such as admissions and length of stay, by limiting access to specialists, and by encouraging healthful behaviors among members (Lagoe, Apsling, \& Westert, 2005). 
Encouraged by the popularity of managed care in the private sector, state governments, burdened with health care expenses for the indigent and elderly, became extremely interested in the approach and began to provide Medicaid services, for both physical and mental health, through managed health care delivery systems as a way to better coordinate care and control costs (Lagoe, Apsling, \& Westert, 2005). Managed care in the context of the Medicaid population has resulted in cost savings, typically achieved through a reduction in the utilization of inpatient care, in fees paid to providers, and in the duration of outpatient treatment (Sparer, 2010). In addition, access to care often improved as well due to the reduction of cost-sharing requirements for members (Sparer, 2010).

States that implemented mandatory managed care for their Medicaid beneficiaries have taken two basic approaches for organizing and financing health care services for mental health conditions (National Alliance on Mental IIIness [NAMI], 2011). In the first approach, the managed care organization (MCO) that provides general medical care also provides mental health care services through its own provider network. This is considered to be integrated care and is far less common than the second approach, where mental health care is "carved out" from managed care arrangements for physical health, and individuals with mental health needs receive mental health services either on a fee-for-service basis or through a separate MCO that specializes in mental health (NAMI, 2011). One of the benefits of carving out mental health care is that it 
ensures that funding is secured and dedicated for mental health services.

However, the unintended and adverse consequence of carving out mental health care has been the reinforcement of fragmentation, with primary care and mental health services delivered in disparate silos with little to no communication between primary care and mental health providers.

Mental health parity. One of the fundamental factors that has shaped mental health care in the U.S. is the pervasive stigma associated with mental illness. Stigma contributes to stereotypes that often depict people with mental illness as dangerous, unpredictable, or incompetent and can lead to discrimination. While much has changed in terms of scientific understanding about mental illness and appropriate treatment, stigma operates at multiple levels within the system to prevent overall health reform goals of improved access, quality, and experience of care.

At the individual level, patients with mental health concerns may avoid seeking treatment to evade real and perceived repercussions of being diagnosed and labelled as mentally ill (Rusch, Angermeyer, \& Corrigan, 2005). In addition, providers may have preconceived notions about the capabilities of their patients with mental illness to make decisions about or adhere to treatment plans; these stereotypes may negatively impact the way providers approach and present treatment options. At the system level, insurers and employers have historically set arbitrary limits on mental health services (Weber, 2013). Coverage for mental health services typically has higher cost-sharing structures, more 
restrictive limits on the number of covered inpatient days and outpatient visits, separate annual and lifetime caps on coverage, and different prior authorization requirements than those found for medical care coverage (Weber, 2013).

The Paul Wellstone and Pete Domenici Mental Health Parity and Addiction Equity Act (MHPAEA) was passed in 2008 and went into effect in 2009 in an attempt to address this disparity in coverage between mental and medical health care services (Dixon, 2009; Weber, 2013). Named after two Senators, both of whom were strong advocates for mental health reform and were very open about their experiences with family members with SMI, the MHPAEA is a federal law that is intended to prevent private health insurance plans that cover more than 50 employees and provide mental health or substance use disorder benefits from imposing arbitrary limitations on behavioral health benefits (Dixon, 2009). This particular issue had been before the Senate multiple times, but significant opposition based on concerns about increased costs and feasibility prevented it from gaining traction. It was only after 37 states (including Oregon) passed their own state-level parity laws that the bill made it through the Senate and became law. While the intention of the law was to protect patients from discrimination in coverage, there were significant limitations; it only applied to group plans and those insurers that chose to cover mental health benefits and enforcement has been challenging (Weber, 2013).

The role of primary care. There is increasing interest in the role of primary care for transforming health care. Despite an overwhelming agreement 
that primary care is a critical foundation for a strong health care system, there is a considerable imbalance between primary and specialty care in the U.S. (Starfield, Shi, \& Macinko, 2005). In the last decade, the Patient-Centered Medical Home $(\mathrm{PCMH})$ has gained significant traction as a potential model to strengthen primary care; it plays an important role in health reform efforts at the federal level as well as within Oregon (Rosenthal, 2008). While it does not focus exclusively on integration of primary care and mental health services, many of the principles underscore the importance of integration (Rosenthal, 2008; Gilfillan et al., 2010). Considering the number of individuals who present in the primary care sector with mental health concerns, increasing the capacity of the primary care sector to address a wide range of health care needs, including mental health, is an important component of health system transformation.

The model typically includes core features such as an emphasis on patient/physician relationship, patient-centeredness, enhanced access, payment reform in alignment with $\mathrm{PCMH}$ values, comprehensive care/whole-person orientation, care coordination, quality and safety, and team-based care (Ferrante, Balasubramanian, Hudson, \& Crabtree, 2010; Markova, Mateo, \& Roth, 2012). Evidence supporting the PCMH includes reductions in hospitalizations, hospital readmissions, and emergency department (ED) visits (Grumbach \& Grundy, 2010); increased patient satisfaction (Jackson et al., 2013); and, better disease management and patient engagement (Peikes et al., 2012). However, there remain questions about whether positive outcomes are the result of unique 
contexts and populations and perhaps not the model itself (Peikes et al., 2012; Rissi et al., 2014).

In addition, the focus of the PCMH model has primarily been on individual clinics, raising concerns about the efficacy of the model when it is expanded to include more broadly a continuum of health care service providers outside the primary care team (Rittenhouse, Schmidt, Wu, \& Wiley, 2013). Similar to any model of integration, expanding the PCMH outside the walls of primary care may face increased challenges for coordination, sustainability and effective teamwork (Rittenhouse, Schmidt, Wu, \& Wiley, 2013). Despite these concerns, both the federal government and Oregon have incentivized the implementation of medical homes as a mechanism for improving coordination and integration.

Affordable Care Act: An opportunity for integration. Two key ACA provisions provide opportunities for addressing system fragmentation: (1) the inclusion of mental health benefits in the Essential Health Benefits package and (2) the application of federal parity protections to mental health benefits (Croft \& Parish, 2013; Kuramoto, 2014; Johnson, Sanders, \& Stange, 2014). Under the ACA, all new small group and individual market plans as well as those states participating in Medicaid expansion are required to cover ten Essential Health Benefit categories. Furthermore, health plans and states expanding Medicaid cannot apply arbitrary limitations on mental health services and instead must ensure that coverage for mental health services is at parity with medical health services (Croft \& Parish, 2013; Kuramoto, 2014; Johnson, Sanders, \& Stange, 
2014). This is notable because the MHPAEA did not originally apply to Medicaid. In 2013, the U.S. Department of Health and Human Services released a statement on the application of MHPAEA to Medicaid MCOs (2015). Parity applies to Medicaid Alternative Benefit Plans regardless of whether the services are provided through managed or non-managed care arrangements. States that carve out mental health benefits are strongly encouraged to ensure that there is parity across the Medicaid delivery system (CMS, 2015).

In addition, the ACA includes several delivery-system reforms that could also help address long-standing system fragmentation (Croft \& Parish, 2013; Kuramoto, 2014; Johnson, Sanders, \& Stange, 2014). The ACA emphasizes integrated care models, such as the PCMH and accountable care organizations (ACOs), which potentially may improve coordination and quality of care. States interested in promoting integration for their Medicaid populations have several options available under the ACA, such as Medicaid "health homes" and colocation grants (Croft \& Parish, 2013; Kuramoto, 2014; Johnson, Sanders, \& Stange, 2014). The Medicaid health homes provide funding for interventions not traditionally covered but with great potential for facilitating integration of primary care and mental health services and improving the quality of care, such as care management, health promotion, post-inpatient transition care, and referral to social support services (Substance Abuse and Mental Health AdministrationHealth Resources \& Services Administration [SAMHSA-HRSA], 2012). These services are reimbursable at a $90 \%$ federal matching rate for the first two years 
following establishment of the health home. Co-location grants available through SAMHSA enable states to integrate primary care and mental health services for adults with comorbid mental illness and medical conditions within communitybased mental health treatment settings (SAMHSA-HRSA, 2012). By taking steps to begin the process of expanding parity and providing mechanisms for encouraging integration, the ACA offers unprecedented opportunity to improve the quality of health care for both the general population as well as for those who receive health insurance coverage through Medicaid.

Summary. The history of mental health policy illustrates the impact of social and political change on the organization, delivery, and financing of mental health services as well as highlights that the current fragmented system, with primary care and mental health services operating in different silos, is embedded within a particular historical context. This historical context offers some broad themes that are important for understanding mental health care more generally but also the recent push for integration specifically.

At a minimum, history illustrates that the system of care for mental illness is complex, impacted by multiple factors, and incredibly difficult to change. Part of this difficulty is inherent in the tension that exists between evidence-based practice and innovation. While the complexity of health care requires innovative and new approaches to address the entrenched fragmentation, at the same time these approaches should be supported by the best available evidence. In addition, historically one of the challenges in mental health care improvement 
and more recently in efforts to integrate primary care and mental health is how to address the needs of the general population as well as subpopulation with serious mental illness and complex comorbid health care needs. Models have been developed and implemented to address the differential needs of both groups but it can be a challenge to focus on one without drawing attention, focus, and funding away from the other. History also highlights that even with the best models of integration and the best of intentions, thoughtful consideration for the mechanisms and systems needed to support as well as monitor change is important. Finally, perhaps the biggest lesson is that even when the intent is clear, policy and transformation efforts can often result in unintended consequences that work contrary to the original goals.

\section{Oregon and Health System Transformation}

Recognized as a national leader in health reform, Oregon has long sought innovative ways to expand access, improve quality, and control costs. In 1991, the state made significant changes to their Medicaid program in order to assure basic health care for all Oregonians (Bodenheimer, 1997). At that time, the eligibility for Medicaid coverage was set at $50-65 \%$ of the federal poverty level and approximately $18 \%$ of Oregonians had no access to health insurance (Mittler, Gold, \& Lyons, 1999). Relying on an innovative public process to determine the definition of "basic" health care, the state developed a prioritized list of acute, primary and specialty health care services. This list was intended to represent the clinical- and cost-effectiveness of the services and was an attempt 
to not only define a "basic" health care package but also address the contentious question of what services should or could be covered without jeopardizing expanded access to basic health care services.

Oregon's section 1115 Medicaid waiver program, the Oregon Health Plan (OHP) was implemented in two year-long phases starting in 1994 (Mittler, Gold, \& Lyons, 1999). In Phase I, OHP eliminated Medicaid's categorical and asset restrictions on eligibility. The program was extended to all legal Oregon residents with incomes below $100 \%$ of the federal poverty level and retained those who qualified under existing Medicaid policy. Phase II included a host of structural changes, but particularly relevant for this study is that mental health services were expanded and moved into managed care through a carved-out arrangement (Mittler, Gold, \& Lyons, 1999).

Central to shaping the organization and delivery of benefits under OHP were political factors at the county level. In order to assuage county concerns that moving mental health care into a managed care contract would destabilize community mental health programs, bidders vying for the MCO contract were required to include local mental health organizations from their potential service areas in the planning process in order to protect the county safety net and ensure the maintenance and stability of viable community mental health programs (Mittler, Gold, \& Lyons, 1999).

Like many state Medicaid programs across the nation, attempts to control costs and improve health outcomes have been limited in Oregon. In 2003, 
economic pressures in Oregon forced the state to restructure Medicaid (James, 2015). Rather than change eligibility to reduce enrollment by the expansion population, the state opted to instead limit benefits costs for this population. Once again applying and receiving a Medicaid waiver, the state created two distinct benefit packages based on the prioritized list. The original benefit package was renamed the OHP Plus and was available to those categorically eligible for Medicaid; the new benefit package was named the OHP Standard and was available for the expansion population (James, 2015).

The OHP Standard reduced benefit costs by imposing premiums and requiring higher copayments (James, 2015). It also seriously scaled back and in some cases eliminated benefits for dental, vision, mental health, and substance use disorder services. Instituting strict premium collection rules, OHP Standard required the automatic disenrollment of individuals for late or missed premium payments and locked them out of the OHP for six months. This attempt at controlling costs had a serious unintended adverse impact; it resulted in the reduction of more than half of enrollees, from 102,000 in 2002 to 51,000 by the end of 2003. Then, in 2004 Oregon voters rejected a tax measure that was intended to help fund the OHP and as a consequence new enrollment in the OHP Standard was closed in 2004. To address some of the problematic policies for the remaining 24,000 enrollees, copayments and the six-month lock out for failure to pay were eliminated and exemption from premiums for those with 
incomes up to 110 percent of the poverty level and a six-month grace period for payment of premiums were instituted (James, 2015).

In 2008, still focused on the goal of expanding coverage, Oregon once again opened its enrollment. However, anticipating demand to be greater than capacity, the state opened a reservation list to help select additional enrollees for the estimated 10,000 available slots (James, 2015). Using a lottery system to randomly select individuals from the list, Oregon continued to use this method to fill available slots until the expansion of Medicaid under the ACA (James, 2015).

In 2009, Oregon passed HB 2009, which was intended to reform Oregon's health care system and achieve the Triple Aim (Berwick et al., 2008; Stenger et al., 2012). HB 2009 (2009) created the Oregon Health Authority (OHA) and charged the new state agency with the responsibility of overseeing all public health care programs within the state, align quality and payment standards, and drive health care delivery system change. As previously mentioned in Chapter 1, HB 2009 also established the PCPCH program in order to facilitate implementation of the PCPCH model, a version of the Patient Centered Medical Home (PCMH) model (Stenger et al., 2012). It also created a 15-member public advisory committee to guide PCPCH policy development.

The committee ultimately selected six core attributes as foundational to the PCPCH model; these are access to care, accountability, comprehensive, continuity, coordination and integration, and patient and family-centered (Office for Oregon Health Policy and Research [OHPR], 2010). See Table 2.1 for the list 
of core attributes and their definitions. With the intention of engaging as many primary care clinics as possible, the committee established a three-tier program. In order to receive recognition, clinics must demonstrate that they meet all of the must-pass standards; beyond that, clinics must accrue a certain amount of threshold points for tier designation (OHPR, 2010).

Early evidence of the medical home model was equivocal (Peikes et al., 2012); however, because the model's emphasis on whole-person, team-based care showed promise for health reform, it was promoted and continues to be promoted as a means for integrating a broad range of mental health care services into primary care (Rissi et al., 2014). In addition, a recent evaluation of the PCPCH program found that the program has facilitated the adoption of teambased and patient-centered care in clinics and saved roughly $\$ 240$ million in its first three years (Gelmon, Wallace, Sandberg, Petchel, \& Bouranis, 2016).

Table 2.1: PCPCH Standards and Definitions

\section{Core Attribute Definition}

Access to care Patients get the care they need, when they need it.

Accountability Recognized clinics are responsible for making sure patients receive the best possible care.

Comprehensive Clinics provide patients all the care, information, and services they need.

Continuity Clinics work with patients and their community to improve patient and population health over time.

Coordination and Clinics help patients navigate the system to meet their integration needs in a safe and timely way. 


\section{Patient and family-centered}

Clinics recognize that patients are the most important members of the health care team and they are ultimately responsible for their overall health and wellness.

The passage of HB 3650 (2011) preceded a long history of health policy innovation. As discussed previously, the bill established the Oregon Integrated and Coordinated Health Care Delivery System and made Coordinated Care Organizations (CCOs) accountable for the provision of integrated and coordinated health care for those covered by the Oregon Health Plan (OHA, n.d.a). The following year in 2012, the Legislative Assembly enacted SB 1580 which expanded on and amended HB 3650 (2011), providing a framework for the implementation of Oregon's new model of care (Oregon Health Policy Board, 2012).

While the statute's stipulation to integrate primary care and mental health is vague, CCOs were instructed to specifically address how they planned to integrate primary care and mental health as well as improve services for individuals with SMI in their transformation plans as well as provides updates on their progress and challenges (OHA, n.d.b; OHA, n.d.d).

Summary. Oregon is moving forward with health system transformation and pursuing a comprehensive reform of its Medicaid health care delivery system through the creation of CCOs, which are both community based and accountable for integrated care. Specific factors unique to Oregon's health care system are likely to have an impact on $\mathrm{CCO}$ efforts to facilitate the integration of primary care 
and mental health. In many ways, Oregon policies as well as its policy-making processes may work to promote the integration of care. Oregon has a history of engaging and involving multiple stakeholders making buy-in more feasible and integration efforts potentially more palatable (Stenge et al., 2012). In addition, the consideration for the importance in accommodating local community needs in the development and implementation of CCOs has provided communities and CCOs with the flexibility to experiment with interventions and strategies for integrating primary care and mental health in ways that consider the needs and assets of individual communities.

However, Oregon health system transformation policies also present challenges to the integration of primary care and mental health. First, while broad stakeholder involvement may facilitate the development of relationships and the trust necessary for health system transformation, Oregon already has experience with the challenge of balancing the interests of multiple stakeholders. Droppers' study on CCOs suggests that the integration of primary care and mental health may depend heavily on the ability of CCOs to develop collaborative relationships built upon trust and reciprocity with the counties and other health care stakeholders in their geographic service areas (2014).

In addition, while the recent focus on health care transformation has led to increased attention to the need for increased integration of primary care and mental health, it has also led to a sometimes confusing array of policies and programs. CCOs attempting to facilitate integration of primary care and mental 
health are caught up in a sea of transformation efforts that may be distracting at best and overwhelming at worst. The potential for change fatigue is high as CCOs, their partners, their clinics, and their providers strive to meet multiple organizational and programmatic standards in an attempt to change the way health care services are organized, delivered, and financed.

Finally, the question of whether metrics are aligned with goals is a paramount concern for health care transformation. Because integration is a complex and abstract concept that encompasses more than just select outcome measures, it is difficult to develop metrics that truly capture the entirety of what integration means and involves. Furthermore, the focus on metrics may make it challenging for CCOs to simultaneously work on short term aims and long term strategic planning.

\section{Clarifying Primary Care and Mental Health Integration}

Despite the burgeoning interest in and increasing calls for primary care and mental health integration, the literature reveals that there is very little conceptual clarity on integration. What integration actually means and what it looks like in different settings and contexts can vary according to a number of factors.

The 4 Quadrant Clinical Integration Model developed by the National Council for Community Behavioral Healthcare (NCCBH) is a useful starting point for understanding what clinical integration is as well as identifying the most appropriate setting for patients with various acuity levels. The model takes into 
account the interaction between risk and complexity of physical and mental health conditions and then provides a description of the integrated care setting best suited to meet the needs of that particular population (Mauer, 2003). See Table 2.1 for the model with brief description of risk/complexity of physical and mental health and ideal setting.

Figure 2.1: 4 Quadrant Clinical Integration Model

\begin{tabular}{|l|l|}
\hline $\begin{array}{l}\text { Quadrant II } \\
\begin{array}{l}\text { Patients w/ high mental } \\
\text { health and low physical } \\
\text { health needs }\end{array}\end{array}$ & $\begin{array}{l}\text { Quadrant IV } \\
\text { Patients w/ high mental } \\
\text { health and high physical } \\
\text { health needs }\end{array}$ \\
$\begin{array}{l}\text { Served in primary care } \\
\text { and specialty mental } \\
\text { health settings }\end{array}$ & $\begin{array}{l}\text { Served in primary care } \\
\text { and specialty mental } \\
\text { health settings }\end{array}$ \\
\hline $\begin{array}{l}\text { Quadrant I } \\
\text { health and low physical } \\
\text { health needs }\end{array}$ & $\begin{array}{l}\text { Puadrant III } \\
\text { health and high physical } \\
\text { health needs }\end{array}$ \\
$\begin{array}{l}\text { Served in primary care } \\
\text { setting }\end{array}$ & $\begin{array}{l}\text { Served in primary care } \\
\text { setting }\end{array}$ \\
\hline
\end{tabular}

Adapted from Mauer, 2003

In two (Quadrant I and III) out of four cases, the 4 Quadrant Clinical Integration Model recommends primary care as the site for service delivery (Mauer, 2003). In both, the primary care provider uses standard mental health screening tools and practice guidelines to provide services to individuals within the primary care practice. The use of mental health screening tools as well as a tracking/registry system enables the primary care provider to refer a subset of the 
population with slightly elevated mental health risk to the mental health clinician. The mental health clinician provides an assessment, brief treatment services, referrals to community and educational resources, and health risk education. In addition the mental health clinician assists the primary care provider with psychiatric consultation, particularly for the purposes of medication management. In Quadrant III, the mental health clinician may also serve as an educator for primary care/medical specialty providers regarding chronic mental health conditions often found in at-risk populations (Mauer, 2003).

In Quadrant II, the recommended site of care delivery is the mental health clinic, where the mental health clinician provides assessment, arranges for or delivers treatment and services, and assures case management for housing and other social support needs (Mauer, 2003). Primary care providers deliver primary care services, but the mental health clinician is responsible for developing a communication process to facilitate coordinated service planning as well as assuring that patients have access to primary care.

Finally, in Quadrant IV, care is best served in a tightly integrated primary/medical specialty care and mental health care system. The primary care provider works closely with medical specialty providers/care managers to manage physical health issues as well as with mental health care providers for the planning and delivery of mental health care services. Psychiatric consultation is fundamental for this particular quadrant due to the high level of risk and complexity. It is important that the medical specialty provider/care manager and 
mental health provider work together as a team to meet the needs of the patient, ensuring that services are not duplicated needlessly or neglected altogether (Mauer, 2003).

The NCCBH notes that the model is intended to be a template, not a prescription. Patients with SMI can be served in any one of the four quadrants as long as the practice develops standard protocols for how acute mental health episodes and high-risk patients will be handled (Mauer, 2003). Implicit in the 4 Quadrant Clinical Integration Model are two dimensions of integration: the direction and degree of integration. Degree refers to how closely primary care and mental health systems work together to provide services, whereas direction refers to whether primary care services are brought into mental health care settings or vice versa.

Degree of Collaboration. Integration can be understood as just one end of a broader spectrum of collaboration (Doherty, 1995; Collins et al., 2010). Along this continuum, collaboration can be: 1) minimal, 2) basic at a distance, 3) basic on-site, 4) close partly integrated, and 5) close fully integrated (Collins et al., 2010). See Figure 2.2 for reference.

At the minimal level, primary care and mental health providers operate under different silos, are located in different facilities, and communicate sporadically (Doherty, 1995). Because providers operate apart from one another and have different administrative and financial systems, it can be difficult to achieve collaborative care. In order to move up to the next level, it is important 
for both primary care and mental health providers to develop clear practice guidelines and standard protocols for screening patients, communicating with each other as well as patients, and making referrals. At the basic at a distance level, providers are still located in separate facilities and operate in completely separate systems, but higher levels of communication and coordination are an improvement over completely disconnected systems. At the basic on-site level, mental and primary health care providers are co-located, share some systems in common, and work together to provide collaborative care. At the other end of the continuum is a fully integrated system, primary care and mental health providers share the same facilities, are a part of the same team, and health is viewed more holistically with mental health care considered an essential component of primary care (Doherty, 1995).

Figure 2.2: Collaboration Continuum

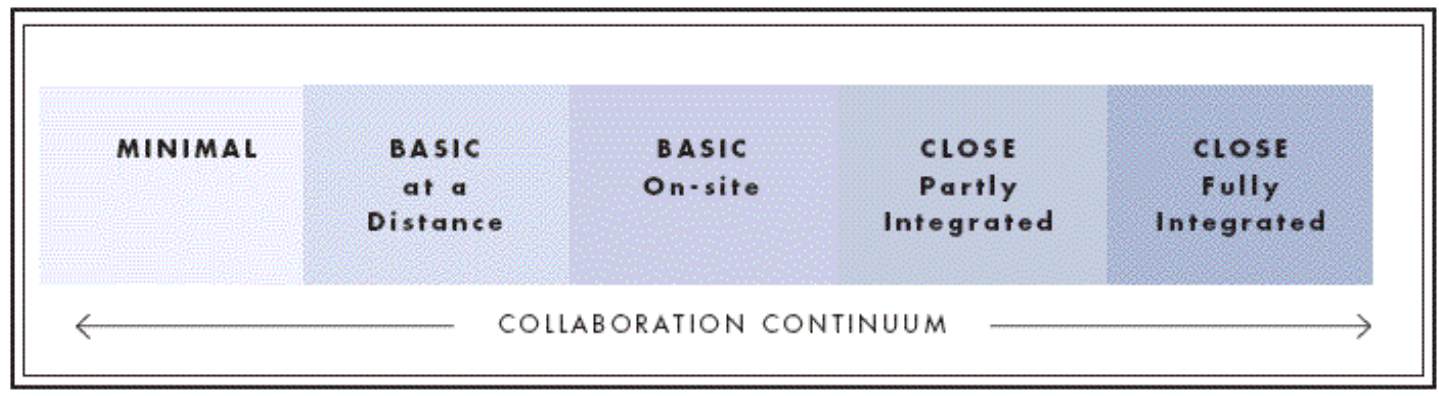

Doherty, 1995

Blount collapsed these five categories into three that define the relationship between primary care and behavioral health services: (1) coordinated, (2) co-located, and (3) integrated (2003). The first, coordinated, refers to primary care and behavioral health services that are coordinated, but 
exist in different settings. The second, co-located, refers to primary care and behavioral health services that are provided within the same practice location. The third, integrated, refers to services that combine primary care and behavioral health services within a single treatment plan (Blount, 2003).

The SAMHSA-HRSA Center for Integrated Health Solutions combined Doherty and Blount's work to create a conceptual framework of collaboration and integration (Gerrity, 2016). Doherty's collaboration continuum is used to define levels of collaboration within Blount's three practice structures to create a sixlevel continuum of collaboration and integration (Gerrity, 2016). See Table 2.2 for reference.

Table 2.2: SAMHSA-HRSA Framework of Collaboration \& Integration

\section{Coordinated care}

Level 1: Minimal collaboration

Level 2: Basic collaboration
Patients referred to another practice site

Providers periodically communicate about shared patients

\section{Co-located care}

Level 3: Basic collaboration on-site communicate but maintain separate cultures and separate treatment plans for patients

Level 4: Close collaboration on site with some system integration and shared records
Providers have some face-to-face communication about shared patients and feel part of a team

\section{Integrated care}

Level 5: Close collaboration approaching an integrated practice 


\begin{tabular}{ll}
\hline $\begin{array}{l}\text { Level 6: Full collaboration in a } \\
\text { merged integrated practice for all } \\
\text { patients }\end{array}$ & $\begin{array}{l}\text { Collaborative treatment planning for } \\
\text { shared patients, but separate planning } \\
\text { for other patients }\end{array}$ \\
& $\begin{array}{l}\text { A team of providers jointly develops a } \\
\text { single treatment plan for patients. } \\
\text { Patients experience their care as a } \\
\text { single system treating the whole person }\end{array}$ \\
\hline
\end{tabular}

Adapted from Gerrity, 2016

Direction of integration. Second, integration can occur in either the primary care or mental health setting. There are valid reasons for either as demonstrated by the 4 Quadrant Clinical Integration Model. With the former, typically the decision to integrate primary care services into mental health care settings is based on a need to provide individuals with SMI access to needed preventive health services (Stozier \& Walsh, 1998). For these individuals, community mental health clinics are often the only point of contact with the health care system. In addition, many of those with SMI have cognitive and functional limitations that may hinder their motivation and/or abilities to seek out and engage in treatment (DHHS, 1999; Mechanic, 2007; Gostin, 2008; Novella, 2010). In order to improve health outcomes amongst this particular population, it has been argued that it is necessary to bring primary care services to them, preferably by bringing primary care providers on-site rather than relying solely on mental health providers to handle and manage primary care services (Stozier \& Walsh, 1998; Druss, 2000).

For the latter, the decision to integrate mental health services into primary care settings is based on a number of factors, including evidence that physical 
conditions are exacerbated by emotional or mental health concerns (Kathol et al., 2010). In addition, a significant portion of the population with mental health concerns relies heavily on the primary care sector (Mauer, 2003; Blount et al., 2007; Miller et al., 2009; Croghan \& Brown, 2010; Kathol et al., 2010). It is also well documented that while scientific understanding about mental illness has grown, mental illness is still highly stigmatized, which may prevent patients from seeking care from mental health clinics (Corrigan, 2004; Mechanic, 2007). Ensuring that primary care clinics offer mental health services may increase the likelihood that people receive needed services for a wide range of mental health concerns.

Scope of integration. One additional dimension of integration that is not captured by the 4 Quadrant Clinical Integration Model refers to the scope of integration. Integration efforts can be focused on a smaller subset of the patient population or more broadly on the general patient population (Blount, 2003). Vertical or specified and targeted programs have their advantages. The research is less ambiguous about the positive impacts of targeted integration programs, particularly for depression (Collins et al., 2010). The targeted approach may also be more feasible than broader approaches due to financial, human resource, and time constraints. However, there are also some downfalls in taking a more specified and targeted approach. The argument for providing integrated care is based on evidence that the mind and body are linked. Focusing more narrowly on specific patient populations or diagnoses runs contrary to this basic 
philosophy. In addition, patients within the wider population, including those with mild to moderate mental health disorders as well as those not diagnosed with specific mental health disorders, may benefit from increased access to mental health services (Kathol et al., 2010).

Summary. One of the challenges in promoting and supporting integration is the lack of conceptual clarity. Integration has become an umbrella term used to refer to a number of interventions intended to bring primary care and mental health closer together. Identifying the factors that impact integration efforts requires understanding how integration is defined and how it is approached. The concepts of degree, direction, and scope of integration provide a way of making sense of integration in order to explore and assess efforts to integrate, identify key factors that shape integration efforts, and monitor, measure, and evaluate outcomes.

\section{Evidence for Primary Care and Mental Health Integration}

Integration can refer to efforts taken by a single clinic or organization to internally assess and address gaps in service needs. It can also refer to initiatives undertaken by multiple clinics or organizations to formally or informally form a partnership through contracts or service agreements in order to provide a continuum of health care services. Integration efforts can also be focused on linking primary care and mental health services for a particular subpopulation or diagnosis or can be broadly applied to the entire population served. This 
conceptual ambiguity makes it difficult to adequately and accurately assess the impact of integration.

Fully integrated systems combine financing, administration, and delivery of care (Shih, Davis, \& Schoenbaum, 2008; Reiss-Brennan, Briot, Savitz, Cannon \& Staheli, 2010). Typically delivering care through teams, these systems have demonstrated increased capacity for detection and reduced burden on providers, patients, and families (Reiss-Brennan et al., 2010). Fully integrated systems vary considerably but most focus on organizational change as the mechanism for transforming care. These efforts are premised on the idea that supportive organizations that manage change and encourage and facilitate people to develop and grow within the context of their relationships with others can effect system change. In a meta-analysis of 21 peer-reviewed articles and 4 non-peer reviewed articles, Hwang, Chang, LaClair, and Paz found that none of the studies actually measured the cost reductions directly but instead used reduction in utilization of services as a proxy (2013). While the evidence for the impact of fully integrated systems on improving the quality of care is rather robust, the evidence for reducing costs is less robust (Hwang, Chang, LaClair, \& Paz, 2013).

More prevalent are attempts by individual clinics and organizations to increase care coordination by integrating mental health into primary care; however, a meta-analysis conducted by AHRQ found mixed results on the efficacy of these efforts (Butler et al., 2008). While some of the studies demonstrate that compared to usual care, integrated care may improve some 
health outcomes for people with depression, these findings are not consistent across all studies. In addition, the length of integration, the use of structured processes of care, and the interaction of the two also appear to have no impact on outcomes. That said most of the studies do demonstrate some improvements in treatment response and remission, but it is difficult to determine what factors led to those improvements (Butler et al., 2008). It is also unclear from the evidence available to determine exactly what elements of integration interventions actually have positive impacts and whether improvements are the result of integrated care or simply any systematic effort to improve processes of care.

Examining the integration of primary care into specialty mental health settings, Druss and von Esenwein's meta-analysis found that integration efforts result in improvements in coordination and medical quality for the intervention group (2006). Results were mixed in regards to medical and mental health/addictive outcomes but this may have been due to the use of different measurements and differences in the relative health of the patients. Studies with control groups consisting of healthier populations did not achieve the statistical significance necessary to suggest intervention impact (Druss \& von Esenwein, 2006). Druss and von Esenwein's review shows promise for the impact of integrated care in specialty mental health settings, but also points to another potential explanation for the less than astounding $A R Q H$ results. Their findings suggest that while integration may improve processes of care, the effects of 
integration efforts are most visible and more quickly realized when applied to the most vulnerable populations with the greatest health care needs (Druss \& von Esenwein, 2006).

There are some limitations in the studies that have attempted to look at the outcomes for integrated care, including fidelity to model and systemic factors, which may partially explain the equivocal findings. In the AHRQ meta-analysis, most notable was that none of the 32 trials implemented all the elements of the model they selected and very few explained how they operationalized the elements they did implement (Butler et al., 2008). Integration is a fluid concept that has multiple definitions. Since there is no single definition of the concept and there is great variability in how it has been implemented in practice, it is not altogether surprising that findings have been mixed. In addition, the system itself is not set up to support primary care and mental health integration and imposes multiple barriers to achieving integration.

\section{Barriers to Primary Care and Mental Health Integration}

The current health care system is not set up to facilitate integration and, in fact, many components actively work against integration goals (Collins et al., 2010; Kathol et al., 2010; Axelsson \& Axelsson, 2006). When primary care and mental health providers operate under different silos, they often face considerable barriers to providing integrated care including the inability to communicate effectively and efficiently with each other due to different organizational, administrative, and financial systems (Collins et al., 2010; Kathol 
et al., 2010; Axelsson \& Axelsson, 2006). Hence one of the arguments for fully integrating primary care and mental health is that full integration may ensure that organizational, administrative, and financial factors are aligned with and encourage collaborative care (Collins et al., 2010).

A review of the literature identifies several key factors that can impact primary care and mental health integration; these elements include (1) organizational structure (2) organizational and professional culture, (3) technology, (4) payment and reimbursement, and (5) regulatory frameworks. These five factors operate in ways that reinforce fragmentation of primary care and mental health and make integration challenging.

Organizational structure. Organizations can be thought of as control systems where the primary concern is how to ensure that individual members complete their tasks for the purpose of achieving organizational goals (Mintzberg, 1993). Organizational structure has significant effects on the level of internal communication, determining the pattern of communication as well as the formal lines of interaction. In addition, organizational structure outlines the policies and procedures that shape decision-making and authority (Mintzberg, 1993; Scott, 2003). The challenge of bringing together diverse organizations is that it typically requires the development of governance structures that promote coordination. To be truly integrated, governance should be diversified, ensuring representation from a variety of stakeholder groups that understand the delivery of health care along its continuum (Savage, Taylor, Rotarius, \& Buesseler, 1997; Trubek, 
2006). The complexity of these systems requires effective mechanisms for accountability and to facilitate decision-making (Friedman and Goes, 2001; Tuohy, 2003; Trubek, 2006).

Organizational and professional cultures. Organizations and professionals also operate according to their own cultures in ways that can impact collaboration and the delivery of team-based care. Providers in primary care and mental health clinics operate under different professional cultures. These professional cultures can be extremely valuable in contexts like health care, in which there are high levels of ambiguity and uncertainty (Abenethy \& Stoelwinder, 1994). Organizations selectively recruit and/or extensively train professionals who will behave "appropriately" because they have internalized the organization's values and goals (Abenethy \& Stoelwinder, 1994). However, this very mechanism also makes collaboration difficult.

Primary care and mental health providers often have different philosophies regarding their roles and responsibilities for providing care (Collins et al., 2010; Pincus, 2003). These different philosophies can impact what providers consider to be a reasonable amount of time to spend with patients, how much information should be included on health records, and the appropriate procedure for communication with other providers (Collins et al., 2010; Pincus, 2003). For example, mental health providers typically spend more time with their patients and may find working within the fast paced environment of primary care settings challenging (Pincus, 2003). In addition, primary care providers who typically write 
concise summaries of patient visits may find the comprehensive write-ups of mental health care providers overwhelming, time consuming, and irrelevant for their needs (Knowles, 2009).

Technology. Technical systems utilized by providers in primary care and mental health settings vary considerably (IOM, 2006). This separation results in challenges in information sharing and communication for both primary care and mental health providers. Health information technology (IT) such as electronic health records (EHRs) can be a key enabler for increased communication. However, mental health care clinics have been slow to adopt information systems for a variety of reasons including concerns for initial productivity losses as staff and providers learn to use the IT system, lack of qualified IT staff, provider resistance, and privacy laws (Drake, Teague \& Gersing, 2005). In addition, even when mental health care clinics do have health IT systems in place, the EHRs used by mental health care providers are seldom compatible with EHRs used by primary care providers. For example, the EHRs used in primary care settings sometimes do not contain data fields for mental health status or mental health care services and vice versa (Collins et al., 2010).

Payment and Reimbursement. Payment and reimbursement structures are often set up in ways that do not reward collaborative care (Horvitz-Lennon, Kilbourne, \& Pincus, 2006; Collins et al., 2010; Kathol et al., 2010; IOM, 2006; Wynia, VanGeest, Cummins \& Wilson, 2003). The traditional fee-for-service reimbursement system often hinders the abilities of primary care and mental 
health providers to work together to provide a more comprehensive set of services. The traditional fee for service system emphasizes volume and medical procedures, neither of which is conducive for mental health interventions and care (Grundy et al., 2011; Collins et al., 2010; Kathol et al., 2010; IOM, 2006; Wynia et al., 2003). Examples of challenges created by reimbursement systems include uncertainty about who to bill for services delivered and low payment for mental health care services delivered in primary care settings (Collins et al., 2010; Kathol et al., 2010). In addition, providers are frequently not compensated for time spent consulting with colleagues, and many services such as patient education and care management have not traditionally been reimbursable (Kathol et al., 2010; IOM, 2006). Recent efforts to transform payment and reimbursement include pay for performance, bundled payments, global budgets, episode-based payments, and shared savings (Rosenthal, 2008; Mechanic \& Altman, 2009). These efforts at payment reform strive to restructure reimbursement to realign payment with value (Rosenthal, 2008; Mechanic \& Altman, 2009).

Regulatory Framework. All health care providers operate within a complex legal framework that can make collaboration challenging (Collins et al., 2010; Axelsson \& Axelsson, 2006). If the relationship is not carefully structured, collaborative activities could potentially be in violation of federal and state antitrust laws (Axelsson \& Axelsson, 2006). Arrangements as simple as referral relationships can raise flags under federal and state fraud and abuse laws. In 
addition, privacy and information laws such as the Health Insurance Portability and Accountability Act (HIPAA) can also limit the ability of clinics to share information and collaborate effectively because it restricts the sharing of mental health information unless strictly authorized (Kahn, Aulakh \& Bosworth, 2009; IOM, 2006). In some cases, state laws can be even more restrictive (IOM, 2006). Even when federal and state laws do not specifically prohibit collaboration activities, the mere perception that they may, can halt collaboration and integration activities. For instance, one response from organizations and providers is to take the most conservative reading of federal and state laws in a preemptive effort to avoid violating regulations.

Summary. Integrating primary care and mental health is a complex problem than cannot be addressed with simple solutions. Fragmentation permeates the entire health care system and is reinforced by both normative and functional factors. While functional factors such as technology, payment and reimbursement, organizational structure, and the regulatory framework are important for aligning all the pieces of the health care system and ensuring that mechanisms are in place to facilitate integration, normative factors such as organizational and professional culture are equally important. Even with all the pieces in place, it is possible to not shift the way that care is provided due to an absence of trust and the proper leadership. Generating buy-in and creating a shared vision and mission for integration that brings the appropriate stakeholders 
together is a precursor to shifting the cultures and practices necessary for primary care and mental health integration.

\section{Health Care System Redesign Strategies}

Much of the literature on primary care and mental health integration focuses on clinical or organizational strategies to better coordinate health care at the site of delivery. Evidence is somewhat equivocal on the benefits of integration but many of these studies note that broader health care system factors frequently work against integration goals, making it difficult to tease out the impacts of integration from a larger system that continually reinforces fragmentation.

Redesigning the health care system requires careful attention to multiple factors that can play a role in either supporting or impeding integration of primary care and mental health. Organizational structure, technology, financing systems, and regulatory frameworks are important for integrating primary care and mental health and making that integration operational by ensuring that the infrastructure is conducive and supportive of integrated care. However, factors such as culture, leadership, and trust, also play a large role by helping to shift attitudes and practices in ways that support integration of primary care and mental health.

With the passage of both the ACA at the federal level and HB 3650 in Oregon, the focus has shifted to transforming the health care system to better support health. Through the alignment of federal and state level policies to promote and support integration, health care system redesign offers a 
perspective that advocates for fundamentally transforming the way that care is organized, delivered, and financed at the system level. These integration efforts are not generally focused solely on integration of primary care and mental health, but rather on broader based health reform. However, these efforts provide an opportunity for primary care and mental health integration, either implicitly by identifying it as a core component for improving health outcomes through coordinated care or more explicitly by outlining specific clinical coordination models to integrate care at the point of delivery.

The lack of conceptual clarity and the resulting equivocal evidence indicates there is considerable need for evaluation when planning and implementing integrated health care systems. Redesigning the health care system requires making changes at multiple levels in order to fundamentally transform the way health is organized, delivered, and financed as well as facilitate integration of primary care and mental health by eliminating the elements that reinforce fragmentation. There are multiple organizational strategies, such as the PCMH model and co-location, which are often used to reorganize care delivery at the site of delivery for the purpose of improving patient outcomes, while redesigning the health system works to remove many of the barriers to integration and aligns incentives as the system level.

In particular, research is needed to identify the key factors and processes that facilitate, as well as those that impede, the implementation of primary care and mental health integration initiatives. Understanding these processes will not 
only help illuminate the most promising strategies for promoting and supporting the integration of primary care and mental health but also the potential relationships among processes and outcomes. According to Suter and his colleagues, the research agenda for integration should include "case studies that document processes, principles, and challenges in planning and implementing integrated healthcare systems within different contexts as well as the roles of the different stakeholder groups" in order to promote conceptual clarity (2009). Such a study would illuminate how key factors in the health care system impact integration activities and provide a stronger foundation for future evaluations that examine the outcomes of these integration efforts. The Rainbow Model of Integrated Care (RMIC) introduced in Chapter 1 provides a conceptual framework that could guide such a study as it situates integration as a multi-level concept that may be impacted by factors within and across various levels of the health care system (Valentijn et al., 2013).

Summary. There are numerous approaches and models of integration, but very little in terms of a conceptual model to guide integration efforts from a systems perspective. The RMIC could potentially offer a systematic way of thinking about, planning, and evaluating integration of primary care and mental health that allows for consideration of the multiple factors that may influence efforts to integrate. 


\section{Complex Adaptive Systems}

Many of the methods used to improve organizational performance are based on Frederick W. Taylor's principles of scientific management, which are based on the assumption that production can be broken into simple step by step procedures. Taylor believed that in order to maximize production, work tasks should be divided into basic steps and training and standardized methods utilized to eliminate differences between workers' performances $(1911 / 2008)$. While this has led to recognition that standardized procedures, training, and incentives can be useful tools for improving performance, it has also promoted the idea that organizations are like machines that receive inputs, transform them, and create outputs through a series of predictable and controllable steps. The machine metaphor has led to misguided beliefs that addressing organizational problems is as simple as identifying the broken "parts" and either fixing or replacing them (Mintzberg, 1993).

However, there is a growing recognition that simple solutions cannot adequately address the complex problems that face health care systems. The study of complexity science offers a useful framework to think about the evolution of complex organizations. It diverges from previous research on organizations by invoking a view of the organization as a living organism, rather than as machine. Complex adaptive systems (CAS) are systems that are made up of multiple agents, who interact with each other and continuously learn and adapt. These 
interactions are typically complex, not easily predicted, and often result in unintended consequences (Plsek \& Greenhalgh, 2001).

Complexity science, or the study of CAS, is not a single theory but rather a body of theories. It has been utilized by biologists, anthropologists, economists, sociologists, and others interested in understanding living systems. In particular, those interested in organizations have focused on using CAS as a way to explain not only how organizations change but also how they can be changed.

Application of complexity science to health care organizations. Four features of CAS are particularly relevant to the study of health care organizations; CAS are thought to be: (1) dynamic, (2) massively entangled, (3) emergent, and (4) robust (Begun, Zimmerman, \& Dooley, 2003). These four features form a theoretical foundation that challenges the rational model of organizational behavior and posits that real-world organizations are open systems whose behaviors and outcomes are influenced by and exert influence over complex and uncertain environments (Plsek \& Greenhalgh, 2001).

Health care organizations are dynamic in that they are made up of multiple stakeholders who interact with each other continuously as well as with contextual factors in the external environment; this continuous interaction results in constant and discontinuous change (Begun, Zimmerman, \& Dooley, 2003). Health care organizations are embedded within other systems (e.g., the local community, the larger state health care system, the national health care system) and the evolution of one system influences the others (Plsek \& Greenhalgh, 2001). The 
boundaries within and between systems and subsystems are fuzzy rather than rigid and stakeholders frequently belong to more than one CAS or shift membership from one to another (Begun, Zimmerman, \& Dooley, 2003).

Relationships and interactions among health care stakeholders within and across health care organizations and the larger system are massively entangled. Through continuous interaction, these agents influence other agents' actions, either by reinforcing stability or facilitating change (Begun, Zimmerman, \& Dooley, 2003). This feature makes it incredibly difficult to plan and manage change as health care systems are extraordinarily resistant to change under some conditions or extremely vulnerable to change under others (Plsek \& Greenhalgh, 2001; Begun, Zimmerman, \& Dooley, 2003). However, while change may be unpredictable that does not mean it is random. While difficult, it is possible to generate generally true and useful propositions about the behavior of health care organizations and the people operating within and across them (Plsek \& Greenhalgh, 2001; Begun, Zimmerman, \& Dooley, 2003).

Health care organizations are inherently self-organizing through simple, internalized rules (Plsek \& Greenhalgh, 2001, Begun, Zimmerman, \& Dooley, 2003). In other words, continuous interaction among stakeholders creates order out of disorder. This self-organizing principle operates at all levels of health care organizations but can be seen clearly in both professions and organizations, which develop their own culture and set of norms that are incredibly resistant to 
change. This resistance to change, or robustness, infuses health care organizations with stability.

Since health care organizations and the stakeholders embedded within and across the system are highly adaptable, even focused efforts to transform the system can result in little change or even unintended consequences (PIsek \& Greenhalgh, 2001; Begun, Zimmerman, \& Dooley, 2003). However, despite the robustness of health care organizations, continuous interaction among stakeholders within and across the health care system can also result in the emergence of innovative behavior and these innovations can spread quickly under the right conditions and in systems where the stakeholders are tightly linked and interact frequently (Plsek \& Greenhalgh, 2001; Rogers, Medina, Rivera, \& Wiley, 2005).

Implications of studying health care organizations as CAS. Diverging from traditional theories about organizational behavior and change, CAS theories assume that the future is relatively unknowable and highly difficult to predict. Historical patterns are considered relevant but not necessarily prescriptive; CAS can have "sensitive dependence on initial conditions," but these initial conditions do not necessarily negate or ensure the possibility of innovation and transformational change from emerging (Begun, Zimmerman, \& Dooley, 2003). Table 2.3 outlines several assumptions and describes their implications for researchers interested in studying CAS. 
Table 2.3: Complex Adaptive Systems, the Study of Organizations, \& Implications for Research

\begin{tabular}{lll}
\hline View of future & Assumption & Implications for Research \\
\hline $\begin{array}{l}\text { Relevance of } \\
\text { history }\end{array}$ & $\begin{array}{l}\text { High, but may not be } \\
\text { deterministic }\end{array}$ & $\begin{array}{l}\text { Requisite to study history, } \\
\text { conduct longitudinal analysis if } \\
\text { possible }\end{array}$ \\
\hline $\begin{array}{l}\text { Domain of } \\
\text { interest }\end{array}$ & $\begin{array}{l}\text { Relationships among } \\
\text { individuals, } \\
\text { subsystems, systems }\end{array}$ & $\begin{array}{l}\text { Study patterns of interaction } \\
\text { among agents, other subsystems, } \\
\text { and the larger environment }\end{array}$ \\
\hline $\begin{array}{l}\text { View of } \\
\text { environment }\end{array}$ & $\begin{array}{l}\text { A domain of interest, } \\
\text { co-evolves with the } \\
\text { organization; mutual } \\
\text { influence }\end{array}$ & $\begin{array}{l}\text { Study the co-evolution of the } \\
\text { organization and the environment }\end{array}$ \\
\hline $\begin{array}{l}\text { Level of } \\
\text { analysis }\end{array}$ & $\begin{array}{l}\text { Nested levels } \\
\text { Strategy }\end{array}$ & $\begin{array}{l}\text { Relatively emergent } \\
\text { View the issue from multiple }\end{array}$ \\
\hline $\begin{array}{l}\text { nested levels of the system } \\
\text { organizational } \\
\text { relationships }\end{array}$ & $\begin{array}{l}\text { Study changes in strategy and } \\
\text { conditions that facilitate change }\end{array}$ \\
\hline $\begin{array}{l}\text { Learning, co-creation } \\
\text { of meaning }\end{array}$ & $\begin{array}{l}\text { Assess degrees of co- } \\
\text { participation, learning, sharing }\end{array}$ \\
\hline
\end{tabular}

Adapted from Begun, Zimmerman, \& Dooley, 2003

A CAS perspective challenges the idea that the organization is the most interesting domain of interest. Rather interactions located inside and outside the organization embedded within multiple, nested levels of continuously interacting systems are the focus of interest. According to Begun, Zimmerman, and Dooley, "the environment is a construct that has little meaning to the complexity 
researcher; rather, relationships among organizational entities and environmental entities are the domains of interest" (2003). In order words, environments do not influence organizations in a linear, top-down fashion; the organization and the environment co-evolve and co-influence each other. Organizations respond to their environments, transforming themselves and the environment in the process. As a consequence, a single level of analysis is not sufficient to understand CAS phenomena. The science of complexity, which emphasizes that systems are embedded within larger systems, fundamentally calls for analyzing relationships across levels of systems.

\section{Conclusion}

The push to integrate primary care and mental health is based on growing awareness that mental and physical health are connected; however, fragmentation is complex and deeply imbedded in the current health care system and has historically been reinforced by multiple factors. For decades there have been greater calls for integrating primary care and mental health and integration has been a key policy priority in numerous mental health reports (DHHS, 1999; IOM, 2006; WHO, 2008). There are isolated examples of successful attempts to integrate and some of these efforts have been ambitious and impressive (Hwang, Chang, LaClair, \& Paz, 2013). However, measured against the backdrop of the larger system where the majority of individuals in the U.S. do not experience high quality, integrated care, these small pockets of improvements have been underwhelming. 
The ACA offers new opportunities for integrating primary care and mental health. It emphasizes the importance of mental health by including it as an essential health benefit as well as by expanding parity in order to increase access to mental health treatment. Oregon is among the leading states moving forward with health systems transformation, developing and implementing a comprehensive reform of its Medicaid health care delivery system through the creation of CCOs. As complex adaptive systems, CCOs and their actors are embedded within other systems and continuously interact with each other as well as the larger health care system. Contextual factors within and across the CCOs will likely impact their ability to facilitate the integration of primary care and mental health and support health systems transformation.

The integration of primary care and mental health more broadly for the general population as well as for those with serious mental illness has been called out as a priority area for CCOs to address; however, there is still great uncertainty regarding what integration means, what it looks like, and how to achieve it. Understanding the factors that influence which approach is implemented and identifying the key factors that impact the ability of health care organizations to facilitate the integration of primary care and mental health would clarify integration and increase the potential for meaningful evaluation. In addition, Oregon's experience with CCOs as they attempt to tackle the problem of system fragmentation can provide early lessons that may help inform other 
states and health care organizations as the nation attempts to bring primary care and mental health together to improve quality and control costs. 


\section{Chapter 3: Methodology}

\section{Chapter Organization}

This chapter provides a detailed description of the qualitative multi-case study research design for the proposed study. It includes the following sections:

1) The main guiding research question and secondary research questions;

2) An overview of the research design as well as the rationale;

3) A description of the conceptual framework and operationalized concepts;

4) discussion about the criteria for inclusion and exclusion;

5) And an overview of the methods for data collection and analysis.

\section{Research Question}

The main research question guiding this study is:

What key factors in Oregon's health care system impede or facilitate the ability of Coordinated Care Organizations to encourage the integration of primary care and mental health?

In exploring the key factors that impact the capacity of CCOs to facilitate the integration of primary care and mental health services, this study sought to seek to (1) describe how primary care and mental health integration is understood, defined, and coordinated by Coordinated Care Organizations and (2) explore the early lessons for managing primary care and mental health integration in ways aligned with the short-and long-term goals of health reform. 
In addition, this study had a secondary purpose. While there are multiple frameworks to guide clinical integration, the RMIC represents the only framework designed to guide and inform systemic approaches to integration. Based on themes that emerge from interviews with CCO stakeholders, observations and field notes, and relevant CCO documents, another aim of this study was to explore the potential of the RMIC and its domains as a useful framework for thinking about integration and providing practical guidance to researchers, policymakers, and other health care stakeholders interested in supporting the integration of primary care and mental health.

\section{Research Design Overview and Rationale}

This study used a qualitative multiple case-study approach to explore Coordinated Care Organization (CCO) stakeholder perspectives on the factors that impede or facilitate the integration of primary care and mental health as well as their thoughts on the relationship between integration and broader health reform efforts. Using the RMIC as a conceptual framework for identifying and organizing a-priori themes, this study collected data from semi-structured and open-ended interviews with CCO stakeholders, observations and memo notes taken during the interviews, and important $\mathrm{CCO}$ documents including the Transformation Plans CCOs were required to write as part of the certification process as well as yearly progress reports and transformation plan updates $(\mathrm{OHA}$, n.d.d). Using this data, the study sought to elucidate the key factors, embedded within and across multiple levels of Oregon's health care system, that 
impact the ability of CCOs to facilitate the integration of primary care and mental health for Oregon's Medicaid population. In addition, this study assessed the usefulness of the RMIC as a tool for evaluating integration.

According to Yin (2013), case-study research is best suited for situations when the researcher wishes to understand a complex, social phenomenon and there is an assumption that important contextual conditions are essential to understanding the case. Given that CCOs and the larger health care system in Oregon are complex adaptive systems, a qualitative case-study approach is the most appropriate research design to explore how interdependencies and interactions among different factors within and across levels of the health care system shape the ability of CCOs to facilitate the integration of primary care and mental health. By utilizing a multiple case-study approach, this researcher was able to look at a variety of interactions and relationships within and across the selected CCOs and the broader Oregon health care system to provide insight into the critical factors that promote or hinder integration.

Initially four CCOs were selected based on a number of criteria. While all CCOs are embedded within the same federal and state regulatory framework, there is great variation among other key factors that likely have an impact on integration efforts. Droppers (2014) study of CCOs identified several key factors that facilitated collaborative governance and decision-making including prior history of conflict or cooperation; open, transparent, and inclusive processes for stakeholders; face-to-face dialogue, trust building, and shared understanding. It 
is probable that these factors shape how CCOs approach facilitating the integration of primary care and mental health as well as how they respond to challenges and opportunities for further strategizing and supporting integration.

\section{Conceptual Framework}

The RMIC provides a framework for exploring how key factors embedded within the larger health care system impact the capacity of organizations to tackle the complex issue of primary care and mental health integration. While the model can be applied to the integration of primary care with any specialty or social care services, it was used in this study to focus exclusively on the integration of primary care and mental health. Thus the core concept of interest is primary care and mental health integration; other concepts of interest come from the RMIC and include scope of integration, type of integration, and enablers of integration. Table 3.1 outlines the important concepts of interest in this study and provides definitions drawn from the literature.

Table 3.1: Definitions of Core Concepts

\section{Concept Definition}

Primary Care \& Primary care and mental health integration is an omnibus Mental Health concept; however, the ultimate goal of integration is to link Care Integration primary care and mental health providers to enhance care coordination. This more comprehensive approach to health care is thought to promote the health of individuals, families and communities (Collins, Hewson, Munger, \& Wade, 2010).

Scope The scope of integration can be specific and targeted or more broadly defined (Valentijn, Schepman, Opheij, \& Bruijnzeels, 2013). Population-based integration is typically focused on a specific demographic population, 
disease, or health issue (Sprague, 2003), while personcentered integration is focused on providing integrated health care for interrelated health concerns that is accessible, comprehensive, continuous over time, and coordinated when patients have to receive care elsewhere (Starfield, 2011).

Type

There are multiple types of integration processes that can occur simultaneously or separate from one another; including clinical, organizational, professional, and systemic integration (Valentijn et al., 2013).

Enablers

Enablers is a broad term that refers to both normative and functional elements; the presence or absence of these elements can facilitate or impede the integration of primary care and mental health (Valentijn et al., 2013).

The eight domains of the RMIC provided the specific framework by which to explore the larger health care delivery system and the factors embedded within and across the levels of the health care system that may have an impact on integration efforts. These have been operationalized based on Valentijn's original definitions and refined through the literature review presented in Chapter 2. Table 3.2 outlines the operational definitions of the RMIC domains.

Table 3.2: Operational definitions of RMIC domains

\section{Concept Definition}

Population-based Population-based care categorizes subpopulations and defines their health care needs according to their burden of morbidity (Sprague, 2003). Integration of primary care and mental health is intended to improve the quality of care and health outcomes for a specific and targeted population (e.g., individuals with SMI, pregnant women, individual with serious depression). The idea behind population-based care is that a specific and defined subpopulation suffers from a greater morbidity and requires targeted intervention in order to improve their health 
outcomes; it often focuses on specific diseases or chronic conditions (Sprague, 2003).

Person-centered Person-centered care considers the personal meaning of illness and takes into consideration personal preferences, needs, and values (Starfield, 2011). Integration is intended to take into consideration individual health needs and ensure that every person has access to a widespectrum of health care services to improve the quality of care and health outcomes for the entire population. The idea behind person-centered care is to offer whole-person care to all patients in the population; recognizing that patients are partners in their own health (Starfield, 2011).

Clinical Clinical integration refers to the extent that care services for patients are coordinated across a continuum of services, conditions, providers, settings, and time in order to improve the quality and experience of care as well as health outcomes (Valentijn et al., 2013).

Professional Professional integration refers to inter-professional partnerships based on shared competences, roles, responsibilities and accountability to deliver a comprehensive continuum of care (Valentijn et al., 2013).

Organizational Organizational integration refers to inter-organizational relationships (e.g., contracting, strategic alliances, knowledge networks, mergers), including common governance mechanisms, to deliver comprehensive services (Valentijn et al., 2013).

System System organization refers to the alignment of both formal and informal rules within a system that facilitate or impede the integration of primary care and mental health (Valentijn et al., 2013).

Normative

Normative enablers of integration refer to internalized rules, linking cultures, and shared values whose presence or absence can facilitate or impede integration (Valentijn et al., 2013).

Functional

Functional enablers of integration refer to the technical and administrative tools which facilitate or impede communication and collaboration (Valentijn et al., 2013). 


\section{Selection of Coordinated Care Organizations}

For the purposes of this study, the CCO provided the frame for analysis and was the unit of study. Each CCO provided an opportunity to explore the relationship between efforts by CCOs to facilitate primary care and mental health integration and various factors across and within different levels of the health care system.

Inclusion and exclusion criteria. There are currently 16 CCOs certified by the Oregon Health Authority $(\mathrm{OHA})$ that vary across multiple characteristics such as geographic service area, number of members served, and governance structure. Each of the CCOs has been tasked by statute to create an integrated and coordinated health care delivery system that includes the specific aim of integrating primary care and mental health services for its members. While all CCOs operate under the same federal and state level regulatory and political structure, there are significant differences specific to $\mathrm{CCO}$ and their local communities.

In effect, Oregon provides an opportunity to examine 16 different organizational approaches to facilitate the integration of primary care and mental health. Exploring all 16 would have provided rich information about the key factors that facilitate or impede integration of primary care and mental health as the system as a whole moves towards change. However, given significant limitations on time and money, this study focused on three CCOs that were selected using the following criteria: (1) meaningful intention to facilitate 
integration, (2) geographic service area, and (3) partnership history. These criteria were applied through several processes including (1) review of Transformation Plans, (2) literature review, and (3) key informant recommendation.

Inclusion and exclusion criteria were applied in several phases. First, to be considered for inclusion, organizations had to be certified as a CCO by the Oregon Health Authority. As of 2015, there are 16 CCOs that vary across multiple characteristics and thus all 16 met this requirement. Second, to be eligible for consideration, CCOs had to be actively seeking to integrate primary care and mental health. In order to assess meaningful intention to integrate primary care and mental health, Transformation Plans were thoroughly reviewed in order to assess and confirm that the CCO had some plan in place for facilitating the integration of primary care and mental health. Third, key informants were asked to provide feedback on the CCOs identified for potential inclusion, specifically offering insights on meaningful intention to facilitate integration as well as likelihood for willingness to participate in research. Meaningful intention to facilitate the integration of primary care and mental health was selected as a criterion for inclusion in order to ensure that data collected were rich and informative. CCOs not engaged in meaningful activity to facilitate the integration of primary care and mental health are likely unable to articulate the factors that impact integration efforts. 
There was also an effort to ensure that the CCOs included in the study are somewhat generalizable to other CCOs and potentially other health care organizations also seeking to facilitate the integration of primary care and mental health services for a defined population. Based on key informant recommendations and the literature review, geographic service area and partnership history were identified as particularly salient characteristics.

CCOs were chosen to reflect both urban and rural communities. This study used the Oregon Office of Rural Health's definition of rural, which is "geographic areas in Oregon 10 or more miles from the centroid of a population center of 40,000 people or more" (Oregon Office of Rural Health, n.d.). Using the zip code list with their rural/urban designation provided by the Oregon Office of Rural Health, the researcher determined whether the CCOs identified for inclusion from the second phase serve primarily zip codes designated as rural or urban. The final selection was made in an attempt to ensure that at least one CCO that served primarily a rural geographic region was included.

As discussed in Chapter 2, complex adaptive systems can be sensitive to initial conditions. While history does not necessarily prescribe whether and how change occurs, its relevance and impact on the evolution of organizations is high, making it necessary to consider the implications of initial conditions. Of particular relevance for the integration of primary care and mental health is partnership history. Some CCOs consist of partnerships among organizations that have extensive histories. In cases where this history has mostly been cooperative, it 
may serve as a facilitating factor and in cases where this history is rife with conflict and struggle, this could serve as an impediment (Droppers, 2014). In particular, $\mathrm{CCO}$ efforts to facilitate the integration of primary care and mental health may be impacted by prior partnership histories with the county mental health departments that have traditionally served as the local mental health authorities in Oregon. In some cases, partnership history does not exist; the absence of a history between or among partnering organizations likely comes with its own set of challenges. Attempts were made to ensure that variation in partnership history was reflected in the case studies selected.

Originally, four CCOs were selected; these four were selected based on the criteria and represented variation in geographic service area and prior partnership history. Ultimately three CCOs were included as case studies. While the researcher reached out to key informants from all four, key informants from the fourth $\mathrm{CCO}$ were not responsive. This is likely due to timing as the fourth CCO was currently undergoing significant restructuring. The researcher considered selecting an alternative $\mathrm{CCO}$ to replace the fourth; however, after conducting fourteen interviews with executives and senior staff from the three CCOs, the researcher decided not to pursue a fourth CCO due to several reasons.

First, securing the interviews with executives and senior staff proved to even more challenging than originally anticipated. Second, the amount of information the researcher obtained from fourteen interview with CCO executives 
and senior staff, eleven secondary interviews, and 24 key documents was more than sufficient to answer the research questions. Third, after spending many months contacting potential participants, scheduling and conducting interviews, transcribing, and analyzing the data, it was clear that the researcher did not have the time and resources necessary to add a fourth. The three CCOs included in this study were selected with an attempt to ensure variation for generalization; however, further research is necessary to assess the applicability of the findings to other types of organizations.

On April 25, 2016, the research study received approval (\#163762) from Portland State University's Human Subjects Research Review Committee (HSRRC), also referred to as the Institutional Review Board (IRB). This study fell into the expedited review category as the research presented less than minimal risk to research participants and confidentiality of all research participants was safeguarded through the use of unique 4-digit identification codes that were used in place of names. In addition, any responses provided during the interview process were not tied to any one individual and were only used to illustrate broader themes pulled from the data. The participants recruited for the study were all adults, were not considered vulnerable subjects, and were able to provide consent.

\section{Data Collection}

This study used data collected from archival documents, semi-structured interviews, field notes, and secondary interview data. The use of multiple 
sources of evidence in this study served two purposes. The first was to alleviate the burden on research participants. Due to the high research interest in CCOs, both state-wide and nationally, many CCO stakeholders have already participated in multiple research studies. In order to avoid aggravating what is likely an increasing sense of study fatigue, the researcher first sought and thoroughly analyzed existing documents, such as CCO Transformation Plans to ensure that the interview process was as streamlined and to the point as possible. Second, using multiple sources of evidence increases the opportunity to develop converging lines of inquiry (Yin, 2013). This triangulation of data helped to strengthen the construct validity of the study.

Primary and secondary interview data. Qualitative interviews were completed with CCO executives $(n=4)$ and senior staff $(n=10)$; these were completed in person or by telephone. In addition, the researcher received permission to include a collection of interviews from a NIDA funded study on CCOs and the integration of care for substance use disorder services (R21/R33 DA035640); eleven interviews from that study met the criteria for inclusion (i.e., interview conducted with an executive or senior staff from one of the three CCOs included in this study).

Secondary analysis enables researchers to utilize an existing pool of data from an earlier study to either answer their own research questions or to supplement their primary data. The NIDA funded study's purpose is to examine the integration of care for substance use disorders; however, many of the 
research participants talk more broadly about behavioral health care services and at times specifically about mental health care. After obtaining permission from the principal investigator and co-investigators and receiving approval for the protocol amendment from PSU's HSSRC, the researcher applied the same criteria to the selection of secondary interviews as she applied to the selection of research participants for primary interviews. Table 3.3 presents the details on the interviews within each CCO.

Table 3.3: Interviews and Secondary Interviews, by CCO

\begin{tabular}{ccccc}
\hline CCO & Executives & Senior Staff & $\begin{array}{c}\text { Secondary } \\
\text { Interview } \\
\text { Data }\end{array}$ & $\begin{array}{c}\text { Total } \\
\text { Interviews }\end{array}$ \\
\hline A & 2 & 3 & 4 & 9 \\
\hline B & 1 & 4 & 4 & 9 \\
\hline C & 1 & 3 & 3 & 7 \\
\hline Total & $\mathbf{4}$ & $\mathbf{1 0}$ & $\mathbf{1 1}$ & $\mathbf{2 5}$ \\
\hline
\end{tabular}

Document selection. The documents selected for inclusion in the analysis were based on key informant recommendation as well as the researcher's knowledge of CCOs. Because the focus was on the CCO perspective and the researcher was interested in being able to have comparable documents across all three CCOs, the following documents were selected for inclusion: the original 2013-2015 and the updated 2015-2017 Transformation Plans, Progress Reports, and Milestone Reports that each CCO was required by $\mathrm{OHA}$ to write and submit as well as a legislative report prepared by each $\mathrm{CCO}$ on the CCO's individual impact on health transformation in Oregon. Documents were analyzed to better understand the approaches each CCO has taken to 
facilitate the integration primary care and mental health. Twenty-five key CCO documents were also collected and included both the original 2013-2015 and the updated 2015-2017 Transformation Plans (TP, $n=6$ ), Progress Reports (PR, $n=9$ ) and Milestone Reports $(M R, n=3)$ required by $\mathrm{OHA}$ as well as legislative reports prepared by each CCO on the CCO's impact on health transformation in Oregon $(L R, n=3)$. Table 3.4 presents the details on the key documents collected.

Table 3.4: Key CCO Documents, by CCO

\begin{tabular}{cccccc}
\hline CCO & TP & PR & MR & LR & Total Documents \\
\hline A & 2 & 3 & 2 & 1 & 8 \\
\hline B & 2 & 3 & 2 & 1 & 8 \\
C & 2 & 3 & 2 & 1 & 8 \\
\hline Total & $\mathbf{6}$ & $\mathbf{9}$ & $\mathbf{6}$ & $\mathbf{3}$ & $\mathbf{2 4}$ \\
\hline
\end{tabular}

Semi-structured, open-ended interviews. Semi-structured, open-ended interviews were conducted with members of $\mathrm{CCO}$ executives and senior staff knowledgeable about and influential in primary care and mental health integration efforts. All interviews were conducted with an interview protocol that included questions developed based on the conceptual framework (refer to Appendix A for the full protocol). Questions primarily probed CCO stakeholder perspectives, knowledge, and insights on system characteristics and priorities as well as enablers and barriers to primary care and mental health integration. The goal of qualitative research is to understand the research topic from the perspective of the research participant; therefore, the interview protocol was semi-structured with mostly open-ended questions with a focus on the particular phenomena of 
interest, in this case, the integration of primary care and mental health. View

Table 3.5 for an overview of the interview questions and how they relate to the RMIC's domains.

Table 3.5: Interview Guide

\begin{tabular}{ll}
\hline Domain & Question \\
\hline Scope & $\begin{array}{l}\text { What is your strategy for the integration of primary care } \\
\text { and mental health services? Who is the target } \\
\text { population for [CCO name]'s strategy for integrated } \\
\text { primary care and mental health services? }\end{array}$ \\
\hline $\begin{array}{ll}\text { Organizational, } \\
\text { Scope, Type }\end{array}$ & $\begin{array}{l}\text { Please talk about the process for developing the } \\
\text { integration strategy. What led to [CCO name] choosing } \\
\text { this particular strategy? Has that evolved? If so, how? }\end{array}$ \\
\hline Organizational & $\begin{array}{l}\text { What organizations are involved in the strategy to } \\
\text { integrate primary care and mental health? Please } \\
\text { describe those relationships. Are they different from } \\
\text { how they were in the past? What mechanisms are }\end{array}$ \\
& $\begin{array}{l}\text { utilized to develop and maintain those partnerships? } \\
\text { (e.g., inter-organizational agreements, payment reform) }\end{array}$ \\
\hline Clinical (Type) & $\begin{array}{l}\text { How does the CCO strategy intend to change the way } \\
\text { patients receive services in order to improve or achieve } \\
\text { integration? }\end{array}$ \\
\hline $\begin{array}{ll}\text { Professional } \\
\text { (Type) }\end{array}$ & $\begin{array}{l}\text { How does the strategy intend to change how MH/PC } \\
\text { work together? }\end{array}$ \\
\hline System (Type) & $\begin{array}{l}\text { What role, if any, does [CCO name] play in ensuring that } \\
\text { the specific elements of your integration strategy are in } \\
\text { place or occur? What is the state doing that helps or } \\
\text { hinders your efforts to facilitate integration? In what } \\
\text { ways have state-level policies impacted integration? }\end{array}$ \\
\hline Enablers & $\begin{array}{l}\text { What have been the biggest barriers to managing the } \\
\text { integration of primary care and mental health? } \\
\text { How does [CCO name] manage these barriers? } \\
\text { What have been the biggest factors that have facilitated } \\
\text { the integration of primary care and mental health? }\end{array}$ \\
\hline
\end{tabular}


In the perfect world, what would you change to make integration more feasible? What else would you like to do?

\begin{tabular}{ll} 
Type, Enablers & $\begin{array}{l}\text { What are some of the biggest accomplishments [CCO } \\
\text { name] has accomplished in terms of integrating primary } \\
\text { care and mental health? Are there any stories that } \\
\text { exemplify [CCO name's] role in facilitating integration } \\
\text { that you are willing to share? }\end{array}$ \\
\hline Enablers & $\begin{array}{l}\text { What would you change about the environment to make } \\
\text { integration more feasible? In the perfect world if the } \\
\text { environmental conditions were already aligned to } \\
\text { support integration, what else would [CCO name] do to } \\
\text { facilitate the integration of primary care and mental } \\
\text { health? }\end{array}$
\end{tabular}

Interview process. Potential research participants were recruited via email, which included the consent form that detailed the study and the purpose of research as well as their rights as research participants. Refer to Appendix B for the recruitment email and Appendix $\mathrm{C}$ for the consent form. Before the interview was actually conducted, the researcher read from the consent form which outlined the nature of the study and the voluntary status of participation. They were also informed that the study was confidential and any information shared would not be connected to their names. The researcher secured permission to audio-record the interviews in order to accurately capture participants' words and perspectives. Participants were asked to sign the consent form if they consented to participation.

Interviews were face-to-face when possible. These face-to-face interviews occurred at the participants' host organizations for their convenience. 
Due to scheduling conflicts and geographic barriers, phone interviews were utilized in some cases. Immediately following each interview, the researcher set aside time to take detailed field notes about the interview, documenting interesting insights or questions raised by the interview. All recordings, transcriptions, and other data were then stored on a password protected computer accessible only by the researcher.

\section{Data Analysis}

After the data were collected, the data were analyzed in multiple cycles.

Qualitative data analysis is an ongoing, iterative process that begins in the early stages of data collection and continues throughout the study. The researcher's field notes served as a starting point to begin the analysis process. One of the aims of the study was to assess the usefulness of the RMIC for understanding health care integration. A potential drawback of using the RMIC as a framework to guide the study was that the researcher could potentially rely too heavily on the model, resulting in confirmation bias. The field notes served in part to help prevent confirmation bias, by providing a structured opportunity for the researcher to note interesting and surprising insights that arose during the interviews. All interviews were then transcribed using Microsoft Word within onetwo weeks of completing the interview; each transcript was then assigned a unique 4-digit code. Transcription documents, along with CCO documents, were uploaded into Atlas-ti for coding and thematic construction. 
Coding Process. The focus during the analysis was to identify as well as compare and contrast emergent themes, patterns, and trends within and across the CCOs. The researcher developed a list of a-priori codes informed by the RMIC. However during the data collection process, additional codes were developed as new topics or ideas of interest emerged.

Using the provisional coding method, a-priori codes were developed based on the RMIC and the extensive literature review; codes were expanded as necessary when new themes and ideas emerged (Miles \& Huberman, 1994). The first task during the coding process was to apply primary codes to the data, representing the three categories of the RMIC: (1) scope of integration, (2) type or level of integration, and (3) enablers of integration. The next step was to apply the secondary codes related to the eight domains of the RMIC: (1) personfocused, (2) population-based, (3) clinical integration, (4) professional integration, (5) organizational integration, (6) system integration, (7) normative enablers, and (8) functional enablers.

After completing this initial set of codes, the researcher applied the elaborative coding method to further analyze the data. The focus of the analysis was to identify as well as compare and contrast emergent themes, patterns, and trends within and across the CCOs. Using an elaborative coding method, the researcher identified and characterized the key features of the RMIC integration types at each level and the normative and functional enablers of integration embedded within and across Oregon's health care system. This process 
enabled the researcher to develop a more comprehensive understanding about the relationship between contextual conditions and integration efforts.

Indicators were initially pulled from Valentijn's RMIC taxonomy. Due to emerging themes, new codes were added during analysis; these themes included: (1) Structural model to guide the integration of primary care and mental health, (2) Meaningful metrics to measure integration, (3) Setting in which people are best served, and (4) Equally developed systems of care for primary care \& mental health. See Table 3.6 for a comparison of the initial coding scheme and the final revised coding scheme. After the data were coded, the researcher analyzed these themes in relation to the central and secondary research questions.

Table 3.6: Coding Scheme and Emerging Themes

\begin{tabular}{|c|c|c|}
\hline \multicolumn{3}{|c|}{ Coding Scheme } \\
\hline \multirow[t]{4}{*}{ Type } & Clinical & $\begin{array}{l}\text { - Case management } \\
\text { - Continuity } \\
\text { - Interaction between professional \& } \\
\text { client } \\
\text { - Individual multidisciplinary care plan }\end{array}$ \\
\hline & Professional & $\begin{array}{l}\text { - Inter-professional education } \\
\text { - Agreements on interdisciplinary } \\
\text { collaboration } \\
\text { - Value creation for the professional }\end{array}$ \\
\hline & Organizational & $\begin{array}{l}\text { - Inter-organizational governance } \\
\text { - Inter-organizational strategy } \\
\text { - Trust }\end{array}$ \\
\hline & System & $\begin{array}{l}\text { - Alignment of regulatory frameworks } \\
\text { - Environmental climate }\end{array}$ \\
\hline Enablers & Normative & $\begin{array}{l}\text { - Shared vision } \\
\text { - Reliable behavior } \\
\text { - Visionary leadership } \\
\text { - Linking cultures }\end{array}$ \\
\hline
\end{tabular}




\begin{tabular}{lll}
\hline Functional & - & Learning organizations \\
& - Information management \\
& - Regular feedback of performance \\
& indicators \\
\hline $\begin{array}{l}\text { Themes that } \\
\text { emerged }\end{array}$ & - Structural model to guide integration of \\
during & primary care and mental health \\
analysis & - Meaningful metrics to measure \\
& integration \\
& - Setting in which people are best served \\
& - Equally developed systems of care for \\
& primary care \& mental health \\
\hline
\end{tabular}

After the data were coded, queries were run to explore the relationships among the themes and to assess how normative and functional enablers of integration impact the ability of CCOs to facilitate the integration of primary care and mental health. The researcher analyzed these themes in relation to the central and secondary research questions.

\section{Summary}

Contextual conditions and factors within and across different levels of Oregon's health care system may provide opportunities or impose barriers for CCOs attempting to facilitate the integration of primary care and mental health. The proposed qualitative multiple case-study research used the RMIC to explore relationships between contextual conditions within nested levels of the larger health care system and how those relationships shape integration efforts. Data were analyzed to identify themes and help inform the RMIC to develop a more comprehensive understanding about the key factors that shape efforts to integrate primary care and mental health as well as provide guidance to 
policymakers and other health care stakeholders interested in facilitating primary care and mental health integration. 


\section{Chapter 4: Presentation and Analysis of Data}

The purpose of this study was to explore the integration of primary care and mental health in the context of Oregon's Coordinated Care Organizations (CCO). The main research question guiding this study was: "What key factors in Oregon's health care system impede or facilitate the ability of Coordinated Care Organizations to encourage the integration of primary care and mental health?" In addition, this study had a secondary purpose to assess the usefulness of the Rainbow Model of Integrated Care (RMIC) for exploring integration efforts.

While the RMIC provides a more holistic, systemic view of integration, it is fairly new and was developed to specifically assess the integration of specialty services in primary care settings with the goal of being broadly applicable to a wide variety of countries. Using the RMIC framework, CCO stakeholder perspectives were examined to identify the challenges and opportunities for integrated care, embedded within and across multiple levels of Oregon's health care system (i.e., system, organizational, professional, and clinical).

\section{Organization of Findings}

The findings section provides descriptions of each case based on analysis of primary and secondary interview data as well as key CCO documents. Three cases were explored through the lens of the RMIC framework, paying particular attention to the level of integration processes (i.e., system, organizational, professional, and clinical) and the enablers of integration (i.e., normative and functional). The findings are organized, accordingly: 
1) Findings related to scope of integration,

2) Findings for system integration, all three CCOs are embedded within the same system and there were few differences in their accounts of processes and factors at that level;

3) CCO specific findings, organized according to the remaining levels (i.e., organization, professional, and clinical);

4) A summary of the findings for the integration levels (i.e., system, organization, professional, and clinical) with a focus on similarities and differences across the three CCOs,

5) A discussion about the enablers of integration (i.e., functional and normative) comparing and contrasting the CCOs; and

6) An account of the findings that emerged from the data but were not predicted or accounted for by the RMIC framework.

\section{System Integration}

At the system level, the RMIC framework defines integration processes as, "a coherent set of (informal and formal) political arrangements to facilitate professionals and organizations to deliver a comprehensive continuum of care for the benefit of people and populations" (Valentijn et al., 2015). Included in this set of arrangements are regulatory frameworks and a social, political, and economic climate that supports team-based care and collaboration (Valentijn et al., 2015). System level integration processes are critical for setting the stage for integration processes at the organizational, professional, and clinical levels. 
The first subsection provides an overview of the environmental climate and discusses the following themes: the role and limitations of incentive metrics, the PCPCH model provided a framework, integration in a multi-payer system is challenging. The second subsection focuses on unique findings related to integrating primary care into mental health settings and discusses the following themes: the instability of the mental health system, the lack of relevant incentive metrics, and the potential of a federal program to advance comprehensive behavioral health care.

Environmental climate. The foundation for broader health transformation in Oregon began well before the development and passage of CCO legislation. In 2009, the creation of the Oregon Health Authority (OHA, 2010) itself was an effort to facilitate integration at the system level to break down long existing and persisting silos that divided health and human services (OHA, 2010). The hope and intention was that by bringing all health and human services-related departments under the umbrella of the OHA, collaboration among departments would be both culturally and structurally supported (Rissi et al., 2014).

The genesis of CCOs coincided with federal health transformation efforts as well as other state-wide efforts to transform health, and provided a platform for change. While integration of primary care and mental health is only a piece of the much larger effort to achieve the Triple Aim in Oregon, many of the early conversations and overall narrative focused on integrated care as a critical 
element of, and foundational to, health transformation (OHA, n.d.a). Larger health care system concerns such as budgetary constraints and poor population health outcomes helped to create an overall sense of urgency but interview data indicate that Oregon health policy leaders played an active role in framing health transformation narratives and made it possible to generate the necessary buy-in and commitment.

Enthusiasm for, and commitment to, change was largely facilitated by visionary leadership, led by a governor experienced with and knowledgeable about health policy.

I thought Governor Kitzhaber was amazing. He was such a strong visionary and had such an influence on how the system worked. And his right hand guy was Goldberg and together they were an amazing duo; they were very solid health care leaders. Just having that constant feedback from our leaders about how and what CCOs were doing; I think it was like having bumpers and you knew you could bump into the idea guys and then you could correct course if needed. (ID 0106)

This strong leadership with opportunity for feedback and guidance appears to have played an important role in overcoming initial resistance to change. That said, transitions at OHA, starting roughly in early 2015 and culminating with a restructuring of the agency by 2016 , led some CCO key informants to feel as if "there is no one steering the ship" (ID 0304). Several key informants indicated they would like more guidance from OHA, particularly around mental health and substance use disorder issues as well as around the development of alternative payment methodologies. 
And that goes back to OHA trying to figure out what the agenda is, who's in charge, you know. Addictions and mental health has disappeared. And [Name] has even resigned. And she was as close as we had to a policy person to help us. So I don't know what their plans are. But we don't really have allies [at OHA] to help us with addictions and mental health work. (ID 0101)

It's incredibly tough nut to crack in terms of the financing. The clinical piece is much more straightforward. This is a good step towards that but you know ultimately if we come up with an APM and it doesn't have the actuarial data that they feel they need to share with CMS to show that Medicaid dollars are being spent on specific services and therefore say nope that won't work, that's going to be a problem. While we have these global budgets, if CMS comes down on OHA to say well tell us what you're spending that money on and we don't have the right number of widgets, that's going to be an issue, but the global budget is supposed to allow you to not spend on widgets but to focus on outcomes so that's the conundrum. So it'd be good to get some guidance from OHA on that piece. (ID 0201)

With the resignation of the OHA director in 2014, the resignation of the Governor in 2015, and significant staff turnover and agency restructuring at $\mathrm{OHA}$ throughout 2015 and early 2016, some key informants felt that there was an absence of health policy leadership at the state level. However, there was also a strong narrative that framed health transformation as highly dependent on community context, priorities, and resources.

$[T]$ here are so many ways that integration can look and knowing what's right for a particular population or for a particular $\mathrm{PCPCH}$ clinic and for a particular community... It's really challenging but to some degree people have to figure it out on their own. What are those needs and what's it going to look like for us? (ID 0102)

Thus, according to some CCO key informants, while guidance and feedback from the state were welcome, the actual task of figuring out what health transformation 
looked like on a local level and how to achieve it was simply something that CCOs and their stakeholders had to work out on their own.

Incentive metrics. In order to hold CCOs accountable for access and quality, a number of process and outcomes measures were selected by a committee of nine appointed by the OHA director and tied to incentive payments; of those a few were related to mental health (e.g., depression screening and follow-up and 10 days follow-up after hospitalization for mental illness) (OHA, n.d.c). While key informants had various opinions regarding the incentive metrics, overall most felt that the metrics were helpful in generating the energy and focus necessary for change and in turning attention to the importance of mental health care.

And the metrics have actually changed how we think about our work. Right...I do think that it's changing the way our providers think; [the way] our planned partners think. It has had the impact that they wanted it to have, in that regard. (ID 0105)

I think the state has put some metrics in place that start to push towards integration, so [the metric for] depression screening at least pushes the idea that primary care should be doing that work. It has been helpful in at least [the metrics] take the onus off the $\mathrm{CCO}$ to have to say that integration is important or to have to make that case; it's just sort of baked in which I think is really pretty important. (ID 0102-S)

Key informants noted that the metrics played an important role in generating awareness and recognition of the prevalence and importance of addressing mental health. Given the historical context of primary care and mental health operating in silos and persisting stigmatization of mental illness, this may have been particularly important in primary care settings. Noting that the quantity and 
quality of conversations about mental health and integration increased as people shifted from asking why they had to integrate primary care and mental health services to how they were going to integrate primary care and mental health services, informants indicated that many of these conversations were driven by pressure to achieve metric benchmarks.

That said, key informants were not without skepticism and many had mixed feelings about the metrics. In particular, some questioned whether the metrics selected were meaningful measures of integrated care, while others expressed concern for how they were going to collect data to measure certain metrics.

They're okay; I mean it's good to have metrics related to mental health or behavioral health, there are several metrics related to addiction as well. They do push clinics to focus on behavioral health to get those dollars. But I question if they measure integration. I'm not sure what the right measures for that are but...I think maybe we should have a discussion about what it means to have integration, what does that look like, and how do we measure it. (ID 0301)

There was a ton of resistance...not because people don't think it's important, but because people said, well, we don't know how to measure that. We don't know how to collect data for that. And you know, as one of the only mental health people, I was saying, that's exactly why we need to measure it. (ID 0101)

Finally, others were concerned that not all clinics and providers honored the intent of the metrics in practice. In other words, there were concerns that the metrics may have also produced some unintended consequences such as fixation on meeting the metric itself rather than improving the performance towards attaining the goal of the metric. 
Well...l'm not positive that everyone is really working towards the intent of some of the metrics. Follow-up after hospitalization for mental illness, take that one. The purpose is to ensure that the patient, the individual is connected with care in the community to ensure that they don't end up back in the hospital shortly after being discharged. But l've heard of providers doing the follow-up as they're discharging the patient. What?! So if you're doing that, is this about what is best for the patient? (ID 0202)

Key informants raised concerns that activities to meet the metrics were implemented that do not actually improve the quality of care and that some providers and clinics have responded to the metrics as if they are boxes to be checked,. This concern was more broadly related to the notion that metrics sometimes promoted "teaching to the test" (ID 0301), which can distract from long-term goals and objectives, such as integrating care for the purpose of improving access, quality, and outcomes.

The PCPCH program. When asked to discuss the integration of care, most key informants focused on the integration of mental health services into primary care settings. According to CCO key informants, much of the focus as well as the accomplishments have been in primary care settings. In addition, CCO key informants reported that the Patient Centered Primary Care Home $(\mathrm{PCPCH})$ model was particularly useful in their efforts to facilitate the integration of mental health services in primary care settings.

One of the strengths of the PCPCH is it just gives you a nice model to kind of wrap your head around changing care in the clinic. And it gives you a strong foundation for integrating behavioral health services because of its focus on team based care and comprehensive services. (ID 0301) 
Analysis of interview data and key $\mathrm{CCO}$ documents indicated that the $\mathrm{PCPCH}$ model played a role in the integration of care for two primary reasons. First, the PCPCH model reflects the philosophy of integrated care with its focus on whole person care. Specifically, the core attributes of the PCPCH model (i.e., access, accountability, comprehensive, continuity, coordination and integration, and patient and family centered care) include standards that key informants felt support the integration of mental health services into primary care settings. While it was clear from interviews that adoption of the PCPCH model does not necessarily mean that integration has also been achieved, the $\mathrm{PCPCH}$ program provided a starting point for clinics that had yet to begin to think about concepts such as integration and team-based care. Second, CCOs and the PCPCH model were linked in HB3650 (2011) and adoption of the model was tied to CCO incentive dollars, making the enrollment of Medicaid members in a PCPCH clinic a clear state priority. Thus, because the model was aligned with integration goals and incentivized by the state, it made sense from the CCO perspective to promote adoption of the model as a first step in the process of integrating mental health services into primary care settings.

Multi-payer system. Logistically, using Medicaid dollars to change the organization and delivery of care was perceived by many CCO key informants as extraordinarily difficult. Key informants, particularly those from CCO B which is responsible for a rural community, reported that a barrier in relying on Medicaid 
dollars to incentive practice change was that many clinics and providers exist and operate in a multi-payer system.

CCOs are expected to develop alternative payment methodologies (APM) to support new ways of organizing and delivering health care. This is particularly important for integration and greater cross-collaboration between systems because many of the services that are known to improve coordination have not traditionally been reimbursable. However, changes to the delivery of care cannot be isolated to only Medicaid patients. Any changes must be more broadly applied to all patients. There were concerns that additional payments for services delivered to Medicaid patients do not actually cover the full cost of practice change or care redesign.

The medical system at large is a multi-payer environment. And Medicaid is for the poorest patients and it's pretty challenging to steer practice when you're only one of several payers and you're not even covering their costs... (ID 0203)

We have a payment model but we're one payer so the clinics that have lots of our members, they can make this work, but if they don't have a lot of our members, it's really challenging to make it work. We definitely see in those clinics that have multiple payers, they'll tell us that they just don't see how it will work. (ID 0302)

\section{Administrative burden on mental health providers in primary care.}

CCO key informants also reported a lack of clarity around administrative oversight for mental health providers working in primary care clinics. The current regulations in place were originally designed for the community mental health system and intended to provide protections for individuals with SMI, who are frequently in a position of vulnerability when interfacing with providers. As more 
primary care clinics hire mental health providers, there is confusion regarding the applicability of some of the regulations as well as concerns about the burden on providers and clinics.

If I had to point to one specific thing...one specific barrier for integration and there's probably many things you could point to, but the burden of the regulations on the mental health and addictions folks and all the paperwork that they have to do is ridiculous... (ID 0302)

This was also an area that key informants noted where guidance from the state would be helpful. In addition, many felt that a state-wide discussion was necessary to create standards and regulations that are specific to mental health care in the context of primary care settings.

Findings specific to integrating primary care into mental health settings. The integration of care to address the needs of individuals with SMI was explicitly called out as a goal in the health Transformation Plans that each CCO was required to develop and submit to OHA. For many individuals with SMI, community mental health settings may be the only place they interact with the health care system, thus integration of primary care services in mental health settings may be necessary to improve outcomes and quality of care for this particular subpopulation. However, many CCO key informants reported that integration in mental health settings is extremely limited due to a number of barriers including perceived instability of the mental health system and lack of relevant incentive metrics. 
Instability of the mental health system. Noting that turnover among mental health providers is high and that clinics often have difficulty attracting and retaining the necessary workforce, CCO key informants expressed concern about the stability of the mental health system. Two key subthemes emerged from interview data and analysis of key CCO documents that help to explain this persisting challenge: 1) a historically underpaid workforce and 2) an increased administrative burden, relative to primary care.

Don't get me wrong; there is a lot of passion and I hope you capture that...there is a lot of passion in the specialty mental health system but they are underpaid and face an enormous amount of administrative oversight that primary care providers just don't deal with. (ID 0102)

The fact that people with graduate degrees are getting paid 40 thousand a year; that's not great. And if they can get a job in primary care or one of the hospitals or in one of the health plans, they're going to get a lot more money. So maintaining a workforce and paying them well enough to keep them around is a major problem...The level of pay for [mental health] providers is kind of atrocious. (ID 0103)

Key informants indicated that regulations regarding requirements for data reporting, certification, and licensing place significant administrative burden on mental health providers in both primary care and mental health settings. While many of these complex administrative requirements are intended to meet needs for accountability, they also require a significant amount of staff time and are costly. In the mental health system, where providers and staff have traditionally been paid less than in the primary care system, this administrative burden leads to less money spent and time allocated for direct care and programs. 
Low pay and increased administrative burden were connected with high turnover and issues with recruitment, retention, and distribution of the workforce, both of which contribute to access and quality issues that impact health outcomes. Thus, CCO key informants often reported that integration in mental health settings, while important, was unlikely to be achieved until the mental health system could be stabilized and issues of quality and access addressed.

Lack of relevant incentive metrics. Finally, while the metrics were seen as generally helpful in turning attention to the importance of integrating mental health services in primary care settings, according to one key informant the metrics selected and incentivized by $\mathrm{OHA}$ were not necessarily the ones that were most relevant and important for improving the health of the patient population seen by community mental health settings.

There are concerns about the metrics. Particularly that not all of them are going to be high priorities in a behavioral health clinic. So theoretically they could be doing great on the things that are really going to make an impact on a high acuity SPMI population but they're worried that they are still going to get dinged because we need them to perform well on this other measure. (ID 0301)

Certified Community Behavioral Health Clinic. In 2014, Congress passed the Protecting Access to Medicare Act (H.R. 4302), which includes provisions of the Excellence in Mental Health Act that authorized a two-part Certified Community Behavioral Health Clinic (CCBHC) Demonstration Program. The 2017-2019 pilot program is sponsored by the Substance Abuse and Mental Health Services Administration (SAMHSA) and is intended to establish a federal prospective payment methodology for the provision of comprehensive behavioral 
health services (SAMHSA, 2017). CCBHCs are required to provide services to anyone who seeks care, but their specific focus is on individuals with complex health care needs, such as those with serious mental illness, serious substance use disorders, and co-occurring mental illness, substance use disorder, or physical health disorders. CCBHCs are intended to increase access, stabilize people in crisis, and provide integrated treatment for those with the most serious, complex mental illnesses and addictions to emphasize recovery, wellness, and trauma-informed care (SAMHSA, 2017).

In the first phase, states submitted an application to receive a planning grant in order to solicit stakeholder input, establish prospective payment methods for reimbursable services, and submit an application for the demonstration program (SAMHSA, 2017). OHA applied for the planning grant because the $\mathrm{CCBHC}$ demonstration program is aligned with Oregon's larger health transformation initiatives and provides Oregon with the opportunity to further advance comprehensive behavioral health care for Oregonians (OHA, n.d.b). In October of 2015, 24 states, including Oregon, were awarded one year planning grants for the CCBHC demonstration program (SAMHSA, 2015).

In December of 2016, SAMHSA announced the eight states, of which Oregon was included, selected to participate in the pilot program (DHHS, 2016). Beginning in 2017, certified clinics in the eight states will receive an enhanced Medicaid payment rate based on their anticipated costs of providing an expanded array of addiction and mental health services, along with basic primary care 
screening and coordination with primary care (DHHS, 2016). Clinics in both CCO A and CCO B are participating in this pilot. Key informants noted that that they were not directly involved in the demonstration program. Interestingly, while $\mathrm{CCO} A$ and $\mathrm{CCO} B$ have clinics participating in the demonstration program, $\mathrm{CCO}$ A key informants only made passing references to the program, indicating that they were aware of it. However, key informants from CCO B and CCO C not only noted that they were aware of the demonstration program but also specified that they were watching it carefully.

We don't really have any bi-directional integration going on right now. The mental health program is participating in the CCBHC. It's more on the provider level...we are aware of it and we want to be in alignment with whatever they come out with. (ID 0204)

\section{CCO Case Specific Findings}

The three CCOs included in this study varied in terms of organizational structure, size, complexity, prior partnership history, and geographic service area. Table 4.1 presents a summary of $\mathrm{CCO}$ and community characteristics. For each $\mathrm{CCO}$, findings from the organization, professional, and clinic levels will be described in the following sections. This study used the RMIC's definitions of these integration types; these are briefly summarized below.

At the organization level, the RMIC defines integration processes as primarily focused on establishing organizational partnerships based on shared accountability and governance mechanisms to facilitate the delivery of comprehensive health care services to a defined population (Valentijn et al., 
2015). Key features of the RMIC's organizational integration level include interorganizational governance, trust, and inter-organizational strategy.

At the professional level, integration processes are defined by the RMIC as primarily building partnerships among health care professionals "based on a shared understanding of competences [sic], roles, responsibilities and accountability to deliver a comprehensive continuum of care" to a defined population" (Valentijn et al., 2015). The RMIC highlights three issues as central to this level: inter-professional education, agreements on interdisciplinary collaboration, and value creation for the professional.

At the clinical level, integration processes are defined as those processes that support "the coordination of person-focused care in a single process across time, place and discipline" (Valentijn et al., 2015). According to the RMIC, four key features are case management, continuity of care, interaction between professionals and clients, and individual multidisciplinary plans.

Table 4.1: CCO and Community Characteristics

\begin{tabular}{lccc}
\hline CCO & CCO A & CCO B & CCO C \\
\hline Number of OHP members & $>200,000$ & $>13,000$ & $\sim 30,000$ \\
\hline Coverage area & 3 counties & 2 counties & $\begin{array}{c}1 \\
\text { county }\end{array}$ \\
\hline Geographic region & Urban & Rural & $\begin{array}{c}\text { Small } \\
\text { urban }\end{array}$ \\
\hline History of organizational partnership & Yes & No & No
\end{tabular}




\section{Coordinated Care Organization A}

CCO A is the largest CCO in Oregon in terms of size, covered lives, and complexity. It is an umbrella organization, made up of multiple risk bearing partners, and serves a large, urban geographic region that includes three counties. It is responsible for more than 200,000 member lives and has 11 partners that share risk and responsibility for those lives. Of the eleven risk bearing partners, four are physical health plans, nine are dental plans, and the remaining three are the county health departments that focus on behavioral health.

Organizational integration. $\mathrm{CCO} A$ is responsible for a very diverse Medicaid population, some of whom are homeless, transient, and/or highly vulnerable. CCO A's founding partners have a prior history of collaboration. The governance board of $\mathrm{CCO} A$ includes a representative from each of the risk bearing partners (including primary care and mental health representatives) as well as other members who are elected and represent populations required by state statute as well as additional populations CCO A's founders felt necessary and important to include. Every board member's vote is equal; thus there is no weighting of votes based on status as a risk bearing entity, size of entity represented, or any other factor.

The structure of $\mathrm{CCO} A$ is unique as compared to other CCOs in Oregon. As an umbrella organization, it delegates risk to its partnering organizations. While CCO A has centralized many administrative functions including enrollment 
and customer service as well as data analysis to provide assessment and feedback, health transformation and integration is left mostly to the partners who bear the risk.

Key informants from CCO A reported that during the first few years of operation, they invested significant time and energy in building relationships with, and facilitating conversations among, their partners. Each of the partners operates as an organization independent of CCO A and thus has business interests that can at times be at odds with the collective interest of CCO A. Building relationships based on mutual trust, and developing and articulating a shared set of values, was critical for bringing together disparate organizations and balancing potential tension between $\mathrm{CCO}$ and partner interests. One of the ways CCO A attempted to achieve this was by identifying areas where there was alignment of interest.

But it can be a challenge at times to get all of the right people into the room and to be sure that you're speaking something that is of priority to them; finding a common denominator that applies to all of these organizations based on their different structure and approaches, really challenging. (ID 0102-S)

Because each partner has a different structure, different priorities, and different approaches to transforming care, it was easier to facilitate cross-partner collaboration when partners recognized synergy around shared priorities. In addition, key informants reported that CCO A's ability to leverage Medicaid dollars to incentivize change varied among the partners because Medicaid is only a small part of business for some business partners. 
Given that CCO A's delegates risk to its partners, its involvement with clinics and providers was limited and CCO A instead focused on facilitating conversations among the partners, developing a shared vision, ensuring that partners' health transformation activities are aligned with that vision, and maximizing learning among the partners and other stakeholders.

Our role is to nudge them along a little bit. When we discover that one entity is doing something awesome and really ground breaking, we want the others to learn from that and see if and how it may apply to them. So, we can do that through many forums but one might be a learning collaborative. (ID 0101)

The challenge of having partners with separate business interests, models, and approaches to care redesign is that alignment can be challenging; however, key informants also believed that the unique structure of CCO A potentially increased opportunities for shared learning and the spread of innovation.

We try to align the efforts of all of those organizations into something that looks like a shared goal, whether it comes to quality metrics, whether it comes to addressing particularly vulnerable populations, whether it comes to behavioral health integration, technology, that sort of stuff. Unlike many of the CCOs, we really have all these delegated relationships and we try to build alignment from that collective. That can have its pluses and minuses. It's hard to build alignment and yet the amount of experimenting or best practices that can bubble up in any of those organizations and then can be spread is really high compared to having a standalone or just one entity that tries to do one thing, one way. In that you either sink or swim. We have a lot of different organizations trying a lot of different things and so in a sense it creates a learning environment for different approaches. (ID 0102)

While bringing partners together to convene conversations is the main strategy that CCO A takes with the physical health risk accepting entities, CCO A's relationship with the three counties which serve as mental health risk accepting 
entities is slightly different. CCO A was more heavily involved in facilitating the transformation in the organization, financing, and delivery of care for behavioral health than it was for primary care. Key informants from CCO A reported that they worked closely with the counties for three years to create a regional behavioral health network in order to standardize administrative practices across the three counties.

The fact that these mental health organizations were limited to each of the counties and then each of those counties had their own approaches to doing this. [CCO A] spent so many hours...l'd say that there is where most of the heavy lifting happened; in aligning the administrative practices of the three counties so that they all have standard criteria for utilization reviews, standard authorization criteria, standard payment models so they're all paying the providers the same amount for the same type of service; that took about 3 years of bringing those counties together at least on a weekly basis to go through all these different elements to build up a system that looks much more common across the three counties so that if you move from one county to the next you don't have to start some new process. (ID 0102-S)

This finding will be discussed in more detail under clinical integration as regionalization was intended to address barriers mostly experienced by clinics and providers.

Professional Integration. Key informants from CCO A noted that professional culture can serve as barriers to integration, but that attitudes have begun to shift slightly since the development of CCOs. Increased opportunities for representatives from both mental health and primary care to engage in conversations have resulted in an improved understanding of the importance of mental health. 
Well now we have mental health and primary care people sitting at the same table and that's really important; it's key to getting people to change the way they view the world...you have to get them at the same table, you have to get them talking to each other. And they're sharing knowledge with each other that the other never knew before. And people are learning about mental health and the impact it has on health outcomes. That never happened before because of the silos. (ID 0104)

That said, while an increased understanding of the importance of mental health and a general willingness to consider integration is critical, there were also lingering concerns about the lack of behavioral health training for primary care providers.

Primary care physicians don't really get exposed to behavioral health in medical school. So they don't get it. And they're not sure how to handle it in the clinic...Well you know I think they're uncomfortable and some of them, they get the behaviorist in the clinic and they just leave those types of questions to the behaviorist. So they don't have to ask those tough questions. (ID 0105)

Another related concern is uncertainty regarding what team-based care looks like and the lack of role clarification for mental health providers working in primary care.

There is still some questions about the type of person you need in primary care. You want someone who understands behavioral health issues but at the same time is familiar with the fast-paced environment of primary care because it's a different environment than the one in a behavioral health clinic. And then you need to figure out what role are they going to play in primary care. Are they providing services directly to the patient or are they more of a consultant to the primary care provider. (ID 0101) 
Thus while the value of integrated care and recognition of the important role it can play in primary care have increased, there are still questions of what integrated care looks like in practice.

Community mental health workforce. Key informants from CCO A expressed concern about the perceived instability of the mental health workforce, citing low wages and excessive administrative burdens as primary causes for high turnover.

If we want people to stick around and become competent enough to know what they're doing, then we need to pay them enough in order for them to do that... (ID 0103)

In addition, while in general the value of whole-person, integrated care has increased, professional culture still serves as a large barrier in community mental health settings.

I think that [redacted] have been able to develop long standing relationships that have helped them come along a little bit to begin to even think about primary care but in general community mental health providers are primarily social service, not medical, so to medicalize them...that's a huge, huge barrier. I think that's the biggest barrier of all, probably even bigger than workforce. It's almost like there needs to be a reeducation to think about the whole person. (ID 0101)

In particular, one key informant reported that community mental health providers would find it challenging to offer primary care services because they have traditionally viewed themselves as social service providers.

Clinical integration. As a backbone organization, CCO A is not directly involved in efforts to facilitate integration; instead, that responsibility is delegated to its partners. While CCO A works to ensure that partner activities are aligned 
with the overall vision, direct involvement with clinics is limited; thus, key

informants' perspectives about integration were focused on the "big picture" more than on the details.

Primary care clinics. In general, key informants from CCO A felt that the $\mathrm{PCPCH}$ was helpful as a starting point for clinics new to integration and teambased care.

I think the PCPCH program has been helpful in that the tiers of the primary care home speak directly to behavioral health integration and they're going to be pushing on that more as they revamp that. (ID 0102)

There was a strong emphasis on recognizing that every clinic has a different capacity for integrated care and that expectations must be tempered with what can be realistically be accomplished. However, much of the work with clinics is left to the physical RAEs.

In addition, key informants from CCO A stressed the importance of flexibility for each partner. Rather than standardizing alternative payment models (APM), CCO A chose to focus on the creation of policies that promote and support the use of APMs while enabling individual partners to develop APMs that address their specific needs and fit within their existing business models.

So...It isn't possible for our partners to standardize everything... our partners have a range of organizational models and their own payment structures. I mean....we have full integration with capitation, closed delivery systems and, even a fee-for-service contracted network model. So we have this mix all along the continuum between two ends of the spectrum. The approach we've taken is that we create policies that promote APMs but they need to be free to develop APMs that work for their organization. (ID 0105) 
Thus, individual partners and their providers/clinics have piloted various APMs such as per member per month (PMPM) and incentive payments based on PCPCH Tier level to test effectiveness and sustainability before trying to implement APMs in a greater number of clinics. As of 2016, all four physical health plans in CCO A have implemented pay for performance as at least part of their total compensation strategy, and at least one of those plans has implemented an enhanced fee schedule to support behavioral health integration as well as team-based care and care management.

Mental health settings. As previously stated, CCO A worked more closely with the mental health risk accepting entities in driving the direction that the county health departments took in reorganization and financing of care. However, key informants report that integration of primary care services in mental health settings is not something that is currently being pursued regionwide. There are a few clinics in the region where CCO A operates that are participating in the state $\mathrm{CCBHC}$ demonstration program but those efforts are driven by individual clinics and not by CCO A.

We aren't really requesting that they [community mental health] integrate medical services at this time. I don't think we can do that right now. They aren't stable enough to take that on. We need to pay them more, [we need to] stabilize that system before we can ask that. (ID 0101)

Historically the counties that serve as the mental health RAEs have operated independently from each other, with each establishing its own administrative 
practices. This resulted in an enormous administrative burden on clinics and providers who could potentially end up responding to three different contracts.

CCO A has driven relationships between the counties... I think all that money being under the CCO forced the counties to talk to each other. The physical health plans haven't integrated nearly as much as the counties have. They all have very different models for how they do services but the counties were largely contracting with the same providers for the same types of services so it made a lot of sense to standardize.

While the primary driver for regionalizing the behavioral health network was to standardize administrative practices across the three counties, another driver was to improve the experience of care for patients.

[W]e don't see any reason to have three county-based organizations when there's one network. And it seems duplicative. And it seems contradictory to transformation and integration....Because specialty mental health is really a regional system of care. It's not a standalone. It's a regional service. People don't seek care because of the county they live in. They seek care because of the provider they want to go see. (ID 0106S)

Key informants reported that creating a regional system helped to support patient-centered care and ensure similar levels of resources were available in all three counties.

Part of regionalizing and coordinating the county mental health system involved implementing a standardized case rate (also known as an episode of care payment model) across all three counties. In addition to implementing a case rate for outpatient mental health services, CCO A key informants report that the mental health risk accepting entities also developed and implemented a 
systematic method to evaluate performance to help mental health providers make the transition to value-based payment.

They [county partners] use a case rate payment model for outpatient mental health services. And there's also a quality management performance fund incentive program that helped them make that transition to value-based payment. (ID 0105)

Case rate payments are intended to provide flexibility to the provider and client in determining the type, frequency and duration of services provided to ensure that mutually established treatment outcomes are met. The implementation of a case rate payment was not a strategy for facilitating integration specifically but rather a strategy to develop a value-based payment model to improve quality of care.

\section{Collaboration between primary care and community mental health.}

One final concern expressed by key informants was in regard to coordination between primary care and mental health settings. While integrating behavioral health care in primary care settings as well as primary care in mental health settings is ideal in order to meet the needs of individuals who will only be seen in one setting or the other, it is essential that the two systems be able to effectively communicate and collaborate about individuals who are referred to one setting to the other. While inoperability among electronic health records $(E H R)$ in different clinics or the lack of EHRs in some mental health settings were identified as barriers, key informants indicated that a lack of a common language was perhaps the largest barrier to overcome.

And the more interesting barrier...well, it's certainly a challenge to know who gets treatment in primary care with their embedded behavioral health specialist and to know who gets care in the 
specialty mental health system and we've never, certainly not as a state, but also not as a region, we've never had that discussion...I mean we're not going to draw a hard line in the sand; there is always going to be some gray area but generally speaking we've never outlined well, here's how that's going to be, here's who's going to get care from primary care with behavioral health support versus who's going to go into the community mental health system and I think we need to have that discussion. (ID 0101)

Summary of CCO A. CCO A is large and complex with multiple risk bearing partners. One of the primary challenges $\mathrm{CCO} A$ faces is in aligning partner interests with the overall interests of the CCO. Thus, according to key informants, the approach is often to align rather than standardize strategies. In addition, the structure of CCO A means that it has limited engagement with providers and clinics. Key informants report that the $\mathrm{PCPCH}$ model has been instrumental in helping clinics think about integration. Most of their primary care clinics have embedded behaviorist now, but there are lingering concerns regarding role clarification and uncertainty about the right skillset for a behaviorist working in primary care.

In contrast, integration in community mental health settings is extremely limited due to concerns about workforce capacity and stability. While there are efforts to increase access to primary care services, in particular there are a few clinics participating in the $\mathrm{CCHBC}$ demonstration program, these are not driven by $\mathrm{CCO} A$ but by individual clinics in its region. CCO A's efforts have instead focused on increasing access and quality by reducing administrative burden by facilitating the creation of a regional behavioral health network integrates the three county mental health departments. In addition, key informants expressed 
concerns that the lack of a common understanding about where individuals with varying risk and complexity of physical and mental health needs are best served; this common understanding was seen as essential to guide referral processes.

\section{Coordinated Care Organization B}

CCO B is relatively small in terms of size and covered lives; it serves a rural region that includes two counties. $\mathrm{CCO} B$ is an independent, not-for-profit health plan that has business lines in commercial, Medicaid, and Medicare insurance. It is responsible for slightly more than 13,000 member lives and is the sole risk bearing entity. However, CCO B delegated the responsibility of improving access and quality in community mental health to the local mental health authority. Serving as the fiscal, legal, and lead operating entity, CCO B is responsible for ensuring that health care transformation as directed by $\mathrm{OHA}$ and outlined in CCO B's Transformation Plan is carried out for members. In addition, the $\mathrm{CCO} B$ manages the contracts with downstream entities.

Within the first two years of establishment, CCO B struggled with some internal organizational challenges, primarily related to a significant turnover in staff. From 2014-2015, CCO B lost a number of its staff, and by mid-2015, a substantial portion of the staff members were entirely new. Thus, according to one key informant, CCO B had to essentially start over.

We're honestly in a restart mode, in the sense that I am new to the organization and I'm in a new position. I work for a behavioral director who is also new. That's a whole new structure. So pretty much everyone who's kind of key staff related to the CCO is new since about April...Our success in some of the stuff we've done has been limited by the difficulty finding people with project 
management skill. So that's put a limit on the CCO organizational side. (ID 0202-S)

Organizational integration. CCO B's geographic service area is a small, rural community; there are multiple small, private, primary care practices and the local community mental health program also serves as the local mental health authority. CCO B's governance board includes representatives from hospitals or other large health care organizations, FQHCs, mental health, community members, a member of the community advisory council, members from the clinical advisory panel, and members from each county as well as a representative from $\mathrm{CCO} B$; each member's vote holds equal weight. Whereas most other $\mathrm{CCO}$ and governance boards form a single corporate entity, CCO B is unique in that the governance board and the $\mathrm{CCO}$ are actually two separate entities with a joint management agreement in place. The board oversees the strategic plan and annual work plan, focuses on CCO performance metrics, is responsible for dispute resolution among stakeholders, and provides transparency and accountability to the community.

According to key informants from $\mathrm{CCO} B$, while the $\mathrm{CCO}$ bears full financial risk and responsibility, the unique structure of $\mathrm{CCO} B$ and its governing board was intentionally designed to maximize local control and priorities as well as establish a foundation of trust.

The CCO has a vote on the governance board, but the governance board is the one that directs the policy and even some of the financial decisions. There are some shared gains; money is split between the $\mathrm{CCO}$ and the governance board if there is a surplus. And then the governance board makes decisions about how to 
spend those monies in various ways. It is unique. It really gets the community involved at a lot of levels. (ID 0203)

Key informants from CCO B emphasized the importance of building and maintaining trust with community partners; thus some of CCO B's board meetings are open to the public to enhance transparency. According to one key informant, it is not unusual for local providers and other health care stakeholders to attend the meetings on a regular basis to stay informed. In addition to holding open and transparent meetings, CCO B utilizes listening sessions to gain stakeholder input, and relies on the governance board to engage providers and other relevant health care stakeholders. The emphasis on community control and local priorities has led to a fairly inclusive and collaborative decision-making process that can be slow; however, key informants report that it also facilitates greater community commitment and buy-in.

There's a high degree of local ownership and local control. And they look to the plan to help them but to not be the answer, which I think is very healthy. That leads them often to confronting, sometimes, that the things that we can finance are primarily Medicaid things. And many of the solutions that they seek are multilayered, multisystem solutions, communitywide solutions. So sometimes the things that they want to achieve, we can only be part of the solution and not all of the solution. But it's a huge strength that there's a high degree of ownership. (ID 0201)

Interviews with key informants from CCO B indicate that the CCO itself has focused mostly on developing payment models that are financially sustainable and support transformation of care. As a health plan with lines of business in Medicaid, Medicare, and commercial insurance, CCO B is well acquainted with the realities of a multi-payer health care system. Thus, its efforts to facilitate 
integration have focused on developing payment models that will help ease providers into changing their way of doing business.

Professional integration. According to key informants from CCO B, the strong emphasis on community engagement with open and transparent meetings and locally driven health transformation has resulted in a dramatic increase in dialogue among health care stakeholders, including representatives from primary care and mental health.

Just getting people together at the same table to discuss integration and what that means and making sure everybody is on the same page. This is a complete paradigm shift in peoples' thinking. (ID 0204)

However, key informants from CCO B reported that there are still some challenges associated with attracting and retaining the appropriate workforce. Part of this difficulty is because CCO B's geographic service area is rural; rural regions often face additional challenges in ensuring an adequate health workforce (MacDowell, Glasser, Fitts, Nielson, \& Hunsaker; 2010). Another part of the difficulty in attracting and retaining the appropriate workforce was related to the lack of widespread education and training opportunities for mental health providers working in primary care settings.

One issue is there just isn't enough of them [licensed professionals] and then another is that many in this workforce aren't trained or prepared to work in a primary care setting because it requires a very different skill set and a different ability to adapt to a much different workflow. It's much quicker, you know so not 50 minutes, and they have to be able to handle being pulled out of the room or to being interrupted. (ID 0201) 
In addition, one key informant observed that since the cultures of mental health and primary care are fundamentally different, there was a lot uncertainty understanding what skills and characteristics are necessary for mental health providers working in primary care settings.

What are the right training needs for someone to...What is the right type of person do you need in a primary care clinic, because the cultures are so different, and different from specialty behavioral healthcare or alcohol and drug treatment. What are the qualities that you need in a behavioral health consultant in those areas...There's no real training program in school for this right now. So it has to be on the job training. (ID 0206-S)

Community mental health workforce. Key informants from CCO B also expressed concern about the potential impact that the drive to integrate mental health services in primary care settings may have on the community mental health system workforce.

Well, first of all, there is no workforce. I mean, there really isn't, especially in rural areas. Particularly because of this expansion as well as these grants that came out. Everybody started hiring at the same time. So there's a lot of competition for the folks that are out there. So there's a lot of green people coming right out of school that people are competing for. (ID 0306-S)

With primary care seeking mental health providers and likely paying them better...there is some concern that it may have an impact on the skilled workforce in specialty settings. (ID 0204)

Health transformation has resulted in high demands for workers with mental health knowledge. One of the ways that the region has attempted to deal with this issue is through an agreement between the mental health authority and the local FQHC to work together. 
The community mental health program in our more rural areas are actually providing staff in some of the FQHCs. So in [redacted] the mental health program has put a full time FTE in the FQHC that is located there and they provide some behavioral health consultation to the primary care providers in the clinic and help them manage their patients. (ID 0205)

Clinical integration. CCO B places a strong emphasis on understanding the multi-payer reality of the providers in the region and on developing payment models that not only support integration but are also financially sustainable.

Primary care clinics. While key informants reported that the $\mathrm{PCPCH}$ offers a useful model for integrating care in primary care settings, they also indicated that it was challenging to generate interest among providers in the $\mathrm{PCPCH}$ recognition process. $\mathrm{CCO} B$ partnered with its governance board to ensure that clinics were aware of the PCPCH standards, requirements, and opportunities as well as providing clinical and technical support to assist small, private practices in seeking and maintaining $\mathrm{PCPCH}$ recognition. In addition, CCO B developed and executed a strategy to increase the PMPM capitation rates to enable primary care providers to invest in a number of activities, including PCPCH adoption. This combination of outreach, technical assistance, and investment enabled CCO B to nearly double the percentage of its members enrolled in a PCPCH in one year.

In addition to increasing extent of $\mathrm{PCPCH}$ recognition, $\mathrm{CCO} B$ used some of its transformation funds to support several projects intended to facilitate integrated care in primary care settings. Some of these projects included the placement of behavioral health providers into primary care settings and a pilot 
project to develop a better understanding of how to best support the integration of primary care and mental health.

Most of the focus and work has been integrating behavioral health consultant services into primary care settings. We used some of our transformation fund dollars to do some practice facilitation. I think it helped us understand the dynamics around sort of what's currently working, what hasn't really worked, what the barriers are. There hasn't actually been a significant change in what any clinics are offering. But it does feel like we're a little closer to getting there. (ID 0202)

Specifically the pilot project entailed engaging four primary care clinics and the local mental health authority in the development of a framework for the integration of behavioral health. Clinics were provided technical assistance as they identified or developed and refined models of integration. Consultants assisted each of the sites in gathering information about their current standard of care, analyzing workflow processes, developing an idealized vision of integrated care, and estimating total annual expenditure of integration. In addition, they provided support, resources, and guidance to help the clinics develop the capability and capacity to collect, track, and report measures of quality and performance.

Parallel to these activities, CCO B also worked on understanding provider perspectives on APMs and developing payment models to facilitate adoption of $\mathrm{PCPCH}$. In early 2012, CCO B signed a contract with a provider organization that represents a large portion of the providers in the CCO B's region. Key elements of payment reform to support integrated care in the contract included capitated monthly payments, risk-adjusted based on OHP eligibility categories. 
$\mathrm{CCO} \mathrm{B}$ also provided additional funding for $\mathrm{PCPCH}$ enhanced care models that met cost and quality outcomes. To obtain feedback on APMs, CCO B initiated listening sessions with community stakeholders.

We held a listening session. Providers told us that changing their payment models and their care delivery for only one line of business is not practical, especially in rural health care environments...they understood that payment reform is necessary but they need transitional methods to give them time to adjust. (ID 0205)

One of the key challenges $\mathrm{CCO} B$ faced is that most of its providers operate in a multi-payer system. While this is a characteristic of the health care system at large, it is complicated in rural regions that have a lot of small, private practices. For many of these clinics, Medicaid alone cannot cover the cost of changing the way they deliver care and thus participation in health transformation is often perceived as financially unfeasible.

Most of our CCO providers are really functioning in a multi-payer environment, where Medicaid is twenty, thirty, maybe forty percent of their revenue stream. But it can't make a model fly. We haven't been able to make a model fly without understanding their multipayer realities... (ID 0201-S)

In 2015, CCO B implemented a payment model in one of the local clinics; the payment model enabled the clinic to hire additional staff, specifically behavioral health consultants, to work with primary care providers. These behavioral health consultants work alongside primary care providers as part of a care team to provide whole-person care to individuals.

We have a model with [redacted]; it's kind of an alternative payment model, where we kind of estimate the number of members that are receiving services in the clinic, per month, and then pay a figure 
based on that. It's not a pure PMPM model. It's kind of a funky model. But it does allow for them to be doing some integration with behavioral health consultants and psychiatry. But we're looking, I think down the road, to beef up that as well and to really develop an integrative collaborative with community stakeholders who are interested in that and to look at how we can make that payment system and model more robust and spread integration beyond just that one clinic. (ID 0203)

In addition, CCO B implemented a new billing arrangement to facilitate integration by reducing administrative oversight to primary care clinics providing mental health services. In order to qualify, the clinic is asked to measure the level of integration using the Integrated Practice Assessment Tool (IPAT) available on the Substance Abuse Mental Health Services Administration and Health Resources and Services Administration's Center for Integrated Solutions (Waxmonsky, Auxier, Heath, \& Wise Romero, 2014). Clinics that score above a certain level on the IPAT are no longer required to seek preauthorization from CCO B to provide selected mental health services.

While key informants from CCO B acknowledge that current incentives are not perfectly developed and not necessarily true APMs, they believe they are stepping stones that enable the clinics to move forward with integration as $\mathrm{CCO}$ B now works on more nuanced payment models and in particular considers how to create a model that can support clinics' transformation efforts in a multi-payer system.

Mental health settings. Integration of primary care services in the local mental health program was nonexistent for the first three years of CCO B's operation and even now appears to be extremely limited. While the mental 
health authority was a participant in the early pilot project to develop a framework for primary care and mental health integration and received consultation services alongside the four primary care practices, one of the early learnings was that integration in mental health settings is incredibly challenging. CCO B key informants expressed concerns about workforce capacity; they were particularly concerned that because primary care was now seeking mental health providers that there would be a negative impact on the number of qualified workforce available to work in community mental health. The mental health authority collaborates with an FQHC by placing their own mental health providers in the FQHC, but key informants suggested that efforts to experiment with the model of care in the community mental health program itself have been slow. In order to improve access and quality, $\mathrm{CCO} B$ and the county mental health program have a sub-capitated arrangement where a portion of the sub-capitated rates are paid if the community mental health programs meets certain $\mathrm{CCO}$ requirements related to quality and access metrics.

A full on sub-capitation to mental health, that's typically done, historically, without any quality measures or any expectations for the sort of volume of service or wait times or other parameters. So there aren't incentives built into that payment structure for the people getting the money to really provide an adequate amount of care that is a really high quality. There's obviously an incentive not to over-treat, which is good. But potentially, an incentive to undertreat because the money is gone either way...the [mental health authority] is showing more willingness to engage and think about different ways of doing business or diversifying the network of providers. (ID 0202-S) 
A goal for 2017 for $\mathrm{CCO} B$ is to ensure the integration of primary care services into at least one of the mental health authority's programs. In preparation for that goal, the mental health authority hired a mental health nurse practitioner to work with members with SMI and participated in the state-wide CCHBC Demonstration Program.

We're just at the beginning process of that in the FQHC up there that is looking to partner with the community mental health program to develop some reverse integration. (ID 0203)

The local FQHC and the local mental health authority already partner to provide integrated care in the FQHC and are working together to determine how this would be organized and what would be required to place a primary care provider from the FQHC into the mental health program. However, these activities are focused on the provider level and thus CCO B has limited involvement.

We're not really developing a lot in bi-directional integration or reverse integration because we're kind of waiting for that to fall out and see where the chips fall; and there's such an effort underway particularly in specialty behavioral health to meet those requirements that it's kind of taken on a life of its own so it's kind of like...now is not the time let's do this cool reverse integration clinic because that conversation has to take place within the conversation of what a CCBHC is going to be and what it's going to deliver. The overall $\mathrm{CCBHC}$ issue is kind of dominating that conversation. (ID 0201)

In addition, key informants indicate that $\mathrm{CCO} B$ is waiting to see the results of the $\mathrm{CCBHC}$ demonstration to ensure that any future $\mathrm{CCO}$ efforts are aligned with the CCBHC standards.

Collaboration between primary care and community mental health.

As previously mentioned, there is some existing cross-collaboration between 
primary care and community mental health. Faced with workforce capacity concerns and pressure to integrate care, the local FQHC and the community mental health program partnered to place a mental health provider in the FQHC. However, key informants indicated that given that integration in primary care settings is a relatively new phenomenon, there is not a lot of guidance around when and how to make a referral.

There is not a common understanding about what should be referred and what should be handled in primary care. It does come up in the forums that we've set up for these types of discussion. Some primary care clinics are way more robust in terms of the kinds of things they can manage than others so it's important to maintain [integration] as an aspirational goal. As clinics evolve their capabilities, we want to be able to say here's where we want them to be, but at any point if anyone is thinking about making a referral, here is the kind of engagement and clinical pathway that we recommend. (ID 0203)

Summary of CCO B. CCO B is responsible for a rural geographic region and has taken a strong community-driven approach to health transformation built upon transparency and trust. Key informants report that while most of its primary care clinics are recognized as PCPCHs, delivery of care has not changed significantly. Two of the largest primary care clinics have hired behaviorists. However, this is far more challenging for the majority of CCO B's small, private clinics. Key informants reported that changing a clinic's delivery of care for a smaller subset of the patient panel is not financially sustainable. Thus, CCO B has focused on the development of transitional payment models to support clinics as they work on changing their business models. In addition, there are 
concerns about workforce recruitment and retention and role clarification for mental health providers working in primary care settings.

Integration in community mental health settings is essentially nonexistent. However, the local community mental health program participated in the CCBHC planning grant and is participating in the demonstration program. CCO B is waiting to see how the $\mathrm{CCBHC}$ develops in order to ensure any future efforts are aligned with the CCBHC standards and model. Interestingly, cross-collaboration between primary care and mental health occurs both informally and formally, but these efforts appear to only impact the provision of care in primary care settings. Finally, key informants from CCO B expressed a desire to build standardized clinical pathways and recommendations to guide referral processes between primary care and community mental health.

\section{Coordinated Care Organization C}

CCO C is a wholly owned limited liability corporation of a physical health

plan, and serves a small, urban geographic region that covers one county. CCO $\mathrm{C}$ has an operating agreement with the physical health plan and uses its administrative, claims processing, medical management, and customer service infrastructure. Responsible for roughly 30,000 lives, CCO C has seven risk bearing partners. One of the partners is a physical health plan which owns CCO $\mathrm{C}$ and is responsible for medical as well as residential and outpatient addiction services, four are dental health plans, one is a nonemergency medical 
transportation company, and the last is the county which manages and provides mental health services.

Organizational integration. The governance board is made up of representatives from the health plan which owns $\mathrm{CCO} C$ as well as from local hospital systems, FQHC clinics, addiction treatment service providers, and the county health and human services department in addition to a private practitioner and two members of the Community Advisory Council. Each board member including the health plan that owns $\mathrm{CCO} C$ has an equal voting share to ensure equal participation.

Key informants from $\mathrm{CCO} C$ reported that because the $\mathrm{CCO}$ was a new organization with no prior history of collaboration among partners, building trust was critical to efforts to develop the necessary relationships in the community in order to work towards health transformation and facilitate the integration of primary care and mental health. Central to CCO C's efforts to build and maintain this trust was the recognition that organizations in the community have more expertise in their respective areas of health care than the CCO.

We continue to have a strategy within [CCO C] that this isn't all our world and our work. It's really about us doing these efforts in partnership with all the other organizations on the ground and really supporting and building their capacities. (ID 0305)

Originally CCO C intended to contract with a regional behavioral health organization; however, that organization dissolved and the county stepped in to assume that risk. While experienced as a provider of mental health services, the county had no prior experience with managing risk. The county is also a provider 
of mental health services but holds subcontracts with other mental health providers in the region. As of January 2017, CCO C is combining the mental health risk with the physical health risk rather than delegating it to the county. According to key informants, this decision should enable CCO $\mathrm{C}$ to expand its mental health network.

Of the three CCOs, CCO C has the most direct involvement with providers and clinics. CCO C opted to focus on building clinic and provider capacity first as opposed to developing alternative payment models. Its strong consultation model is based on the assumption that clinics and providers have to learn what integration is and how to implement it and that alternative payment models simply support those efforts rather than drive them. CCO C places specialists into primary care clinics to work with primary care providers and behaviorists and provide support as they figure out their respective roles and responsibilities and learn how to work together as a team.

Professional integration. Similar to CCO A and CCO B, the mental health and primary care systems in CCO C have historically operated in silos. However, broader health transformation has increased opportunities for health care stakeholders in the community to learn from each other. Key informants have identified this as critical for cross-collaboration.

[Name redacted] from county mental health came and talked about their programs. Just, here's who we are and here's what we do. And here's a bunch of new stuff that's really quite innovative. And we have to break down silos because we need to be able to communicate about financials, claims, clinical data...setting those kind of relationships and conversations up is really important. So 
education about who and what mental health is and does. (ID 0301)

In addition, provider champions have played an important role in advocating integration with other providers.

Well and there is resistance but once they do it, once the clinic integrates mental health services, they don't want to go back because they see the outcomes. And then the clinics that already have done it, the physicians have championed it; they're going out and meeting with their colleagues...So yes I think there is a growing awareness that mental health issues are important, that integration is important. (ID 0304)

However, as in many other communities, CCO C has found that the general lack of knowledge about mental health is a continuing challenge.

What we realized at the provider level is there just needs to be a lot more education. The physical health world just does not know the mental health world; what's offered, how to get to it, what patients need. (ID 0301)

Primary concerns about the workforce included capacity (i.e., not having enough workforce to fill the need) and competency (i.e., not having a workforce trained to do this type of work).

The biggest barrier is workforce that would be 1, 2, 3, if I had my choice. It's incredibly challenging for people to do this work when they don't have the staff and then you have to talk about do they have experienced staff. This is a new work space for behaviorists and so they have to have the right understanding and cultural fit and etcetera. (ID 0302)

These workforce concerns regarding competency are what led CCO C to adopt a consultation model to help build capacity within clinics so that primary care providers could learn how to work with mental health providers. 
Clinical integration. In contrast to the first two CCOs, CCO C is actually directly involved in efforts to facilitate integration. This level of involvement has lead it to have a greater understanding of clinical operations and the challenges that clinics encounter when trying to provide team-based, integrated care.

Primary care clinics. In general, CCO key informants felt that the $\mathrm{PCPCH}$ program was helpful in introducing basic concepts to clinics new to integration.

They've really evolved the PCPCH model. And I think it's been quite valuable. If you actually get in and do some of this work where you're actually trying to think of your patient population as a patient population and not a panel. And you're able to look towards outcomes and move your patient population towards a new goal, that is the essence and the foundation of what the real need is around a patient centered medical home... It brings such valuable concepts, especially in terms of team-based care, and bringing mental health into a primary care office that is not used to that world... It's a way of introducing some basic topics about quality improvement, team-based care that are just, honestly, very, very new to some of these folks. (ID 0302)

CCO C had a number of small, private practices in their community and found it challenging to encourage those clinics to adopt the PCPCH model and become certified. Two issues were apparent in efforts to increase the percentage of members assigned to a PCPCH: (1) clinic outreach, advocacy, and marketing, and (2) guidance and support for clinics interested in achieving recognition.

Recognizing that small, private practices may find meeting the $\mathrm{PCPCH}$ criteria challenging, CCO C created a primary care administrator group to develop unique strategies and incentives. A practice coach was hired to provide 
targeted support and facilitate a PCPCH learning collaborative as well as a PCPCH-based care management collaborative for clinics interested in pursuing $\mathrm{PCPCH}$ recognition. Despite this work, $\mathrm{CCO} \mathrm{C}$ found it difficult to generate interest.

In order to address this issue, $\mathrm{CCO} \mathrm{C}$ assembled information about the $\mathrm{PCPCH}$ program and began to engage the clinical advisory panel in a discussion about the costs and benefits of PCPCH implementation. It also sent CCO staff to key practices to generate interest. Highlighting the $\mathrm{PCPCH}$ model as an opportunity to enhance learning and receive practice support, CCO C directly advocated the model within its community. However, CCO C quickly discovered that its practices were struggling with how to operationalize PCPCH standards. It shifted its efforts to providing technical assistance and practice consultation by hiring staff and placing them in primary care practices to provide direct support to providers and staff.

We hired staff who are really helping in the clinics, helping the providers and staff think through primary care, think through behavioral integration in a much deeper way. That tends to be about care coordination. (ID 0305-S)

CCO key informants reported that these clinic support staff help the providers think through what team-based care looks like in their clinic as well as problem solve and develop models of integrated care.

Our alternate payment models have not...We're going a different road than some others where we're not just saying here's our new payment model. You have to do so many ticks. We have the fundamental belief that folks need some help and some support and some basic capacity building before they're ready to do any 
kind of significant...yeah. So as part of that, we released significant funds this year to help our primary care clinics, our main ones, build up their behavioral health capacity. So it's not that they're getting paid in a whole new model, but we did ask them to keep track of all the behavioral health visits they did and report those back to us. (ID 0302)

While CCO $\mathrm{C}$ recognizes that APMs are important to support integrated care, its approach was to build capacity first. Thus, early efforts centered on developing an APM that would guide the allocation of shared savings, evaluating the current payment structures, and identifying the potential payment models that would transition their providers from fee for service. CCO $\mathrm{C}$ now has a PMPM tiered quality measure based payment that will be introduced to select clinics that have successfully built the capacity to support integrated care.

That [specialist support] was going on for a full year and then we introduced our alternative payment programs. There were two tracks, the first track is about creating a capacity building space for a year and in that space we really set some criteria that helped them get ready for track 2 , and track 2 is a PMPM tiered quality measure based payment for behavioral health integration so it has measures of access and open access and ratio of behavioral health providers to primary care providers and just making sure that team based care is happening, making sure that behaviorists are included in huddles. (ID 0302)

Mental health settings. Having delegated the risk and management for mental health to the county, CCO C focused mostly on the integration of care in primary care settings. The county worked on developing the capacity and expertise to manage the risk as well as expanding access and enhancing the quality of the county mental health system.

The focus was primarily on making sure that primary care behavioral integration was working and then county was working 
with their providers in making sure there was access and the appropriate programs. So they worked hard to make sure that there is access and capacity in the community. (ID 0301)

CCO key informants report that integration in mental health settings is limited and that efforts in community mental health have focused on improving access. However, CCO C, in partnership with multiple community stakeholders including a local FQHC and the county, helped to develop and fund the creation of a PCPCH clinic and placed it in the county building in close proximity to the community mental health program.

It sits in the new County building, which is where mental health services sit as well. So it's co-located with our mental health...It's an FQHC site. And it's a partnership at the administrative level. It's a partnership between us and [redacted] who are supporting it, the [county name] and both of the main A\&D providers. And the goal is to provide primary care for those with SPMI or really acute addiction diagnoses. (ID 0305-S)

Housed in the county building, the co-location of the clinic with community mental health enables primary care providers to maximize on warm handoffs and walk patients down the hall to mental health providers. Warm handoffs refers to a referral practice where the primary care provider introduces patients to the mental health providers in real-time (Davis et al., 2015). It is thought to increase patient satisfaction and trust as well as improve care coordination (Davis et al., 2015).

In addition, when the $\mathrm{CCHBC}$ planning grant was first announced, $\mathrm{CCO} \mathrm{C}$ considered applying for the opportunity; however, key informants report that CCO C could not figure out how to make the payment model it developed fit with the 
behavioral health home guidelines. While CCO C opted out of applying for the $\mathrm{CCBHC}$ demonstration pilot, a key informant indicated that the CCO was closely monitoring its development.

We're watching very closely the CCBHC work happening at the federal and state level and we really want to be open to collaboration to see what's the sustainable funding in that space, what's the outcomes that are expected in that space, what's the model look like, so while we decided not to do, very consciously, not to do the behavioral health home, we are very much wanting to line up in that space... (ID 0302)

Payment is currently sub-capitated for mental health, but there are plans to incorporate mental health services into CCO C's APMs. The first step in this process is combining the funding streams for physical and mental health. In order to prepare for this transition, $\mathrm{CCO} \mathrm{C}$ has begun building capacity (i.e., expanding its mental health network) and strategizing around what integrated care means for $\mathrm{CCO} C$ and its members. In particular, one key informant mentioned that pooling funding will enable $\mathrm{CCO} C$ and its partners to develop their own version of behavioral health homes to ensure comprehensive services are available to individuals with serious mental illness.

Finally, bringing the funding together will also enable $\mathrm{CCO} C$ to generate a better understanding of its community's health, utilization, and cost of care.

We're going to have the opportunity now to capture claims now across physical health, addiction, and mental health and when you start looking at Triple Aim and that whole reason why we get a global budget, it's really about where we can improve outcomes, improve patient experience, and decrease costs. By bringing the mental health in, we'll be closer to being able to track that data around cost, utilization, and outcomes in much more in-depth way than we were able to before. And that's going to take some time to 
build that, we have to build the experience and the claims database, so that will take some time. (ID 0302)

Collaboration between primary care and community mental health.

In terms of cross-collaboration between primary care and community mental health, the community itself appears to have developed values centered on ensuring that people get the care they need no matter where they engage the system.

Well and you know in most communities, it's the primary care provider that does the referrals but all of our programs in the community are able to do that. So if someone is working with an individual in one of those programs and finds out that they haven't seen a primary care doctor in years, they can help connect that person so they can get the care they need. (ID 0301)

Thus, multiple programs throughout the community have informal and formal policies in place that enable providers and social workers to help ensure that individuals are connected to mental health and primary care services. This is facilitated by the relatively small size of the community; all health related organizations are within walking distance of each other.

Summary of CCO C. CCO C is responsible for a small, urban geographic region. Key informants reported that the $\mathrm{PCPCH}$ model played an important role in facilitating integration of mental health in primary care settings. They noted that after conducting significant outreach and advocacy to generate interest in the PCPCH model, they discovered that the clinics were having trouble operationalizing some of the standards such as team-based care. In particular, there was confusion regarding role clarification and understanding how to include 
mental health providers as part of the primary care team. To address these issues, the CCO placed consultants in the clinics to provide direct one on one support to help clinics problem-solve and to guide clinicians as they figured out what integration means and looks like in practice.

Integration in community mental health settings is limited; however, the $\mathrm{CCO}$ and community stakeholders partnered together and set up an advanced $\mathrm{PCPCH}$ in the same building as the county mental health program for individuals with SMI and/or substance use disorders. Key informants from CCO C reported that the community itself values the idea that there is no wrong door for individuals. Thus, there are mechanisms in place to connect individuals to primary care, mental health, and other health or social service needs. As of 2017, CCO C has integrated the funding streams for both primary and mental health care.

\section{Summary of Integration Level Findings}

Building upon the description of findings for each $\mathrm{CCO}$, each level of integration will now be briefly summarized with attention to which features of the RMIC framework were manifested during data analysis and the identification of key themes for each level.

Overall, the degree to which key informants from CCOs discussed different levels appeared to have been shaped by the level of engagement the CCOs had at the different levels. CCO A had extremely limited engagement at the professional and clinical levels. CCO B similarly had limited engagement at 
the professional and clinical levels, primarily engaging with providers and clinics around the issue of alternative payment models. In contrast CCO C appeared the most engaged at the professional and clinical levels.

To facilitate a synthesis of the findings, key findings are presented in the tables below. Numbers in the tables are used to contextualize the data. A score of 0 means that a particular feature was not mentioned; a score of 1 means that a feature was alluded to; and, a score of 2 means that a feature was directly discussed. In addition, pluses and minuses refer to whether this feature was noted to be a barrier/not helpful or facilitator/helpful. A plus and minus means that a feature was both a barrier/not helpful and a facilitator/helpful.

System integration. At the system level, all three key features of the RMIC manifested in the data: regulatory frameworks, political/social environment, and financial factors. Table 4.2 summarizes the key features and findings related to system integration.

At the system level, alignment of regulatory frameworks, the political/social environment, and financial factors for integrating mental health services into primary care settings were all scored as $2+$ because CCO key informants both directly discussed these features and for the most part reported on these features as being mostly helpful in facilitating integration. Conversely, alignment of regulatory frameworks and financial factors for integration of primary care services into mental health settings were scored as 2-; CCO key informants directly discussed these features but reported that for the most part these were 
barriers to integrating care. Finally, political/social environment for integrating primary care services into mental health settings was scored as $1 \pm$ as CCO key informants alluded to but did not directly discuss this. However, it appeared that while in general the idea of integrating primary care into mental health was accepted as ideal, there was still some resistance to actually trying to achieve integration in mental health settings.

Table 4.2: RMIC System Integration, by CCO

\begin{tabular}{lllllll}
\hline & \multicolumn{2}{c}{ CCO A } & \multicolumn{2}{c}{ CCO B } & \multicolumn{2}{c}{ CCO C } \\
\hline & PC & MH & PC & MH & PC & MH \\
\hline $\begin{array}{l}\text { System Integration } \\
\begin{array}{l}\text { Alignment of regulatory } \\
\text { frameworks }\end{array}\end{array}$ & $2+$ & $2-$ & $2+$ & $2-$ & $2+$ & $2-$ \\
\hline $\begin{array}{l}\text { Political/social } \\
\text { environment }\end{array}$ & $2+$ & $1 \pm$ & $2+$ & $1 \pm$ & $2+$ & $1 \pm$ \\
\hline Financial & $2+$ & $2-$ & $2+$ & $2-$ & $2+$ & $2-$ \\
\hline
\end{tabular}

$0=$ not mentioned, $1=$ alluded to, $2=$ directly discussed

$+=$ helpful, $-=$ not helpful, $\pm=$ mixed

Regulatory Frameworks. Key informants commented on a number of regulations such as data reporting requirements, certification and licensing, and contracts that place significant administrative burden on mental health providers in both primary care and mental health settings. While many of these complex administrative requirements are intended to meet needs for accountability, they also require a significant amount of staff time. In the mental health system, where providers and staff have traditionally been paid less than in the primary care system, this administrative burden leads to less money and time spent for direct care and programs. In addition, key informants reported that licensing as well as reporting and billing requirements also negatively impacted mental health 
providers working in primary care settings. While recognizing the importance of accountability, they were concerned that these rules were created for providers working in community mental health settings and questioned the applicability in primary care settings.

Political/Social Environment. Visionary leadership played an important role in facilitating state-wide buy-in and commitment. Oregon's health transformation effort was framed as an attempt to redesign the organization, delivery, and financing of health care to achieve better health at lower costs, in pursuit of the Triple Aim. Within this larger health transformation narrative, the integration of primary care and mental health was highlighted by $\mathrm{OHA}$ as an important component for improving the health care delivery system.

However, key informants were concerned about a perceived lack of guidance on administrative rules regarding mental health providers in primary care settings and the development of APMs that are actuarially sound. In addition, several key informants felt that broader issues that impact the mental health workforce, such as low pay and administrative burden, should be tackled by OHA or through a state-wide discussion to develop strategies for addressing these particular problems.

Financial factors. A number of issues related to financial factors emerged including incentive metrics and multi-payer systems.

Incentive metrics were helpful in driving attention to the importance of mental health in primary care. By tying dollars to achieving benchmarks on 
certain measures, CCOs had leverage to encourage clinics to focus on certain diagnoses. In addition, OHA's decision to include measures that touched on mental health sent a broader message that mental health concerns were important. That said, there are a limited set of defined measures that have anything to do with mental health, even fewer that apply to integration in primary care settings, and none that apply to integration in mental health settings.

Multi-payer systems. CCOs were tasked with developing alternative payment models to support practice change and care redesign. However, key informants report that for many primary care practices, relying on Medicaid is a limitation in a multi-payer environment because those dollars are only a small percentage of their overall business.

Organization integration. At the organization level, all three key features of the RMIC manifested in the data: inter-organizational governance, strategy, and trust. At the organization level, CCO A and CCO B scored the same for

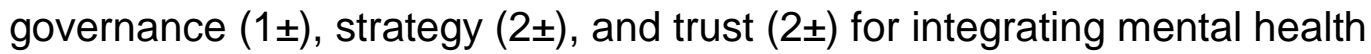
services into primary care settings meaning that key informants from both CCOs alluded to governance but did not directly discuss it. However, key informants from $\mathrm{CCO} A$ and $\mathrm{CCO} B$ did directly address both strategy and trust. The findings indicate that all three key features for both CCOs were mixed in terms of whether or not these features were in place in a way that was helpful for facilitating the integration of care in primary care settings. Conversely, CCO C scored a $1+$ for governance, a $2+$ for strategy, and a $2+$ for trust, meaning that 
key informants alluded to governance and spoke directly to trust and strategy; the data suggest these features were in place in a way that was helpful for facilitating integration of mental health services into primary care settings.

In terms of integrating primary care services into mental health settings, CCO A and CCO B again scored the same across governance (1士), strategy (2-),

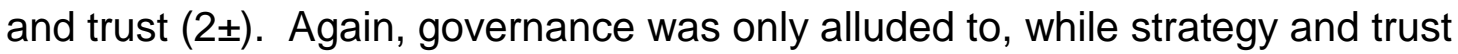
were spoken about more directly. Governance and trust were scored as mixed in terms of whether these features were in place in a way that was helpful while strategy was scored as not helpful for facilitating the integration of care in mental health settings. CCO C scored a $1+$ for governance, a $2 \pm$ for strategy, and a $2+$ for trust, indicating key informants from $\mathrm{CCO} C$ alluded to governance and directly addressed strategy and trust. In addition, data indicated that governance and trust were in place in a way that was helpful for facilitating integrating of primary care services into mental health settings, while strategy was scored as mixed. Strategy received a mixed score for being helpful because while CCO C found a way to bring primary care to individuals with SMI and/or SUD, CCO C's workaround was limited to the community mental health program. Table 4.3 summarizes these findings.

Table 4.3: RMIC Organization Integration, by CCO

\begin{tabular}{cccccccc}
\hline & \multicolumn{2}{c}{ CCO A } & \multicolumn{2}{c}{ CCO B } & \multicolumn{2}{c}{ CCO C } \\
\hline & PC & MH & PC & MH & PC & MH \\
\hline $\begin{array}{c}\text { Organization Integration } \\
\begin{array}{c}\text { Inter-organizational } \\
\text { governance }\end{array}\end{array}$ & $1 \pm$ & $1 \pm$ & $1 \pm$ & $1 \pm$ & $1+$ & $1+$ \\
\hline
\end{tabular}




\begin{tabular}{lllllll}
\hline $\begin{array}{l}\text { Inter-organizational } \\
\text { strategy }\end{array}$ & $2 \pm$ & $2-$ & $2 \pm$ & $2-$ & $2+$ & $2 \pm$ \\
\hline Trust & $2 \pm$ & $2 \pm$ & $2+$ & $2 \pm$ & $2+$ & $2+$ \\
\hline
\end{tabular}

$0=$ not mentioned, $1=$ alluded to, $2=$ directly discussed

$+=$ helpful, $-=$ not helpful, $\pm=$ mixed

Governance boards across the CCOs are similar in representation. This is largely because OHA policy required that $\mathrm{CCO}$ governing boards consist of the representatives from major components of the health care system, including at least one representative from primary care and one from behavioral health (HB $3650 ; 2011)$. Likewise how key informants discussed the importance of trust was also similar across the CCOs; within all three CCOs, building and maintaining trust among community partners was identified as critical. However, in many regards, the three CCOs are distinct from each other, differing along characteristics such as size, geographic service area, and degree of complexity. Each also has a unique structure and approach to engaging with its governance board, providers, and clinics, which in turn shapes decision-making as well as the CCO's strategy for facilitating mental health and primary care integration.

CCO A opted to retain much of the pre-existing structure of the health care system with the exception of integrating the counties' mental health systems into a single regional behavioral health network. While this was due to concerns about maintaining the stability of the health care system and was a response to the size and complexity of the service area, it has made it challenging to coordinate activities among all the partners. In addition, it creates a level of division between $\mathrm{CCO}$ A and clinical efforts to integrate primary care and mental 
health. Finally, it also assumes that independent organizations with separate business interests will work towards the greater good.

In contrast, $\mathrm{CCO} B$, which serves a much smaller geographic service area, chose to create a distinct structure in which the governance board and the CCO are separate entities. This choice was partially based on the recognition that building and maintaining trust in the small rural community was very important. Thus, the governance board is given considerable authority to make decisions about the direction of CCO B's strategy and many of the meetings are open in order to promote transparency. This has been very effective at building and maintaining trust and ensuring that $\mathrm{CCO} B$ is aligned with provider and clinic concerns regarding the integration of primary care and mental health. CCO B's high degree of awareness of provider and clinic concern is demonstrated by the CCO's focus on developing payment models that can support incremental transitions as many of its clinics are small, private clinics that find it difficult to change practice within a multi-payer system. While the relationship between $\mathrm{CCO} \mathrm{B}$ and its governance board results in a very responsive $\mathrm{CCO}$, this structure also gives a considerable amount of influence in strategy and decision-making to the very stakeholders who may need to be coaxed into initiating practice change.

As with $\mathrm{CCO} A$ and $\mathrm{CCO} B, \mathrm{CCO} C$ also stressed the importance of building and maintaining trust with its partners and other health care stakeholders in the community. In contrast to the other two CCOs, however, $\mathrm{CCO} \mathrm{C}$ is actually very engaged with providers and clinics and has a strong internal 
philosophy about the role of the $\mathrm{CCO}$ in supporting providers and clinics in integrating primary care and mental health. In particular, key informants from $\mathrm{CCO} \mathrm{C}$ reported that the $\mathrm{CCO}$ has a strong consultation model that places provider and clinic capacity as central to the ability to integrate primary care and mental health. Thus, CCO C places specialists into the clinics to help providers develop a better understanding of how to provide team-based care as well as determine what integration means to them. This approach emphasizes the ambiguous and vague nature of the term "integration" and gives providers and clinics guidance in operationalizing integration in very practical and applied ways that take into consideration the context of the clinic, providers, and larger community.

Professional Integration. In terms of integrating primary care services into mental health settings, $\mathrm{CCO} A$ and $\mathrm{CCO} B$ scored the same across education and training (2士), indicating that key informants from both CCOs directly discussed the feature but it was discussed as both a barrier and facilitator. $\mathrm{CCO} C$ scored a $2+$ for education and training indicating that key informants directly discussed the feature and that it was helpful; in fact, CCO C actively promotes education and training via its consultation model. Both CCO A and CCO C scored a 0 on agreements on interdisciplinary collaboration; this did not manifest in the data. CCO B alluded to a partnership between an FQHC and the community mental health program that was helpful in integrating mental health services in a local FQHC. Finally, all three CCOs scored a 2+ for value 
creation for the professional indicating key informants directly addressed value creation and that it was spoken of as a facilitator for integrating mental health services into primary care settings.

In terms of integrating primary care into mental health settings, both $\mathrm{CCO}$ A and CCO B scored the same across education and training (1-), agreements on interdisciplinary collaboration (0), and value creation for the professional (1-), indicating that education and training and value creation were alluded to but not directly addressed, that both are barriers, and that agreements on interdisciplinary collaboration were not mentioned. CCO C scored a $2 \pm$ for both education and training and value creation, and a 0 for agreements on interdisciplinary collaboration, indicating key informants directly addressed education and training and value creation but not agreements on interdisciplinary collaboration. These features were scored as mixed in regards to whether they were helpful or not; again, this is because while CCO C's workaround was helpful in one setting, it has not been spread to all mental health settings. Table 4.4 summarizes these findings.

Table 4.4: RMIC Professional Integration, by CCO

\begin{tabular}{lccccccc}
\hline & \multicolumn{3}{c}{ CCO A } & \multicolumn{2}{c}{ CCO B } & \multicolumn{2}{c}{ CCO C } \\
& PC & MH & PC & MH & PC & MH \\
\hline Professional Integration & & & & & & \\
\hline$\quad$ Education and training & $2 \pm$ & $1-$ & $2 \pm$ & $1-$ & $2+$ & $2 \pm$ \\
\hline $\begin{array}{l}\text { Agreements on } \\
\text { interdisciplinary collaboration }\end{array}$ & 0 & 0 & $1+$ & 0 & 0 & 0 \\
& $\begin{array}{l}\text { Value creation for the } \\
\text { professional }\end{array}$ & $2+$ & $1-$ & $2+$ & $1-$ & $2+$ & $2 \pm$ \\
\hline $\begin{array}{l}0=\text { not mentioned, } 1=\text { alluded to, } 2=\text { directly discussed } \\
+=\text { helpful, }-=\text { not helpful, } \pm=\text { mixed }\end{array}$ & & & & & & \\
\hline
\end{tabular}


At the professional level, education and value creation for the professional were similarly discussed by key informants from all three CCOs. These two elements were particularly related to how historically disparate professional cultures have made it challenging for primary care and mental health providers to work together as a team to deliver whole-person care. There was a sense among most of the key informants from all three CCOs that this was starting to change, albeit slowly. There were also considerable concerns about the competencies and skill sets necessary for mental health providers working in primary care. The feature, agreements on interdisciplinary collaboration, was not mentioned with the exception of CCO B which alluded to a formal agreement between the community mental health program and a local FQHC to place mental health providers from the community mental health program into the FQHC. Finally, both CCO A and CCO B, due to their structures and their relationships with either their partners (CCO A) or their governance board (CCO B), had limited engagement at this level. Of all three CCOs, CCO C was the most engaged at the professional level; this was largely the result of the strong consultation model it employed to work with providers and clinics.

Clinic level. While key features (i.e., case management, continuity, interaction between professional and client, and individual multidisciplinary care plans) were alluded to in interviews and in key documents for integration of mental health services into primary care settings, none of the CCOs directly discussed them and thus these features were scored as 1 across all three. CCO 
C was the only CCO that appeared strongly engaged at this level. Therefore, while CCO C's key informants did not necessarily go into great detail about these key features, they did discuss how important it was to work with providers and clinics to help them figure out how to provide team-based care and support them while they figured out and clarified roles and responsibilities for primary care and mental health providers.

In terms of integrating primary care services into mental health settings, the key features did not manifest in data for $\mathrm{CCO}$ A or CCO B. They were alluded to during interviews with key informants from CCO C but were scored as mixed ( \pm ); while there was a workaround in place for bringing primary care services to the community mental health program, this workaround was limited to just this one setting. Table 4.5 summarizes these findings.

Table 4.5: RMIC Clinical Integration, by CCO

\begin{tabular}{|c|c|c|c|c|c|c|}
\hline & \multicolumn{2}{|c|}{ CCO A } & \multicolumn{2}{|c|}{$\mathrm{CCOB}$} & \multicolumn{2}{|c|}{ CCO C } \\
\hline & PC & $\mathrm{MH}$ & PC & $\mathrm{MH}$ & PC & $\mathrm{MH}$ \\
\hline \multicolumn{7}{|l|}{ Clinical Integration } \\
\hline Case management & $1-$ & 0 & $1-$ & 0 & $1+$ & $1 \pm$ \\
\hline Continuity & $1-$ & 0 & $1-$ & 0 & $1+$ & $1 \pm$ \\
\hline $\begin{array}{l}\text { Interaction between } \\
\text { professional \& client }\end{array}$ & $1-$ & 0 & $1-$ & 0 & $1+$ & $1 \pm$ \\
\hline $\begin{array}{l}\text { Individual } \\
\text { multidisciplinary care } \\
\text { plan }\end{array}$ & $1-$ & 0 & $1-$ & 0 & $1+$ & $1 \pm$ \\
\hline
\end{tabular}

What was not necessarily captured by the RMIC framework, one finding that emerged in the data was the importance of the $\mathrm{PCPCH}$ model for facilitating integration of mental health services in primary care. At the clinic level, its 
importance was primarily in providing clinics with a roadmap they could follow to get started. It is worth noting that many of the key features of the clinic level of integration are aligned with the standards of the PCPCH model. Thus clinics striving to implement the PCPCH model do take into consideration the importance of case management, continuity of care, relationships between providers and patients, and individual multidisciplinary care plans.

Integration of primary care services in mental health settings was extremely limited. In CCO A and CCO B, discussions centered on the desire for some sort of standards to define what integrated care in mental health clinics looks like; key informants from CCO B explicitly mentioned that they were monitoring the $\mathrm{CCBHC}$ demonstration program as they wanted to align future efforts to integrate primary care in mental health settings with this program. CCO C demonstrated the most progress in integrating care in mental health settings. It achieved this by partnering with local health care stakeholders to co-locate an advanced $\mathrm{PCPCH}$ in the same building as the community mental health program to ensure delivery of primary care services for individuals with SMI.

\section{Enablers of Integration}

The presence or absence of enablers of integration can not only impede or facilitate the integration of health care services but also serve as binding factors to bring together multiple levels of integration. According to the RMIC framework, there are two categories of enablers (normative and functional). Shared vision, reliable behavior, linking cultures, and visionary leadership are 
key normative enablers; learning organizations, information management, and regular feedback of performance indicators are key functional enablers.

Normative enablers are primarily about developing relationships built on trust and generating buy-in and commitment. It is difficult to talk about the key features as distinct because of the high degree of inter-relatedness. However, shared vision and visionary leadership are really about the ability to generate commitment and buy-in for an ideal future state. Linking cultures and reliable behavior emphasize relationship building, a necessary component for not only generating but also maintaining commitment. Together these enablers are essential for the development of a shared understanding of what integration of primary care and mental health is in practice.

At the system level, normative enablers were present and each of the CCOs was actively engaged in the development of normative enablers at the organization level for integrating mental health services into primary care settings. However, CCO A, due to limited engagement with providers and clinics, did not drive the development of normative enablers at the professional and clinic levels. CCO B attempted to do so through its governance board and emphasis on community driven priorities and decision-making. However, this also resulted in very slow change as many of their small, private clinics indicated reluctance to change practice. In contrast, CCO C successfully developed normative enablers at the professional and clinic levels in ways that supported and facilitated the integration of primary care and mental health. CCO C's consultation model was 
a response to an obvious lack of understanding of what primary care and mental health integration actually means in practice. By placing specialists directly in clinics to work with providers, CCO C not only acknowledges that changing practice is hard but actively maintains and supports relationship building between the $\mathrm{CCO}$ and clinics, between primary care and mental health providers, and between providers and patients. This also helps the clinics to envision a future ideal state of primary care and mental health integration.

Functional enablers, on the other hand, are important for the actual implementation of integration. These enablers explain what tools and resources are needed in order to implement and support integration. Again, the interrelatedness between features makes it difficult to disentangle them from each other. Information management and regular feedback of performance indicators describe the mechanisms in place to collect and communicate important information and data. Valentijn's (2015) concept of learning organizations is more narrowly defined than Senge's concept of learning organizations (1990) and focuses on information collection and feedback loops. Valentin's learning organization encompasses the notion that stakeholders throughout the system should collect information to help inform integration efforts as well as listen to feedback and respond in ways that support overall goals for integrating mental health and primary care (2015).

For all the CCOs studied, there were moderate to high levels of functional enablers present at the system level and each of them were engaged in 
developing those enablers at the organization level. Since CCO A does not engage with providers and clinics and instead delegates that work to its partners, it does not drive the development of functional enablers down into the professional and clinic levels. CCO B focused on building trust and thus created mechanisms to receive feedback; however, in terms of thinking through how to support providers and clinics in integrating primary care and mental health, the CCO has worked almost exclusively on how to create transitional payment models to promote integration. Without an understanding of what integration means, it is difficult to develop functional enablers that meaningfully promote and support integration of primary care and mental health. In contrast, $\mathrm{CCO} \mathrm{C}$ is highly engaged in developing functional enablers at the professional and clinic levels with its consultation program that not only works with clinics and providers to help them figure out what integration means, but also works closely with them to actually test various ways to implement different strategies in order to discover what works best for clinics, providers, and patients.

\section{Scope of Integration}

Scope of integration refers the extent that the integration of primary care and mental health is "either person-oriented or population-oriented" (Valentijn et al., 2015). Valentijn and his colleagues suggest that while the two may seem at odds with each other, strength of integration effort is grounded in a balance between the two (2015). Initially the researcher thought that there might be some variation in the scope of integration for each CCO. However, the three 
CCOs included in this study tended to be more population-oriented. CCOs are largely designed to focus on population health. The $\mathrm{PCPCH}$ model brings a person-oriented approach to health reform in Oregon, but in an indirect manner (Rissi et al., 2014).

\section{Additional Themes}

There were a number of themes that emerged from the data and are embedded throughout the findings that were not adequately predicted nor necessarily captured by the RMIC framework, but were nevertheless important. This section highlights these so they do not get lost in the narrative. They will be discussed in greater detail in the discussion chapter.

First, while the RMIC enabled the researcher to identify the important role that the $\mathrm{PCPCH}$ model played in facilitating integration in primary care settings, the model does not fit neatly into the RMIC. It was also a factor that did not fit neatly into any one level nor did it fit well as either a functional or normative enabler. Second, the expectation that CCOs facilitate integration of care for individuals with SMI obscures the fact that the mental health system is not as equally developed as the primary care system. Third, there has yet to be a system level discussion in Oregon about where individuals are best served (i.e., primary care or mental health). Finally, there is a great need for meaningful measures of integration, particularly ones that are relevant for mental health settings. 


\section{Conclusion}

The study was intentionally designed to focus on the CCO perspective. Each of the CCOs was unique in terms of how they interacted with their partners and provider network. This resulted in variation in the degree of detail collected about each level of integration, as there was variation in the degree of $\mathrm{CCO}$ involvement. In addition, this study addressed understanding to what extent CCOs were involved in facilitating integration in both primary care and community mental health settings; however, interviews revealed that integration in community mental health settings is extremely limited and, at least in regards to these three cases, not necessarily driven by the CCOs. Thus, much of the findings focus on integration of mental health services in primary care settings.

The theory of Complex Adaptive Systems (CAS) posits that boundaries in a CAS are fuzzy (Gioia \& Chittipeddi, 1991; Plsek \& Greenhalgh, 2001); thus activities and factors do not always neatly fall into only one level. It was challenging at times to determine the level into which emerging themes should be sorted. Ultimately, themes were organized into levels according to perceived impact, and not necessarily origin or domain of activity. This way of framing the results also helped address a conundrum presented by studying only the organizational perspective: the reality that almost everything that emerged during interviews could be sorted into one level -- in this case, the organization level of integration. There are many ways to frame information; this is simply one. 
Applying the RMIC to real-world efforts provided a rich opportunity to test the usefulness of the framework for understanding and assessing integration efforts. For the most part, the RMIC framework and taxonomy provided a useful tool for analysis of CCO stakeholder perspectives; however, there were some limitations that will be discussed in the next chapter. In addition, Chapter 5 will also present a number of recommendations for future efforts to facilitate and support the integration of primary care and mental health. Integration of care is increasingly touted as one of the most promising approaches to achieve the Triple Aim. Thus, refining frameworks to assess, strategize, and monitor efforts to integrate care is essential and timely. 


\section{Chapter 5: Discussion}

The purpose of this study was twofold: (1) to explore the factors that impact the ability of CCOs to facilitate the integration of primary care and mental health and (2) to assess the utility of the RMIC framework for exploring integration of mental health and primary care in real world settings.

The RMIC seeks to provide a representation of the different components necessary to support the integration of primary care and specialty health services while acknowledging the complexity of the health care system. It draws attention to the interconnectedness among all the levels of the health care system and implies that clinical integration is more likely to be successful when key factors within the system, organizational, professional, and clinical levels are aligned. Normative and functional enablers are critical for developing and maintaining this alignment both within and across levels. The RMIC highlights that these enablers as well as the processes and factors found at every level need to be carefully assessed, managed, and monitored.

This chapter starts with a summary of the findings that articulate the current state of integration within Oregon CCOs studied based on the application of the RMIC model. Early analysis revealed that direction matters (i.e., mental health into primary care settings and primary care into mental health settings). Thus, there is an emphasis on explaining the implications of findings related to the direction of integration efforts. This section also addresses a number of themes that emerged from the data but were not reflected in the RMIC. Then the 
research question is revisited with a discussion of how the study responded to this question. This is followed by a discussion of the RMIC's perceived usefulness as a framework for exploring and assessing integration efforts.

Finally, the chapter concludes with implications for policy, limitations of the study, and future research recommendations.

\section{Summary of Findings}

The key finding from this study is that there was variation in level of integration processes across the CCOs. In addition, the RMIC was successful in identifying those variations as well as in differentiating extent of $\mathrm{CCO}$ integration of primary care and mental health; CCO C was the most advanced in terms of integrating primary care and mental health, followed by $\mathrm{CCO}$ B and then CCO A. In addition, this variation was due to differences in the level of engagement in developing normative and functional enablers among the CCOs.

All three CCOs had moderate-high levels of normative and functional enablers at the system level. However, because of the structure of CCO A and the relationship between the $\mathrm{CCO}$ and its risk-bearing partners, it had limited interaction with stakeholders at the professional and clinical levels and thus did not actively drive the development of normative and functional enablers down to those levels. CCO B was aligned with provider and clinic needs and concerns and highly emphasized the importance of building trust as evidenced by open meetings and listening sessions; however, $\mathrm{CCO}$ B struggled with developing and implementing a strategic plan that operationalized and supported the integration 
of primary care and mental health. Its efforts have centered mostly on developing transitional payment models to support practice change in a multi-payer system, but the clinics themselves seem to remain resistant. $\mathrm{CCO} C$ is the only $\mathrm{CCO}$ that has explicitly driven the development of both functional and normative enablers down to the professional and clinic levels. The CCO's consultation model deals explicitly with helping clinics work out what integration of primary care and mental health looks like and what it is that clinics and providers need to implement it. Table 5.1 provides a summary of these findings.

Table 5.1: RMIC Enablers, by CCO

\begin{tabular}{ccccccc}
\hline & \multicolumn{2}{c}{ CCO A } & \multicolumn{2}{c}{ CCO B } & \multicolumn{2}{c}{ CCO C } \\
\hline Integration Level & $\mathrm{N}$ & $\mathrm{F}$ & $\mathrm{N}$ & $\mathrm{F}$ & $\mathrm{N}$ & $\mathrm{F}$ \\
\hline System & + & + & + & + & + & + \\
\hline Organization & + & + & + & + & + & + \\
\hline Professional & - & - & + & - & + & + \\
\hline Clinical & - & - & + & - & + & + \\
\hline
\end{tabular}

$+=$ present, $-=$ not present

$\mathrm{N}=$ Normative enablers, $\mathrm{F}=$ Functional enablers

CCO C's consultation model is particularly notable because while key informants from each $\mathrm{CCO}$ indicated that many of their clinics have adopted the PCPCH model, adoption of the PCPCH model does not necessarily equate to integration. Interview data revealed that while key informants reported that many primary care clinics have hired and embedded behaviorists in their operations, there were lingering concerns such as workforce competency (i.e., do providers know how to work in teams and are their roles clearly defined?) and clinic capacity (i.e., are payment models financially sustainable in a multi-payer system and do clinics have the capacity to meet the extra administrative burden?). By 
embedding consultants in the clinics, $\mathrm{CCO} \mathrm{C}$ directly works with the clinics to help them work through both of these concerns. The process of supporting the development of normative and functional enablers throughout all levels of the system is important for creating alignment as stakeholders determine what integration of primary care and health actually is, as well as identifying what it is that providers and clinics need to do to actually integrate care.

The important role of structural models. The findings also suggest that structural models such as the PCPCH model can play a critical, though not always sufficient, role in facilitating the integration of primary care and mental health. The RMIC indicates that alignment across all levels of the health care system is likely to facilitate integration initiatives and that normative and functional enablers are necessary to develop that alignment. However, findings from this study indicate that it is also helpful if a mechanism exists that facilitates the development of these enablers across the different levels of the health care system. The PCPCH model served as a reference point for stakeholders across all levels of the system as they grappled with the concept of mental health and primary care integration.

Important to this process was the high alignment at the system level that anchored the PCPCH model throughout all levels of the system. This was evidenced by the state which incentivized CCOs to facilitate PCPCH adoption, by CCOs which used a variety of strategies (e.g., outreach, administrative support, technical assistance, incentive payments) to encourage clinics to adopt the 
model, and by clinics and providers that used the PCPCH standards to think about how to approach care redesign to support mental health and primary care integration.

The PCPCH model served as the mechanism that enabled normative and functional enablers to develop and evolve. As stakeholders across the levels of the health care system attempted to facilitate or implement the PCPCH model, they engaged in conversations about what primary care and mental health integration means and what it looks like in practice, at the same time as they discussed what was needed to implement and support integration. These conversations in turn continually influenced each other, shaping the development and implementation of strategies to integrate primary care and mental health.

Figure 5.1 provides a visual representation of this process and the relationship between levels and normative and functional enablers of integration. The figure is based on Valentijn's RMIC (2013) and the findings from this study; it demonstrates the binding characteristics of the PCPCH model as well as the feedback loops that facilitate the development of normative and functional enablers of integration within and across the levels of the health care system. The double-sided arrow that connects normative and functional enablers of integration emphasizes the dependent relationship between the two sets of enablers of integration and the necessity of having both present and actively managed to facilitate and support integration of primary care and mental health. 
In CCO C, which was the only CCO to drive both normative and functional enablers down to the professional and clinic levels, the $\mathrm{PCPCH}$ was a particularly powerful tool for facilitating integration of primary care and mental health. The PCPCH model served as a foundation for $\mathrm{CCO}$, its partners, providers, clinics, and other health care stakeholders to both develop a sense of what integration actually means in practice for both providers and patients as well as identify what clinics and providers need to provide integrated care.

Figure 5.1: RMIC and the Role of Structural Models

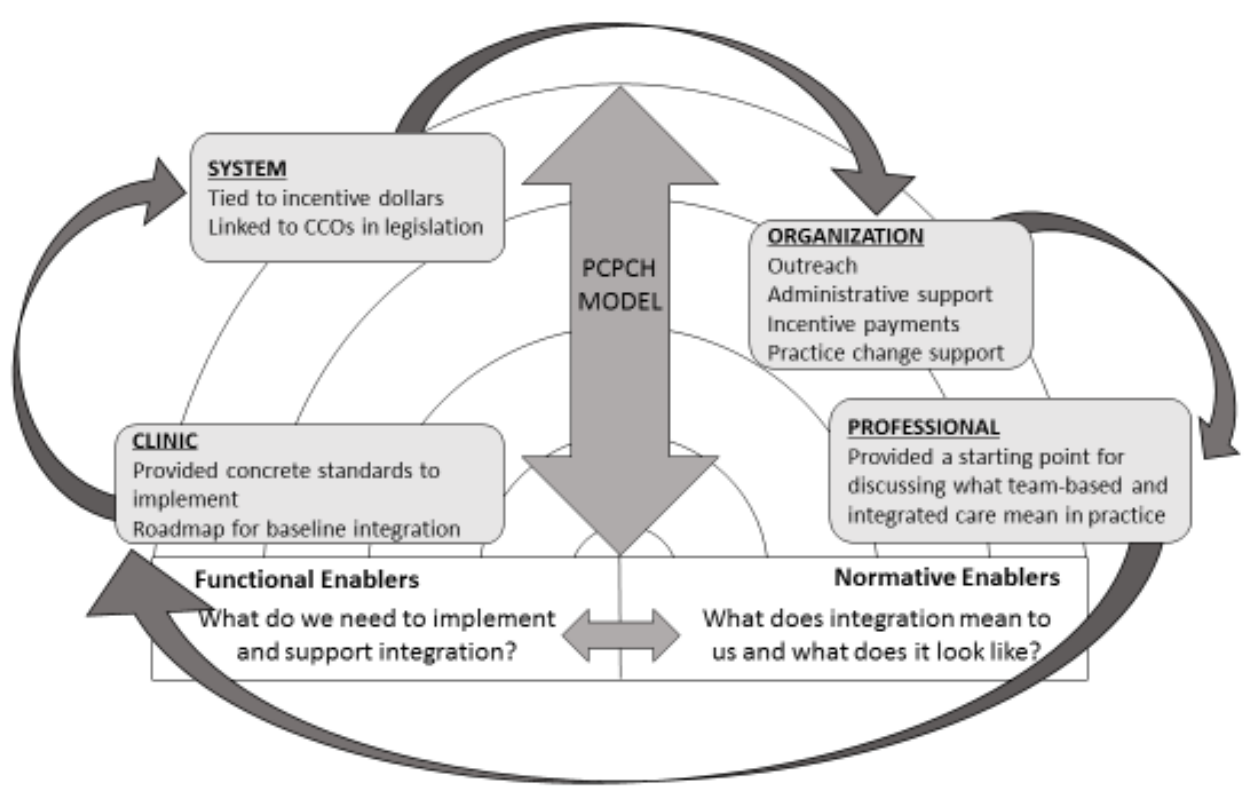

Integration of primary care services into mental health settings is

limited. Integration of primary care into mental health settings was extremely limited despite wider health transformation narratives that stressed the importance of integrating care for individuals with SMI. Two of the CCOs indicated that they had yet to try to facilitate widespread integration of care in 
mental health settings. CCO $\mathrm{C}$ was the most integrated, by partnering with other community stakeholders to set up an advanced PCPCH housed in the same building as the county mental health program to deliver primary care services to individuals with SMI and substance use disorders. This form of partnership more closely matches what is referred to as "co-located care" from the SAMHSAHRSA conceptual framework of collaboration and integration (Gerrity, 2016) (see Table 2.2 in Chapter 2).

The absence of concerted efforts to integrate primary care in mental health settings reinforced the importance of a structural model such as the $\mathrm{PCPCH}$ model for facilitating integration of mental health services in primary care settings. Currently there are no well-developed companion models to identify what integration means nor to guide efforts to integrate primary care into mental health settings.

However, it is important to note that the CCBHC demonstration program is intended to facilitate the development of a payment and structural model to guide the integration of primary care services into behavioral health clinics. Clinics from two of the $\mathrm{CCOs} \mathrm{(CCO} \mathrm{A} \mathrm{and} \mathrm{CCO} B$ ) are actively participating in the demonstration program. While $\mathrm{CCO} \mathrm{C}$ does not have any clinics that are participating, key informants from $\mathrm{CCO} C$ reported the $\mathrm{CCO}$ is carefully watching the development and implementation of the $\mathrm{CCBHC}$ demonstration program to align future efforts with the model and standards that will be developed. 
CCO C's experience establishing co-located care for individuals with SMI further suggests that structural models are critical for facilitating integration of mental health and primary care. It came the closest, as compared to the other two CCOs, to achieving integration in mental health settings by utilizing the PCPCH model to bring primary care services to individuals with SMI and substance use disorders.

In addition, key informants identified a number of factors that limited CCO ability to facilitate the integration of care into mental health settings, including misaligned regulatory frameworks, lack of financial incentives, disparate professional cultures, and workforce and clinic capacity. These factors are embedded throughout the system, organization, professional, and clinic levels. However, these issues point to a limitation of the RMIC framework: the fact that the mental health system and primary care system are not equally developed. Struggling with issues of access and quality, the mental health system is simply not as stable as the primary care system. Thus it is not entirely surprising that key informants from all three CCOs discussed efforts to increase access and improve quality.

Important themes not captured by the RMIC framework. Finally two other themes emerged from the data but were not accounted for by the RMIC framework; these are: 1) the need for a broader discussion about where individuals are best served (i.e., primary care or mental health) and 2) the need 
for meaningful metrics to measure integration of primary care and mental health that account for different settings.

Integration of primary care and mental health at its simplest is the idea that both the mental health as well as the primary care needs of individuals are recognized, respected, and met by the health care system. In practice, this involves primary care clinics with integrated mental health services that provide screening, identification and intervention, and care management for mental health concerns. In addition, it requires that community mental health settings have integrated primary care services to provide screening, identification and intervention, and care management for chronic physical conditions. Finally, primary care settings and community mental health settings should have mechanisms in place for communication about shared clients and referrals for complex cases that are beyond the scope of the referring clinic.

In order to achieve this vision of a health care system with integrated primary care and mental health, it is necessary to develop general guidelines for how people should ideally move through the system. However, interview data revealed that, at the system level, a conversation about where people are best served has yet to occur.

This lack of a larger discussion has implications for the ability of CCOs to effectively assess, manage, and monitor the integration of primary care and mental health, as well as implications for the ability of clinics to effectively collaborate to ensure individuals have their needs met, no matter where they 
present with concerns. Just as in a single clinic setting where clarification on roles and responsibilities is essential for team-based care, it is essential that both primary care and community mental health have a shared understanding of their roles and responsibilities in making referral decisions. This shared understanding will require clarification regarding when individuals' health concerns (both physical and mental) are best managed in one setting over the other.

Finally, the lack of meaningful metrics for integration was raised multiple times during interviews. However, there is not a clear definition of what integration is, what it means, and what it looks like, let alone what metrics should be used to measure it. Instead, organizations, clinics, and researchers frequently rely on process or proxy measures that may suggest integration is occurring. For example, $\mathrm{PCPCH}$ recognition was included as an incentive metric by $\mathrm{OHA}$ and in many ways serves as a proxy measure for integration of mental health services in primary care settings. However, as previously noted, PCPCH adoption does not necessarily equate to integration of mental health and primary care and thus its utility as a proxy measure of integration is limited.

\section{The Research Question}

The key research question for the study was: What key factors in Oregon's health care system impede or facilitate the ability of Coordinated Care Organizations to encourage the integration of primary care and mental health? This study found that the ability of organizations to develop normative and 
functional enablers and ensure that integration processes are aligned across the levels of the health care system is a critical factor for integrating primary care and mental health. Contrary to the literature review in Chapter 2 which pointed to a specific list of factors related to organizational structure, organizational and professional culture, technology, payment and reimbursement, and regulatory frameworks; the key factors illuminated in this study were more complex. While the study confirmed that factors related to organizational structure, organizational and professional culture, technology, payment and reimbursement, and regulatory frameworks are important and do have an impact on how integration initiatives are implemented, this study also found that what is perhaps most important is the ability of stakeholders throughout the system to both articulate what integration means as well as identify what is needed to support it.

\section{RMIC Utility}

The RMIC framework provided a useful framework for exploring integration efforts, was particularly helpful in distinguishing different levels of integration processes among the three CCOs included in this study, and enabled the researcher to identify that $\mathrm{CCO} C$ was the furthest along in terms of actively initiating and supporting integration of primary care and mental health. In particular, its focus on the multiple levels of the health care system highlights interdependence among the levels. Thus, the framework supports a comprehensive view of the integration of primary care and mental health and emphasizes the importance of alignment across and within levels of the health 
care system. In addition, it offers two concepts to the larger body of literature on primary care and mental health integration: (1) normative enablers of integration and (2) functional enablers of integration. Finally, because it takes into consideration the impact of factors embedded within and across multiple levels of the health care system, it promotes a thorough assessment that may prove useful for forecasting and identifying potential opportunities and challenges.

However, the RMIC framework had some limitations. While differentiating among the levels of the health care system at which integration processes occurs was helpful for "telling the story" of integration for each CCO as well as identifying both successes and challenges, the taxonomy for the normative and functional enablers of integration proved challenging to apply. It was clear from the data that normative and functional enablers of integration were important for facilitating integration processes, but the features used to characterize the enablers are so inter-related that it made it challenging to isolate and characterize meaningfully as separate features.

In addition, while it made intuitive sense that features such as trust and reliable behavior are essential for successful collaboration among partners, the researcher struggled to make sense of them in a way that was meaningful and helpful. Ultimately, the researcher found that a better understanding of normative and functional enablers of integration situates the concepts within a practical framework that emphasizes the question, "to what purpose?" 
Thus, normative enablers of integration which include features such as trust, reliable behavior, shared vision, and visionary leadership are contextualized as factors that are necessary in order for stakeholders to come to some shared understanding about the meaning of integration of primary care and mental health. Similarly, functional enablers of integration which include features such as learning organization, information management, and regular feedback of performance indicators are contextualized as factors that are necessary in order for stakeholders to understand what it is they need to implement integration and assess if they are achieving their goals.

Once the researcher was able to step back from her initial assumptions about these concepts, she was able to approach them less as a "box to be checked" and more as a collection of features that have a collective impact for a particular purpose. This enabled her to assess the data and identify how these factors work to bring together different levels of the health care system in a manner that facilitates and supports the integration of primary care and mental health. Once this issue was resolved, the RMIC provided a useful framework to assess $\mathrm{CCO}$ degree of involvement in developing functional and normative enablers of integration at different levels of the health care system.

\section{Implications for Policy}

CCOs are organizations that are responsible for improving the financing, organization, and delivery of health services for the population they serve. Thus, they are not only well positioned but specifically tasked with the responsibility of 
driving conversations about what integration of primary care and mental health means in the context of their communities as well as identifying what clinics and providers actually need to implement it.

These are not easy or simple tasks and require that CCOs balance the tension of managing evidence with innovation, accountability with flexibility, topdown directives with buy-in from below, and individual stakeholder goals with overall CCO goals. Such tensions or paradoxes are to be expected in CAS (Plsek \& Greenhalgh, 2001; Begun, Zimmerman, \& Dooley, 2003; Anderson \& McDaniel, 2000; Smith \& Lewis, 2012). The literature on CAS suggests that these tensions require that leadership be transparent about what is not known, focus on building relationships, and continually engage in collective sensemaking (Anderson \& McDaniel, 2000; Weick \& Sutcliffe, 2007; Smith \& Lewis, 2012; Maitlis \& Christianson, 2014). The latter refers to the process through which individuals work through ambiguity to create meaning; this process has been connected to other important organizational processes including strategic change, learning, and innovation (Maitlis \& Christiason, 2014).

CCOs are expected to facilitate integration of primary care and mental health in the absence of a well-articulated definition of integration. There is both pressure to utilize evidence-based models and practices and yet an expectation to be flexible to account for unique community context and needs. In a similar fashion, clinics are expected to promote the use of team-based care, but this study's findings indicate that there is some confusion regarding what team-based 
care actually means and looks like in practice. This lack of a common understanding of "integration of primary care and mental health" requires that CCOs engage their partners, clinics, and providers in an open and transparent conversation about what is known and what is not known in order to facilitate a process through which they can collectively build a shared understanding of what integration means as well as identify desired outcomes and preferred strategies.

The RMIC's normative and functional enablers of integration offers a new approach to navigating these tensions, one that recognizes and allows for the simultaneous creation of what integration of primary care and mental health care means in practice as well as a broader understanding of what tools, resources, and information are needed to actually do the work of integration. This study's findings suggest that $\mathrm{CCO}$ can play an important role in developing normative and functional enablers of integration to bring stakeholders at multiple levels of the health care system together to engage in collective sensemaking.

Findings from this study highlight the importance of the convergence of normative and functional enablers of integration to facilitate integration of mental health and primary care. The PCPCH program emerged as a key mechanism for facilitating this convergence. Integrated with health transformation and CCOs in Oregon legislation and included as a $\mathrm{CCO}$ incentive metric, the $\mathrm{PCPCH}$ recognition process gave CCOs a starting point from which to tackle the issue of integration in primary care settings, and provided clinics with a roadmap for thinking about how to redesign care to support integration. In contrast, efforts to 
facilitate integration of primary care into mental health settings were found to be extremely limited. The findings from this study suggest that the instability of the mental health system and the lack of a structural model to guide integration efforts in mental health settings are significant barriers. The need for a behavioral health model, similar to the $\mathrm{PCPCH}$ model, is evident.

Current federal efforts via the $\mathrm{CCBHC}$ demonstration program offer a promising opportunity to develop and test standards and payment models to support the integration of primary care services in behavioral health settings. Oregon should continue to participate in and monitor these efforts. If the CCBHC demonstration program successfully develops a model for integration in behavioral health settings, OHA should support implementation and incentivize CCOs to increase adoption of the model in a manner similar to what it did for PCPCHs.

The findings from this study also demonstrate that there are a host of concerns related to community mental health programs and mental health providers in both mental health and primary care settings. These concerns include instability of the mental health system, disparities in wages, administrative burden, and uncertainty on how administrative rules apply to mental health workers in primary care settings. Many of these concerns cannot be adequately addressed by $\mathrm{CCO}$ acting in isolation and instead require the state to provide leadership and guidance. 
In 2016, OHA convened the Behavioral Health Collaborative for the purposes of developing a list of recommendations for improving and strengthening the behavioral health system (OHA, 2016). Recommendation 3 pertains to the workforce and provides an opportunity for $\mathrm{OHA}$ to engage with the broader behavioral health system and workforce to lead a discussion about how these issues impact access and quality as well as to prioritize these concerns and determine the best approach to address them. Ultimately, it will be fundamentally impossible to achieve the full goals of primary care and mental health integration unless these issues of system instability are addressed.

Given the findings from this study that there is variation in the degree to which CCOs are engaged with facilitating and guiding integration efforts, $\mathrm{OHA}$ could potentially consider metrics to measure, track, and assess the degree of active engagement among CCOs. However, these measures would need to be carefully designed in order to reduce the likelihood of unintended consequences. While this study's findings suggest that incentive metrics can be an effective tool, the findings also indicate that incentive metrics can potentially promote "teaching to the test" mentalities as well as unintentionally disadvantage and penalize some organizations over others.

Finally, the findings from this study highlighted the need for a potentially state-wide, but at the very least regional, conversation about the best setting of care for individuals with different risk and complexity of physical and mental health concerns. The lack of a shared understanding about the best setting of 
care is likely connected to the historical context of system fragmentation and lack of communication. However, it is essential that OHA, CCOs, and mental health authorities engage appropriate stakeholders including both primary care and mental health providers and the community in creating guidelines that while not necessarily prescriptive could provide guidance on ideal pathways for individuals based on their physical and mental health needs and preferences. The 4 Quadrant Clinical Integration Model and the SAMHSA-HRSA Framework of Collaboration and Integration (see Figure 2.1 and Table 2.2 in Chapter 2) could provide starting points for these discussions.

\section{Limitations of the Research}

There were a number of limitations of this study. First, the scope of the study was quite broad, which seriously limited the ability of the researcher to go into great depth about any one particular domain of interest. The research aims included developing a more comprehensive understanding of relationships between contextual conditions embedded within and across the system, identifying the factors that impact $\mathrm{CCO}$ ability to facilitate integration of primary care and mental health, and assessing the usefulness of the RMIC as a conceptual framework for guiding research and assessing integration processes. The broad scope resulted in a large amount of data and made it difficult to organize and report findings; however, the scope was appropriate for the aims of the study. 
The study was also simultaneously narrow in that it only looked at the integration of primary care and mental health services. CCOs are tasked with integrating primary, dental, and behavioral health care more broadly. Behavioral health care in Oregon is defined as services for both mental health and substance use disorders. The choice to not look at the integration of dental health care was a fairly early decision based on the researcher's interests in behavioral health care; however, the choice to carve mental health out of behavioral health was a more difficult decision.

While there is increasing evidence that substance use disorders have a significant impact on overall health (Whiteford et al., 2013; NIDA, 2017) as well as a growing awareness of the prevalence of comorbid mental illness and problematic substance use (Swendsen et al., 2010; Fenton et al., 2012; Wu \& Blazer, 2014; Grant, Saha, \& Ruan, 2016), including substance use disorder services would have introduced further complexity. While services for substance use disorders are siloed from primary care, they are also siloed from mental health care. It would be incredibly time consuming to disentangle how these multiple levels of fragmentation impact integration efforts. Focusing on efforts to integrate primary care and mental health and excluding services for substance use disorders for this study was primarily about feasibility, both in terms of time and available resources.

Second, the number of CCOs included in this multiple case study limits the external validity or the generalizability of the results. There are currently 16 
CCOs in Oregon; this study addressed only three. Each of those three were unique in terms of community demographics, organizational structure, and history of collaboration among health care leadership. There is a good chance that the remaining thirteen CCOs are equally as unique. Therefore it is possible that the themes that emerged from the study of the three CCOs included in this study may not be applicable to the other CCOs or similar organizations.

In addition, because the study limited interviews only to CCO key informants, the findings represent only perspectives from one group of stakeholders. Oregon's health transformation efforts involve a great number of stakeholders including but not limited to policymakers, providers, and the community. While every $\mathrm{CCO}$ is tasked with facilitating the integration of primary care and mental health, there are multiple parties involved in efforts to support and expand integration. It is important to stress that this is not the complete story of integration of primary care and mental health, which would require multiple perspectives. In addition, the limited number of interviewees from each CCO potentially provides only a partial picture of each CCO's efforts to facilitate integration. While a considerable number of CCO key documents and secondary interviews were included, the limited number of cases, the singular perspective, and the small number of participants all limit the generalizability of the findings.

Third, some may consider the qualitative design of this study to be less rigorous than that of a quantitative study. In particular there may be some concerns about validity and reliability. This concern is compounded by the fact 
that there was only one researcher to code and interpret the data. Due to limited resources and money, the researcher was unable to hire a second analyst to check code. Without a "check-coder" to test the reliability of the findings, there is an increased risk for confirmation bias, or only seeing what one expects to find and ignoring data that do not fit into the chosen conceptual framework. However, there are also various approaches a lone researcher can take to mitigate these concerns.

First, the researcher took field notes following interviews to highlight potential themes and interesting findings, and also kept detailed accounts of the coding process and analysis. This sort of reflexive practice requires the researcher to be perceptive to disconfirming evidence (Miles \& Huberman, 1994) and have an awareness of not just their thought processes but also the factors that influence their thinking (Darawsheh, 2014). The researcher employed a common technique referred to as triangulation or the use of multiple forms of evidence (i.e., primary interview data, secondary interview data, and key CCO documents); this is thought to increase validity as the method does not rely on a single form of evidence (Creswell \& Miller, 2000). In addition, the researcher utilized a modified version of "member checking" (Creswell \& Miller, 2000 Member checking is when the researcher shares key findings or emergent themes with the research participants in order to collect feedback on the credibility of the findings (Creswell \& Miller, 2000). However, this can also be highly inappropriate and lead to unintended consequences, particularly when 
participants are concerned about, and want to control, how they or the organization or group they represent are presented in the data (Mays \& Pope, 2000). Rather than conduct member checks with the participants, the researcher conducted multiple informational (off the record) interviews with stakeholders knowledgeable about $\mathrm{CCO}$ and integration of primary care and mental health to test emergent themes.

While these approaches do not eliminate all threats to validity and reliability, they do reduce the risk of confirmation bias. Finally, despite the limitations of qualitative research methods, the questions this study sought to answer cannot be answered through quantitative means. This study addressed understanding the contextual conditions that impact the ability of CCOs to facilitate integration of primary care and mental health. Quantitative methods are not particularly well suited to collecting information about contextual conditions (Yin, 2013). In addition, exploring differences in processes and perspectives in order to illuminate meanings related to these differences can fundamentally only be accomplished through qualitative methods (Yin, 2013).

\section{Recommendations for Future Research}

The findings from this study suggest several potential areas of research. Because of the broad scope of the study with its focus on the four levels of the health care system, many health care stakeholders, including policymakers, CCOs and similar organizations, local mental health authorities, primary care as well as community mental health programs, providers, and the community, would 
benefit from research that examines the gaps in knowledge and other issues identified by this study more closely. Potential future questions include:

1. How do the RMIC process findings from this study relate to outcome measures related to the integration of primary care and mental health? This study demonstrated clear variation in integration processes at different levels of the health care system. In order to fully understand the relationship between processes and outcomes, it would be beneficial to assess how findings from this study relate to outcome measures.

2. What are potential measures of integration of primary care and mental health that can be used to assess integration efforts in both primary care and mental health settings?

In order to achieve the previous research question, there is a great need for measures of integration that can be used to assess integration efforts in both primary care and mental health settings. Follow-up research, combining qualitative and quantitative methods, could operationalize and develop measures for primary care and mental health integration in order to explore whether or not CCOs that are actively engaged in developing normative and functional enablers at the organizational, professional, and clinic levels are able to facilitate greater integration of primary care and mental health.

3. What are the most promising strategies for embedding integration throughout the levels of the health care system? 
Findings from this study highlighted the importance of creating alignment among the different levels of the health care system to implement and support integration of primary care and mental health. Implementation research is needed to understand how to embed integration throughout the levels of the health care system.

4. What additional lessons can be learned from applying the RMIC to other areas of health care?

Findings from this study likely apply to other areas of health care as well as other organizational studies; it would be interesting to test whether they do apply and to determine what else can be learned from studying these concepts in other settings. For example, mental illness and substance use disorders are often lumped together under the term behavioral health; however, the system of care for each is distinct. A better understanding about the unique as well as common challenges in integrating each with primary care could help policymakers and other health care stakeholders develop strategies that are mindful of potential differences that require a more focused approach.

5. How do patients perceive and experience integration of primary care and mental health?

Noticeably missing from this study's findings are the experiences and perceptions of patients. This information could be used to help inform efforts to integrate primary care and mental health, incorporating patient priorities and recommendations to ensure that integration efforts are patient-centered. 


\section{Conclusion}

This study's goal was to better understand the key factors that impede or facilitate the integration of primary care and mental health. Using the RMIC, the researcher was able to assess CCO processes of integration and identify the variation among CCOs as well as which $\mathrm{CCO}$ was most advanced in terms of facilitating integration of primary care and mental health. Based on the initial literature review, the researcher expected to find that technology, payment and reimbursement models, and professional cultures were the main barriers to facilitating the integration of primary care and mental health.

While these factors were raised during the interviews, an unexpected finding that emerged from the data was the important role structural models such as the $\mathrm{PCPCH}$ model can play in facilitating the development of functional and normative enablers. Structural models can serve as a mechanism that enables stakeholders across the health care system to engage in discussions that define and clarify what integration means as well as identifies what is needed to facilitate and support it.

These conversations in turn can help to create alignment and a shared vision for primary care and mental health integration, and provided organizations with the opportunity to solicit feedback from the professional and clinic levels on what they need to make integration work as well as the opportunity to ensure that guidance addresses provider and clinic needs and concerns. However, while structural models can play an important role, it is not apparently sufficient as 
evidenced by the variation in the experiences of the three CCOs included in this study. Rather there is a need for leadership and guidance; stakeholders must actively utilize this tool as well as others to effectively drive efforts to integrate primary care and mental health.

In addition, Valentijn and his colleagues posit that normative enablers have an impact on the development of integration initiatives, while functional enablers impact the implementation of integration initiatives (2013). The findings of this study aligned with this supposition. Securing stakeholder commitment and buy-in is critical for the development of strategy. Without normative factors in place such as shared vision, trust, and visionary leadership, it is impossible to bring together stakeholders to consider the possibilities for integrating mental health and primary care. However, without functional factors such as resources, technology, and policies, meaningful integration is not likely to occur.

In terms of integration, the primary focus and the majority of accomplishments for all three CCOs have been centered on the integration of mental health services in primary care settings. Community mental health care continues to struggle with high turnover rates among providers and issues of access. Mental health providers have lower wages and higher administrative burdens compared to primary care providers. While there are larger system level issues at play that make it challenging to integrate primary care services into community mental health settings, and given the serious health disparities for individuals with SMI, it is vital that policymakers and other health care 
stakeholders actively pursue strategies and solutions to these issues so that communities can ensure that primary care services are available in community mental health settings.

It is also imperative that researchers develop a more nuanced understanding of integration and how to measure it (i.e., structures, processes, and outcomes). Models such as the RMIC, if refined and tested, hold great potential. Such models could be used to help create measures of integration processes which could then be combined with measures of expected integration outcomes to provide guidance on integrating primary care and mental health; this guidance may lead to more broad scale acceptance and adoption of effective primary care and mental health integration.

This study provided a broader understanding of the potential for organizations to facilitate integration of primary care and mental health. Overall findings confirm that integrating primary care and mental health is complex but that organizations can play an important role by ensuring the development of normative and functional enablers of integration at all levels of the system. The potential for organizations such as CCOs to facilitate integration of primary care and mental health is promising for addressing system fragmentation and improving health and health outcomes. 


\section{References}

Abernethy, M., \& Stoelwinder, J. (1995). The role of professional control in the management of complex organizations. Account Org Soc, 20(1), 1-17.

Anderson, R.A. \& McDaniel, R.R.Jr. (2000). Managing health care organizations: Where professionalism meets complexity science. Health Care Manage $R$, 25(1), 83-92.

Annamalai, A., Singh, N., \& Malley, S. S. O. (2015). Smoking use and cessation among people with serious mental illness. Yale J Biol Med, 88, 271-277.

Ansseau, M., Dierick, M., Buntinkx, F., Cnockaert, P., De Smedt, J., Van Den Haute, M., \& Vander Mijnsbrugge, D. (2004). High prevalence of mental disorders in primary care. J Affect Disord, 78(1), 49-55.

Autor, D., \& Duggan, M. (2006). The growth in the social security disability rolls: A fiscal crisis unfolding (Working Paper No. 12436). Retrieved from National Bureau of Economic Research website: http://www.nber.org/papers/w12436.

Axelsson, R., \& Axelsson, S. B. (2006). Integration and collaboration in public health--a conceptual framework. Int J Health Plan M, 21(1), 75-88.

Baillargeon, J., Thomas, C. R., \& Williams, B. (2008). Emergency department utilization patterns among uninsured patients with psychiatric disorders. Psychiatr Serv, 59, 808-811.

Bassuk, E. L., \& Gerson, S. (1978). Deinstitutionalization and mental health services. Sci Am, 238, 46-53.

Begun, J., Zimmerman, B., \& Dooley, K. (2003). Health care organizations as complex adaptive systems. In S. Mick \& M. Wyttenbach (Eds.), Advances in Health Care Organization Theory. San Francisco: Jossey-Bass.

Berwick, D. M., Nolan, T. W., \& Whittington, J. (2008). The Triple Aim: Care, health, and cost. Health Affairs, 27(3), 759-769.

Bhuvaneswar, C., Baldessarini, R., Harsh, V., \& Alpert, J. (2009). Adverse endocrine and metabolic effects of psychotropic drugs: Selective clinical review. CNS Drugs, 23(12), 1003-1021.

Birn, A.-E., Brown, T. M., Fee, E., \& Lear, W. J. (2003). Struggles for national health reform in the United States. Am J Public Health, 93(1), 86-91. 
Blount, A. (2003). Integrated primary care: Organizing the evidence. Fam Syst Health, 21, 121-134.

Blount, A., Schoenbaum, M., Kathol, R., Rollman, B. L., Thomas, M., O'Donohue, W., \& Peek, C. J. (2007). The economics of behavioral health services in medical settings: A summary of the evidence. Prof Psychol - Res Pr, 38(3), 290-297.

Bodenheimer, T. (1999). The Oregon Health Plan - Lessons for the nation. New Engl J Med, 337(9), 651-655.

Brady, K., \& Sinha, R. (2005). Co-occurring mental and substance use disorders: The neurological effects of chronic stress. Am J Psychiatry, 162(8), 14831493.

Butler, M., Kane, R.L., McAlpine D.D., Kathol, R.G., Fu S., Hagedorn H, \& Wilt T. (2008). Integration of mental health/substance abuse and primary care, AHRQ Publication No. 09-E0003. Rockville, MD: Agency for Healthcare Research and Quality.

Center for Behavioral Health Statistics and Quality. (2016). Key substance use and mental health indicators in the United States: Results from the 2015 National Survey on Drug Use and Health (HHS Publication No. SMA 164984, NSDUH Series H-51). Retrieved from http://www.samhsa.gov/data/

Centers for Medicare and Medicaid. (n.d.). Behavioral health services. Retrieved from http://www.medicaid.gov/medicaid-chip-program-information/bytopics/benefits/mental-health-services.html on February 17, 2015

Centers for Medicare and Medicaid. (2013). Application of the Mental Health Parity and Addiction Equity Act to Medicaid MCOs, CHIP, and Alternative Benefit (Benchmark) Plans. Retrieved from https://www.medicaid.gov/Federal-Policy-Guidance/downloads/SHO-13001.pdf on February 17, 2015

Centers for Medicare and Medicaid. (2015). Medicaid fact sheet: Mental health parity proposed rule for Medicaid and CHIP. Retrieved from http://www.medicaid.gov/medicaid-chip-program-information/bytopics/benefits/downloads/medicaid-fact-sheet-parity.pdf on February 17, 2015

Cohen, S. (1983). The mental hygiene movement, the development of personality and the school: The medicalization of American education. Hist Educ Quart, 23(2), 123-149. 
Cohen, S. B. (2014). The concentration of health care expenditures and related expenses for costly medical conditions, 2012. AHRQ Statistical Brief \#455. Rockville, MD: Agency for Healthcare Research and Quality.

Collins, C., Hewson, D. L., Munger, R., \& Wade, T. (2010). Evolving models of behavioral health integration in primary care. New York, NY: Milbank Memorial Fund.

Colton, C. W., \& Manderscheid, R. W. (2006). Congruencies in increased mortality rates, years of potential life lost, and causes of death among public mental health clients in eight states. Prev Chronic Dis, 3(2), A42.

Corrigan, P. (2004). How stigma interferes with mental health care. Am Psychol, 59(7), 614-625.

Creswell, J.W. \& Miller, D.L. (2000). Determining validity in qualitative inquiry. Theor Prac, 39(3): 124-130.

Croft, B., \& Parish, S. L. (2013). Care integration in the Patient Protection and Affordable Care Act: Implications for behavioral health. Admin Policy Ment HIth, 40(4), 258-63.

Croghan, T., \& Brown, J. (2010). Integrating mental health treatment into the patient centered medical home. AHRQ Publication No. 10-0084-EF. Rockville, MD: Agency for Healthcare Research and Quality.

Danziger, S., Frank, R., \& Meara, E. (2009). Mental illness, work, and income support programs. Am J Psychiatry, 166(4), 398-404.

Darawsheh, W. (2014). Reflexivity in research: Promoting rigour, reliability and validity in qualitative research. Int $J$ Ther Rehabil, 21(12), 560-568.

Davies, J., \& Janosik, E. (1991). Mental Health and Psychiatric Nursing. Burlington MA: Jones and Bartlett Learning.

Davis, M.M., Balasubramanian, B.A., Cifuentes, M.C., Hall, J., Gunn, R., Fernald, D., ... Cohen, D. (2015). Clinician staffing, scheduling, and engagement strategies among primary care practices delivering integrated care. J Am Board Fam Med, 28 (no. Supplement 1), S32-S40.

Dixon, K. (2009). Implementing mental health parity: the challenge for health plans. Health Affairs, 28(3), 663-665. 
Doherty, W. (1995). The Why's and Levels of Collaborative Family Health Care. Fam Syst Med, 13(3-4), 275-281.

Drake, R. E., Teague, G. B., \& Gersing, K. (2005). State Mental Health Authorities and Informatics. Community Ment Health J, 41(3), 365-370.

Droppers, O.J. (2014). A case study of collaborative governance: Oregon health reform and Coordinated Care Organizations (Doctoral dissertation). Paper 1824. Retrieved from http://pdxscholar.library.pdx.edu/open access etds/1824

Druss, B. (2007). Improving medical care for persons with serious mental illness: challenges and solutions. J Clin Psychiatry 2007; 68(suppl 4): 40-44.

Druss, B. G., \& Bornemann, T. H. (2010). Improving health and health care for persons with serious mental illness: The window for US federal policy change. JAMA, 303(19), 1972-1973.

Druss, B.G., \& Mauer, B.J. (2010). Healthcare reform and care at the behavioral health-primary care interface. Psychiatr Serv, 61(11), 1087-1092.

Druss, B. G., \& von Esenwein, S. A. (2006). Improving general medical care for persons with mental and addictive disorders: Systematic review. Gen Hosp Psychiatry, 28(2), 145-153.

Druss, B. G., \& Walker, E. R. (2011). Mental disorders and medical comorbidity. Research Synthesis Report No. 21. Princeton, NJ: Robert Wood Johnson Foundation.

Essock, S. M., Dowden, S., Constantine, N. T., Katz, L., Swartz, M. S., Meador, K. G., ... Rosenberg, S. D. (2003). Risk factors for HIV, hepatitis B, and hepatitis C among persons with severe mental illness. Psychiatr Serv, 54(6), 836-841.

Ferrante, J. M., Balasubramanian, B. a., Hudson, S. V., \& Crabtree, B. F. (2010). Principles of the patient-centered medical home and preventive services delivery. Ann Fam Med, 8(2), 108-116.

Fenton, M.C., Keyes, K., Geier, T., Greenstein, E., Skodol, A., Kruegar, B., . . . Hasin, D. (2012). Psychiatric comorbidity and the persistence of drug use disorders in the United States. Addiction, 107(3), 599-609.

Frank, R., \& Glied, S. (2008). Better but not well. Baltimore, Maryland: Johns Hopkins University Press. 
Friedman, L., \& Goes, J. (2001). Why integrated health networks have failed. Front Health Serv Manage, Summer 17(4), 3-28.

Fuchs, V. R., \& Emanuel, E. J. (2005). Health care reform: Why? What? When? Health Affairs, 24(6), 1399-1414.

Gelmon, S., Wallace, N., Sandberg, B., Petchel, S. \& Bouranis, N. (2016). Implementation of Oregon's PCPCH Program: Exemplary practice and program findings. Oregon Health Authority. Portland, OR; Oregon Health Authority.

Gerrity, M. (2014). Integrating primary care into behavioral health settings: What works for individuals with serious mental illness. New York, NY: Milbank Memorial Fund.

Gierisch, J., Nieuwsma, J., Bradford, D., Wilder, C., Mann-Wrobel, M., McBroom, A., ... Williams, J. W. (2013). Interventions to improve cardiovascular risk factors in people with serious mental illness. Comparative Effectiveness Reviews, No. 105. AHRQ Publication No. 13-EHC063-EF. Rockville, MD: Agency for Healthcare Research and Quality.

Gilfillan, R. J., Tomcavage, J., Rosenthal, M. B., Davis, D. E., Graham, J., Roy, J. A., ... Steele Jr., G. D. (2010). Value and the medical home: Effects of transformed primary care. Am J Manag Care, 16(8), 607-614.

Gioia, D., \& Chittipeddi, K. (1991). Sensemaking and sensegiving in strategic change initiation. Strateg Manage Jl, 12(6), 433-448.

Gladwell, M. (2002). The tipping point: How little things can make a big difference. Boston, MA: Back Bay Books.

Goldman, H. H., \& Grob, G. N. (2006). Defining "mental illness" in mental health policy. Health Affairs, 25(3), 737-749.

Goldman, H. H., \& Morrissey, J. P. (1985). The alchemy of mental health policy: Homelessness and the fourth cycle of reform. Am J Public Health, 75(7), 727-731.

Gostin, L. O. (2008). "Old" and "new" institutions for persons with mental illness: Treatment, punishment or preventive confinement? Public Health, 122(9), 906-913. 
Grant, B.F., Saha, T.D., \& Ruan, J. (2016). Epidemiology of DSM-5 drug use disorder: Results from the National Epidemiologic Survey on Alcohol and Related Conditions-III. JAMA Psychiatry, 73(1), 39-47

Gray, B. M., Weng, W., \& Holmboe, E. S. (2012). An assessment of patientbased and practice infrastructure-based measures of the patient-centered medical home: Do we need to ask the patient? BMC Health Serv Res, 47(1), 4-21.

Grob, G. N. (1992). Mental health policy in America: myths and realities. Health Affairs, 11(3), 7-22.

Grob, G. N. (1994). The mad among us: A history of the care of America's mentally ill. Washington, D.C.: Free Press.

Grumbach, K., \& Grundy, P. (2010). Outcomes of implementing patient centered medical home interventions: A review of the evidence from the prospective evaluation studies in the United States. Washington, D.C.: Patient Centered Primary Care Home Collaborative.

HB 3650, $76^{\text {th }}$ Oregon Legislative Assembly. (2011).

Hert, M. C., Correll, C. U., Cetkovich-Bakmas, M., Cohen, D., Asai, I., Detraux, J., ... Leucht, S. (2011). Physical illness in patients with severe mental disorders. World Psychiatry, 10, 52-77.

Horvitz-Lennon, M., Kilbourne, A. M., \& Pincus, H. A. (2006). From silos to bridges: Meeting the general health care needs of adults with severe mental illnesses. Health Affairs, 25(3), 659-669.

Hunter, G., Yoon, J., Blonigen, D., Asch, S., \& Zulman, D. (2015). Health care utilization patterns among high-cost $V A$ patients with mental health conditions. Psychiatr Serv, 66(9), 952-958.

Hwang, W., Chang, J., LaClair, M., \& Paz, H. (2013). Effects of integrated delivery system on cost and quality. Am J Manag Care, 19(5), e175-e184.

Institute of Medicine. (1997). Managing managed care: Quality improvement in behavioral health. Washington, D.C.: The National Academies Press.

Institute of Medicine. (2006). Improving the quality of health care for mental and substance-use conditions: Quality chasm series. Washington, D.C.: The National Academies Press. 
Jackson, G. L., Powers, B. J., Chatterjee, R., Prvu Bettger, J., Kemper, A. R., Hasselblad, V., ... Williams, J. W. (2013). The patient-centered medical home. Ann Intern Med, 158, 169-178.

James, J. (2015). Health policy brief: The Oregon health insurance experiment. Health Affairs.

Johnson, T., Sanders, D., \& Stange, J. (2014). The Affordable Care Act for behavioral health consumers and families. J Soc Work Disabil Rehabil, 13(12), 110-121.

Jones, D. R., Macias, C., Barreira, P. J., Fisher, W. H., Hargreaves, W., \& Harding, C. M. (2004). Prevalence, severity, and co-occurrence of chronic physical health problems of persons with serious mental illness. Psychiatr Serv, 55(11), 1250-1257.

Kahn, J. S., Aulakh, V., \& Bosworth, a. (2009). What it takes: Characteristics of the ideal personal health record. Health Affairs, 28(2), 369-376.

Kathol, R. G., Butler, M., McAlpine, D. D., \& Kane, R. L. (2010). Barriers to physical and mental condition integrated service delivery. Psychosom Med, 72(6), 511-518.

Kaye, N., Buxbaum, J., \& Takach, M. (2011). Building medical homes: Lessons from eight states with emerging programs. Commonwealth Fund pub. no. 1569. New York, NY: Commonwealth Fund.

Kessler, R., \& Chiu, W. (2005). Prevalence, severity, and comorbidity of twelvemonth DSM-IV disorders in the National Comorbidity Survey Replication (NCS- R). Arch Gen Psychiatry, 62(6), 617-627.

Knowles, P. (2009). Collaborative communication between psychologists and primary care providers. J Clin Psychol Med Settings, 16(1), 72-76.

Kuramoto, F. (2014). The Affordable Care Act and integrated care. J Soc Work Disabil Rehabil. 13(1-2):44-86.

Lagoe, R., Aspling, D. L., \& Westert, G. P. (2005). Current and future developments in managed care in the United States and implications for Europe. Health Res Policy Syst, 12(4), 1-12.

Lewis, V. A., Colla, C. H., Tierney, K., Van Citters, a. D., Fisher, E. S., \& Meara, E. (2014). Few ACOs pursue innovative models that integrate care for 
mental illness and substance abuse with primary care. Health Affairs, 33(10), 1808-1816.

Lim, R. F. (2012). Handbook of Community. New York: Springer New York.

Lopresti, A. L., \& Drummond, P. D. (2013). Obesity and psychiatric disorders: Commonalities in dysregulated biological pathways and their implications for treatment. Prog Neuropsychopharmacol Biol Psychiatry, 45, 92-99.

MacDowell, M., Glasser, M., Fitts, M., Nielsen, K., \& Hunsaker, M. (2010). A national view of rural health workforce issues in the USA. Rural Remote Health, 20(3), 1531-1547.

Maitlis, S. \& Christianson, M. (2014). Sensemaking in organizations: Taking stock and moving forward. Acad Manag Ann, 8(1), 57-125.

Markova, T., Mateo, M., \& Roth, L. M. (2012). Implementing teams in a patientcentered medical home residency practice: Lessons learned. J Am Board Fam Med, 25(2), 224-231.

Mauer, B. (2003). Behavioral health/primary care integration models, competencies, and infrastructure. Washington, D.C.: National Council for Community Behavioral Healthcare.

Mauer, B., \& Druss, B. (2010). Mind and body reunited: Improving care at the behavioral and primary healthcare interface. J Behav Health Serv Res, 37(4), 529-542.

Mays, N. \& Pope, C. (2000). Assessing quality in qualitative research. BMJ, 320, 50-52.

McConnell, K. J., Chang, A. M., Cohen, D. J., Wallace, N., Chernew, M. E., Kautz, G., ... Smith, J. (2014). Oregon's Medicaid transformation: An innovative approach to holding a health system accountable for spending growth. Healthc (Amst.), 2(3), 163-167.

McConnell, K.J. (2016). Oregon's Medicaid Coordinated Care Organizations. JAMA, 315(9), 869-870.

Mechanic, D. (2007). Mental health services then and now. Health Affairs 26(6), 1548-1550. 
Mechanic, D. (2012). Seizing opportunities under the Affordable Care Act for transforming the mental and behavioral health system. Health Affairs, 31(2), 376-382.

Mechanic, D. (2014). More people than ever before are receiving behavioral health care in the United States, but gaps and challenges remain. Health Affairs, 33(8), 1416-1424.

Mechanic, R. E., \& Altman, S. H. (2009). Payment reform options: Episode payment is a good place to start. Health Affairs, 28(2), 262-271.

Megna, J., Schwartz, T., Siddiqui, U., \& Herrera Rojas, M. (2011). Obesity in adults with serious and persistent mental illness: A review of postulated mechanisms and current interventions. Ann Clin Psychiatry, 23(2), 131-140.

Miles, MB. \& Huberman, AM. (1994). Qualitative Data Analysis (2nd edition). Thousand Oaks, CA: Sage Publications.

Miller, B. F., Mendenhall, T. J., \& Malik, A. D. (2009). Integrated primary care: An inclusive three-world view through process metrics and empirical discrimination. J Clin Psychol Med Settings, 16(1), 21-30.

Mintzberg, H. (1993). Structure in fives: Designing effective organizations. Englewood Cliffs, NJ: Prentice-Hall.

Mitchell, S. M., \& Shortell, S. M. (2000). The governance and management of effective community health partnerships: A typology for research, policy, and practice. The Milbank Quarterly, 78(2), 241-289.

Mittler, J., Gold, M., \& Lyons, B. (1999). Managed care and low-income populations: Four years' experience with the Oregon Health Plan. Washington, DC: Kaiser/Commonwealth Low-Income and Access Project.

National Conference of State Legislatures. (2015). State health insurance mandates and the ACA essential benefits provisions. Retrieved from http://www.ncsl.org/research/health/state-ins-mandates-and-aca-essentialbenefits.aspx on February 11, 2015

National Institute of Drug Abuse [NIDA]. (2017). Health Consequences of Drug Misuse. Retrieved from https://www.drugabuse.gov/related-topics/healthconsequences-drug-misuse on April 23, 2017

Norton, J., De Roquefeuil, G., Boulenger, J. P., Ritchie, K., Mann, A., \& Tylee, A. (2007). Use of the PRIME-MD Patient Health Questionnaire for estimating 
the prevalence of psychiatric disorders in French primary care: A comparison with family practitioner estimates and relationship to psychotropic medication use. Gen Hosp Psychiatry, 29(4), 285-293.

Novella, E. (2010). Mental health care in the aftermath of deinstitutionalization: A retrospective and prospective view. Health Care Anal, 18(3), 222-238.

Office for Oregon Health Policy and Research. (2010). Standards and measures for patient centered primary care homes. Portland, OR. Retrieved from http://www.oregon.gov/oha/action-plan/pcpch-report.pdf on February 17, 2015

Oregon Office for Rural Health. (n.d.) Rural definitions. Retrieved from http://www.ohsu.edu/xd/outreach/oregon-rural-health/data/rural-definitions/ on February 11, 2017

Oregon Health Authority. (n.d.a.). Coordinated care: The Oregon difference. Retrieved from http://www.oregon.gov/oha/OHPB/pages/healthreform/ccos.aspx on November 16, 2015

Oregon Health Authority. (n.d.b). Certified Community Behavioral Health Clinics: $\mathrm{CCBHC}$ demonstration program. Retrieved from http://www.oregon.gov/oha/bhp/Pages/Community-BH-Clinics.aspx on April 17, 2017.

Oregon Health Authority. (n.d.c). Metrics and Scoring Committee. Retrieved from http://www.oregon.gov/oha/analytics/Pages/Metrics-ScoringCommittee.aspx on March 21, 2015.

Oregon Health Authority. (n.d.d.). Oregon Coordinated Care Organizations transformation plans. Retrieved from http://www.oregon.gov/oha/OHPB/Pages/health-reform/certification/OregonCCO-Transformation-Plans.aspx on March 21, 2015

Oregon Health Authority. (2010). The future of health and human services in Oregon. [Pamphlet]. Salem, Oregon.

Oregon Health Authority. (2016). Behavioral Health Collaborative Report. Retrieved from https://www.oregon.gov/oha/bhp/Documents/BehavioralHealth-Collaborative-Report.pdf on April 12, 2017.

Oregon Health Policy Board. (2012). Coordinated Care Organizations implementation proposal. Salem, OR: Oregon Health Authority. 
Osborn, D., Levy, G., Nazareth, I., Peterson, I., Islam, A., \& King, M. B. (2007). Relative risk of cardiovascular and cancer mortality in people with severe mental illness from the United Kingdom's general research database. Arch Gen Psychiatry, 64, 242-249.

Owens, P.L., Mutter, R., \& Stocks, C. (2010). Mental health and substance abuse-related emergency visits among adults, 2007. HCUP Statistical Brief \#92. July 2010. Agency for Healthcare Research and Quality, Rockville, MD. http://www.hcup-us.ahrq.gov/reports/statbriefs/sb92.pdf

Peikes, D., Zutshi, A., Genevro, J., Smith, K., Parchman, M., \& Meyers, D. (2012). Early evidence on the patient-centered medical home. Rockville, MD: Agency for Healthcare Research and Quality.

Pendlebury, J., \& Holt, R. I. G. (2010). Managing diabetes in people with severe mental illness, J Diabetes Nurs, 14(9): 328-339.

Pincus, H. A. (2003). The future of behavioral and primary care: Drowning in the mainstream or left on the banks? Psychosomatics, 44(1): 1-11.

Pitts, S. R., Carrier, E. R., Rich, E. C., \& Kellermann, a. L. (2010). Where Americans get acute care: Increasingly, it's not at their doctor's office. Health Affairs, 29(9), 1620-1629.

Plsek, P. E., \& Greenhalgh, T. (2001). The challenge of complexity in health care. BMJ, 323, 625-628.

Randall, D., \& Parente, S. (2012). U. S. Medicaid managed care markets: Explaining state policy choice variation. Insurance Markets and Companies: Analyses and Actuarial Computations, 3(1), 35-49.

Regier, D. A., Goldberg, I. D., \& Taube, C. A. (1978). The de facto US mental health services system: A public health perspective. Arch Gen Psychiatry, 35(6), 685-693.

Reiss-Brennan, B., Briot, P.C., Savitz, L.A., Cannon, W. \& Staheli, R. (2010). Cost and quality impact of Intermountain's mental health integration program. J Healthc Manag, 55(2), 97-113.

Review, I. N. (2010). Geisinger Medical Home Pilot Demonstrates Success. Communications, 17(12), 545-546.

Rissi, J., Gelmon, S., Saulino, E., Merrithew, N., Baker, R., \& Hatcher, P. (2014). Building the foundation for health system transformation: Oregon's Patient- 
Centered Primary Care Home program. J Public Health Manag Pract, 21(1), $34-41$.

Rittenhouse, D. R., Schmidt, L., Wu, K., \& Wiley, J. (2013). Contrasting trajectories of change in primary care clinics: Lessons from New Orleans safety net. Ann Fam Med, 11(Suppl. 1), 60-67.

Robson, D., \& Gray, R. (2007). Serious mental illness and physical health problems: A discussion paper. Int J Nurs Stud, 44, 457-466.

Rogers, E., Medina, U., Rivera, M., \& Wiley, C. (2005). Complex adaptive systems and the diffusion of innovations. The Innovations Journal: The Public Sector Innovation Journal, 11(3), 1-26.

Rosenthal, M. (2010). Beyond pay for performance - Emerging models of provider-payment reform. New Engl J Med, 363(1), 1-3.

Rosenthal, T. C. (2008). The medical home: Growing evidence to support a new approach to primary care. J Am Board Fam Med, 21(5), 427-440.

Rüsch, N., Angermeyer, M. C., \& Corrigan, P. W. (2005). Mental illness stigma: Concepts, consequences, and initiatives to reduce stigma. Eur Psychiatry, 20(8), 529-539.

Saloner, B., \& Le Cook, B. (2014). An ACA provision increased treatment for young adults with possible mental illnesses relative to comparison group. Health Affairs, 33(8), 1425-1434.

Saloner, B., Polsky, D., Kenney, G. M., Hempstead, K., \& Rhodes, K. V. (2015). Most uninsured adults could schedule primary care appointments before the ACA, but average price was $\$ 160$. Health Affairs, 34(5), 773-780.

Salsberry, P., Chipps, E., \& Kennedy, C. (2005). Use of general medical services among Medicaid patients with severe and persistent mental illness. Psychiatr Serv, 56(4), 458-462.

Savage, G., Taylor, R., Rotarius, T., \& Buesseler, J. (1997). Governance of integrated delivery systems/networks: A stakeholder approach. Health Care Manage Rev., 22(1), 7-20.

Scott, R. (2003). Organizations: Rational, natural, and open systems. Upper Saddle River, NJ: Prentice-Hall. 
Senn, T. E., \& Carey, M. P. (2009). HIV testing among individuals with a severe mental illness: Review, suggestions for research, and clinical implications. Psychol Med, 39(3), 355-63.

Sharfstein, S. S. (2000). Whatever happened to community mental health? Psychiatr Serv, 51(5), 616-620.

Shen, C., Sambamoorthi, U., \& Rust, G. (2008). Co-occurring mental illness and health care utilization and expenditures in adults with obesity and physical illness. Dis Manag, 11(3), 153-160.

Shih, A., Davis, K., Schoenbaum, S. C., Gauthier, A., Nuzum, R., \& McCarthy, D. (2008). Organizing the U.S. health care delivery system for high performance. New York, NY: The Commonwealth Fund.

Shortell, S. M., \& Mccurdy, R. K. (2010). Integrated health systems. Stud Health Technol Inform, no. 153: 369-82.

Smith, W.K. \& Lewis, M.W. (2012). Leadership skills for managing paradoxes. Ind Organ Psychol, 5(2), 227-231.

Sparer, M. (2012). Medicaid managed care: Costs, access, and quality of care. Research Synthesis Report No. 23. Princeton, NJ: Robert Wood Johnson Foundation.

Spitzer, R. L., lii, F. V, Hahn, S. R., Linzer, M., Williams, J. B. W., Brody, D., \& Davies, M. (1997). Multisomatoform disorder: An alternative to undifferentiated somatoform disorder for the somatizing patient in primary care. Arch Gen Psychiatry, 54, 352-358.

Sprague, L. (2003). Disease management to population-based health: Steps in the right direction? NHPF Issue Brief No. 791. Washington, D.C.: National Heath Policy Forum.

Starfield, B. (2011). Challenges to primary care from co- and multi-morbidity. Prim Health Care Res Dev, 12(1), 1-2.

Starfield, B., Shi, L., \& Macinko, J. (2005). Contribution of primary care to health systems and health. The Milbank Quarterly, 83 (3), 457-502.

Stenger, R. J., Smith, J., Mcmullan, J. B., Rodriguez, G. S., Dorr, D. A., Minniti, M., ... Anderson, M. (2012). Defining the medical home: The Oregon experience. J Am Board Fam Med, 25, 869-877. 
Strozier, M., \& Walsh, M. (1998). Developmental models for integrating medical and mental healthcare. Fam Syst Health, 16(1-2), 27-40.

Sturm, R., Mcculloch, J., Garcia, R. a, Act, A., Code, I. R., Forces, A., ... Sarata, A. K. (2011). Moving beyond parity - Mental health and addiction care under the ACA. New Engl J Med, 31(11), 973-975.

Substance Abuse and Mental Health Administration. (n.d.). Health care and health systems integration. Retrieved from http://www.samhsa.gov/healthcare-health-systems-integration on February 21, 2015

Substance Abuse and Mental Health Services Administration. (2012). Behavioral health homes for people with mental health \& substance use conditions: The core clinical features. Washington, D.C.: SAMHSA.

Suter, E., Oelke, N. D., Adair, C. E., \& Armitage, G. D. (2009). Ten key principles for successful health systems integration. Healthcare Quarterly, 13 (Spec No), 16-23.

Swendsen, J., Conway, K.P., Degenhardt, L., Glantz, M., Jin, R., Merikangas, K.R., ... Kessler, R.C. (2010). Mental disorders as risk factors for substance use, abuse and dependence: results from the 10-year follow-up of the National Comorbidity Survey. Addiction, 105(6), 1117-1128.

Takach, M. (2011). Reinventing Medicaid: State innovations to qualify and pay for patient-centered medical homes show promising results. Health Affairs, 30(7), 1325-1334.

Taylor, F. (2008). The principles of scientific management. Overland Park, KS: Digireads.com. (Original work published 1911)

Toalson, P., Ahmed, S., Hardy, T., \& Kabinoff, G. (2004). The metabolic syndrome in patients with severe mental illnesses. Prim Care Companion $J$ Clin Psychiatry, 6(4), 152-158.

Torrey, E. F. (1997). Out of the shadows: Confronting America's mental illness crisis. New York, NY: John Wiley \& Sons, Inc.

Trubek, L. G. (2006). New governance and soft law in health care reform. Ind Health Law Rev, 3(137).

Tuohy, C. H. (2003). Agency, contract and governance: Shifting shapes of accountability in the health care arena. J Health Polit Policy Law, 28(2-3), 195-215. 
U.S. Department of Health and Human Services. (1999). Mental health: A report of the Surgeon General. Rockville, MD: U.S. Department of Health and Human Services, Substance Abuse and Mental Health Services Administration, Center for Mental Health Services, National Institutes of Health, National Institute of Mental Health.

U.S. Department of Health and Human Services. (2016). HHS selects Oregon for a new program to improve access to high quality behavioral health services. The Lund Report. Portland, OR. Retrieved from https://www.thelundreport.org/content/hhs-selects-oregon-new-programimprove-access-high-quality-behavioral-health-services on April 17, 2017.

Valentijn, P. P., Boesveld, I. C., van der Klauw, D. M., Ruwaard, D., Struijs, J. N., Molema, J. J. W., . . Vrijhoef, H. J. (2015). Towards a taxonomy for integrated care: A mixed-methods study. Int J Integr Care, 15, 1-18.

Valentijn, P. P., Schepman, S. M., Opheij, W., \& Bruijnzeels, M. A. (2013). Understanding integrated care: A comprehensive conceptual framework based on the integrative functions of primary care. Int $J$ Integr Care, 13, 112.

Valentijn, P. P., Vrijhoef, H. J. M., Ruwaard, D., Boesveld, I., Arends, R. Y., \& Bruijnzeels, M. A. (2015). Towards an international taxonomy of integrated primary care: A Delphi consensus approach. BMC Fam Pract, 16(1), 64-81.

Vourlekis, B. S. (2011). Social work and aftercare of the mentally ill in Maryland: The early years. Retrieved from http://www.socialwelfarehistory.com/programs/social-work-and-aftercare-ofthe-mentally-ill-in-maryland/ on February 11, 2015

Wagner, E. H., Coleman, K., Reid, R. J., Phillips, K., \& Sugarman, J. (2012). Guiding transformation: How medical practices can become patient-centered medical homes. New York, NY: The Commonwealth Fund.

Waxmonsky, J., Auxier, A., Heath, B., \& Wise Romero, P. (2014). Integrated practice assessment tool. Washington, DC: SAMHSAHRSA Center for Integrated Health Solutions. Retrieved from http://www.integration.samhsa.gov/operationsadministration/IPAT v 2.0 FINAL.pdf on July 17, 2016.

Weber, E. (2013). Equality standards for health insurance coverage: Will the Mental Health Parity and Addiction Equity Act end the discrimination? Golden Gate U L Rev, 43(2), 179-259. 
Weick K, Sutcliffe K. 2007. Managing the unexpected: Resilient performance in an age of uncertainty. San Francisco, CA: Jossey Bass.

Weiss, A.J., Barrett, M.L., Heslin, K.C., \& Stocks, C. (2016). Trends in emergency department visits involving mental and substance use disorders, 2006-2011. Statistical Brief \#216. Healthcare Cost and Utilization Project (HCUP). December 2016. Agency for Healthcare Research and Quality, Rockville, MD.

Whiteford, H.A., Degenhardt, L., Rehm, J., Baxter, A.J., Ferrari, A.J., Erskine, H.E., ... Vos, T. (2013). Global burden of disease attributable to mental and substance use disorders: findings from the Global Burden of Disease Study 2010. The Lancet, 382(9904), 1575-1586.

WHO-Wonca. (2008). Integrating mental health and primary care: A global perspective. (M. Funk \& G. Ivbijaro, Eds.). Geneva, Switzerland: World Health Organization Press.

Wynia, M. K., VanGeest, J. B., Cummins, D. S., \& Wilson, I. B. (2003). Do physicians not offer useful services because of coverage restrictions? Health Affairs, 22(4), 190-197.

Wu, L. \& Blazer, D.G. (2014). Substance use disorders and psychiatric comorbidity in mid and later life: A review. Int J Epidemiol, 43(2), 304-317.

Yin, R. K. (2013). Case study research: Design and methods (5 $5^{\text {th }}$ ed.). New York, NY: Sage Publications. 


\section{Appendix A: CCO Interview Protocol and Consent Narrative}

"My name is Robin Baker, and I am a doctoral student in Health Systems and Policy program at Portland State University. As part of the requirements of the doctoral program, I am conducting a research project studying Oregon's Coordinated Care Organizations (CCOs). As the [position of research participant] for the [CCO name], you have been identified as knowledgeable about primary care and mental health integration at [CCO name]. As all CCOs are tasked with integrating care, this is an opportunity to learn about conditions that facilitate or impede the ability to effectively integrate primary care and mental health and develop insights into similarities and differences across CCOs.

The purpose of this interview is to capture your perspectives about the early development and implementation of your CCOs strategy for integrating primary care and mental health, and to identify factors that facilitate or impede achievement of that strategy from your professional perspective.

The interview will last approximately one hour. With your permission, I will take notes and record the interview. Your participation in this interview is voluntary; you do not have to answer any question that you do not want to answer and you may stop the interview at any time. All individual responses will be kept confidential. Given four of 16 of the CCOs have been included in this project, it may be somewhat difficult to completely blind the organization that is the source of the comments, but I will de-identify individual respondents.

The recording and my notes will help me to accurately represent our discussion; no one else will ever hear the recordings or see the written transcripts. If there are things that you tell me that you do not wish repeated, please indicate this so that I do not include those comments in any summaries or reports that I develop from this interview. Similarly, if at any time you would like me to stop recording, please indicate this and I will turn off the recorder. Findings will be reported in the aggregate with larger themes identified within and across the CCOs. Quotes will be selected to illustrate these broader themes and will be presented without attribution to individuals. At this time, do you have any questions or concerns?

[After addressing any questions and/or concerns state...]

Then let us begin." 
Date:

ID No:

1. I'd like to start with asking you to briefly describe [CCO name] and your role at [CCC name].

- What is your involvement in efforts to facilitate primary care and mental integration?

2. What is [CCO name]'s strategy for the integration of primary care and mental health services?

- Who is the target population for [CCO name]'s strategy for integrated primary care and mental health services?

3. Please talk about the process for developing the integration strategy.

- What led to [CCO name] choosing this particular strategy??

- Has that evolved? If so, how?

- Who represented the mental health care perspective?

- Who represented the primary care perspective?

- Were there any other interests included?

4. What provider organizations are involved in the strategy to integrate primary care and mental health?

- Please describe those relationships.

- Are they different from how they were in the past?

- What mechanisms are utilized to develop and maintain those partnerships? (e.g., inter-organizational agreements, payment reform)

5. How does the CCO strategy intend to change the way patients receive services in order to improve or achieve integration?

6. What role, if any, does [CCO name] play in ensuring that the specific elements of your integration strategy are in place or occur?

- What is the state doing that helps or hinders your efforts to facilitate integration?

- In what ways have state-level policies impacted [CCO name]'s efforts to facilitate integration? 
7. What have been the biggest barriers to managing the integration of primary care and mental health?

- How does [CCO name] manage these barriers?

8. What have been the biggest factors that have facilitated the integration of primary care and mental health?

9. What are some of the biggest accomplishments [CCO name] has accomplished in terms of integrating primary care and mental health?

- Are there any stories that exemplify [CCO name's] role in facilitating integration that you are willing to share?

10. What would you change about the environment to make integration more feasible?

- In the perfect world if the environmental conditions were already aligned to support integration, what else would [CCO name] do to facilitate the integration of primary care and mental health?

Closing: "Thank you for participating in this interview, and for your thoughtful comments, insights and candor. I am meeting with 5-7 other stakeholders from your $\mathrm{CCO}$ as well as from three other CCOs. I will be analyzing and synthesizing the key themes and issues that emerge over the course of the study. If you think of anything else, please contact me. Findings from the interviews will be included in my dissertation and read by PSU faculty serving on my dissertation committee. At the conclusion of the study, I would be happy to share a report of the aggregated findings with you. Again, I thank you for your time and willingness to participate." 


\section{Appendix B: Initial Email for Research Participant Recruitment}

Dear [CCO affiliated stakeholder]:

You are invited to participate in an interview as part of a research study I am conducting as a PhD candidate in the Health Systems and Policy PhD program at Portland State University under the supervision of Dr. Neal Wallace, Professor of Public Health. The purpose of the study is to explore the key factors that facilitate or impede the ability of Coordinated Care Organizations (CCOs) to encourage and support the integration of primary care and mental health. I am doing a multi-site case study with four CCOs across the state.

CCOs are leading the effort to integrate health for the purposes of achieving the Triple Aim. There is a unique opportunity to learn from [CCO name] about the challenges and opportunities in integrating primary care and mental health that cannot only help inform other CCOs but also guide the development of best practices in integration.

I will be conducting interviews with 6-8 executive leaders, senior staff, and other influential CCO stakeholders from four CCOs in the next few months. Each interview will be individual and last approximately 1 hour. Interviews will be faceto-face when possible; I will travel to a location of your convenience. The option of telephone or Skype interviews are also available for consideration.

I have attached the consent form that provides more detail regarding the study, your rights as a research participant, and any potential risks of participation as well as the steps I will take to mitigate them. At this time, I do not need you to sign the consent form. It is provided here to give you more information regarding the study.

If you have any concerns about your participation in this study or your rights as a research subject, you may contact the Human Subjects Research Review Committee, Office of Research and Strategic Partnerships, Portland State University, 620 Market Center Building, 1600 SW 4th Avenue, Portland, OR, USA 97201 or by telephone at $503-725-4288$ or $1-877-480-4400$. You may also email the office at hsrrc@lists.pdx.edu.

If you have any concerns about the research study, you may contact Dr. Wallace at 503-725-8248 or nwallace@pdx.edu, or me directly at xxx-xxx-xxxx or rlbaker@pdx.edu.

Your input is invaluable and will help me to gather important insights about CCO efforts to integrate primary care and mental health. If you agree to participate, please respond indicating your willingness to participate, and I will be in touch with you to schedule the interview. Thank you in advance for your consideration.

Robin Baker 


\section{Appendix C: Consent Form}

[On OHSU-PSU School of Public Health letterhead]

\section{Coordinated Care Organizations: Primary Care \& Mental Health Integration} Interview Consent Form

\section{Introduction and Purpose}

You are invited to participate in an interview as part of a research study Robin Baker is conducting as a PhD candidate in the Health Systems and Policy Program in the Mark O. Hatfield School of Government at Portland State University (PSU) under the supervision of Dr. Neal Wallace, Professor of Public Administration. The purpose of the study is to explore Oregon's coordinated care organizations (CCOs) in an effort to understand the impact of health reform on mental health services and to identify the factors that impede or facilitate the integration of primary care and mental health.

CCOs are leading the effort to transform Oregon's health care delivery system around the Triple Aim of improving health, health care, and lowering costs. Engaged in facilitating the integration primary care and mental health, Oregon's CCOs offer a unique opportunity to learn about the challenges and opportunities for integrating primary care and mental health. This information can help inform other CCOs and health care organizations and guide the development of best practices related to approaches to integrating mental health and primary care. If you decide to participate in the interview, you will be asked questions about your CCO's strategy for facilitating the integration of primary care and mental health and important factors that contributed to or impeded integration. There are no right or wrong answers to the questions; the important thing is for you to share your experience and opinions. However, please only share personal information that you feel comfortable discussing. You may not receive any direct benefit from taking part in this study aside from reflecting on your experiences, but the study may help increase knowledge which may help others in the future.

\section{Study Procedures}

Your participation will consist of an individual interview. Individual interviews will involve speaking one-on-one with Robin Baker. With your permission, the interview will be recorded and recordings will be transcribed into a secure database. None of your identifying information will be used in the database. The database will help Robin Baker analyze the discussions, identify themes from your $\mathrm{CCO}$ and across the other selected CCOs, and in particular help identify the key factors that impact the ability of CCOs to integrate care. You may choose not to participate in the future or end participation in the study at any time. 


\section{Possible Risks \& Benefits}

Some of the questions asked during the interview may be sensitive and make you feel uncomfortable. You can refuse to answer any question(s) asked during the interview or focus group.

In addition, any information that is obtained in connection with this study and that can be linked to you or identify you will be kept confidential. When the recording is transcribed, your name will not be used; instead your study ID will be utilized. The key that links your study ID to your name and transcriptions will be kept in a password protected datafile in a Dropbox folder; access to this Dropbox will only be available to Robin Baker. Recordings will be destroyed after transcription. Any data that could identify you (e.g., transcriptions and key) will be destroyed by Robin Baker after a period of no longer than three years.

The study records are private and only Robin Baker has the right to look at the records. It is important, however, that the Portland State University Institutional Review Board (IRB - a committee that reviewed this research to protect your rights) be able to look at the study records. Your specific responses will be kept confidential. The notes and recordings will help Robin Baker to accurately document the discussion, but no one else will hear the recordings or see the written transcripts.

If any of your interview comments are published in journals or reported at meetings, you will not be identified by name.

\section{General Information}

Your participation is voluntary. You do not have to take part in this study, and it will not affect your relationships with Portland State University or Oregon Health and Science University. You can choose not to answer any question, and you can leave the interview at any time.

You do not give up any of your legal rights by signing this consent form and taking part in this study.

If you have any concerns about your participation in this study or your rights as a research subject, you may contact the Human Subjects Research Review Committee, Office of Research and Strategic Partnerships, Portland State University, 620 Market Center Building, 1600 SW 4th Avenue, Portland, OR, USA 97201 or by telephone at 503-725-4288 or 1-877-480-4400. You may also email the office at hsrrc@lists.pdx.edu. If you have any concerns about the 
research study, you may contact Dr. Wallace at 503-725.8248 or nwallace@pdx.edu, or me directly at 503-810-8520 or rlbaker@pdx.edu.

Name of Participant (Please Print)

Signature of Participant Date INVESTIGATOR SIGNATURE

This research study has been explained to the participant and all of his/her questions have been answered. The participant understands the information described in this consent form and freely consents to participate.

Robin Baker

Name of Investigator

Signature of Investigator)

Date 